\title{
The Role of Hiwi in Stem Cell Maintenance and Sarcomagenesis
}

\author{
Sara Siddiqi
}

\author{
Submitted in partial fulfillment of the \\ requirements for the degree of \\ Doctor of Philosophy \\ under the Executive Committee \\ of the Graduate School of Arts and Sciences
}

Columbia University

2012 
(c) 2012

Sara Siddiqi

All rights reserved 


\title{
ABSTRACT \\ The Role of Hiwi in Stem Cell Maintenance and in Sarcomagenesis
}

\author{
Sara Siddiqi
}

Sarcomas are cancers of connective tissues, such as bone, adipose and cartilage, and are thought to arise from the aberrant development of the mesenchyme. As such, mesenchymal stem cells are thought to be the cell of origin for sarcomas. Genetic or epigenetic lesions at particular points during the differentiation of a mesenchymal stem cell into its terminal mesenchymal cell type are able to give rise to specific subtypes of sarcomas.

Recently, a number of reports have identified elevated expression of the human Piwi homolog—called Hiwi-in a variety of human cancers, including gastric cancer, pancreatic cancer, gliomas and, most relevant for this dissertation, sarcomas. In sarcomas, Hiwi is highly expressed and elevated Hiwi prognosticates shorter patient survival.

Hiwi is the human homolog of the Piwi family of proteins, which are members of the PazPiwi Doman (PPD) family. During normal development, Piwis are thought to maintain stem cells of the germline, and indeed their expression is limited to early development and to the adult germline. Piwis are thought to maintain stem cells in the germline with small RNA partners, called piwi-interacting RNAs (piRNAs). More specifically, Piwi/piRNA complexes in the germline are thought to maintain transposon silencing, and thus ensure genomic stability. A detailed mechanism by with Piwis suppress transposon migration in the germline remains an area of active investigation, but is thought to occur via DNA methylation of transposon regions. In this way, Piwis are critical for maintenance of genomic integrity of germline stem cells during normal development. Thus, the finding that Piwis are elevated in human cancers is directly in conflict with its known role in ensuring genomic stability during development. 
Piwi homologs are critical for maintenance of germline stem cells during development but aberrant Hiwi expression has also been identified in all cancers examined, including in sarcomas. A potential connection between mesenchymal stem cells, sarcomas and Hiwi remains unexplored. Moreover, the role of Hiwi in sarcomas is unknown.

In the studies presented here, we demonstrate that over-expressing Hiwi in mesenchymal stem cells inhibits their differentiation in vitro and generates sarcomas in vivo. Secondly, transgenic mice expressing Hiwi (mesodermally-restricted) develop sarcomas. Conversely, inducible down-regulation of Hiwi in human sarcomas inhibits growth and reestablishes differentiation. These data reveal that Hiwi is directly tumorigenic. We have also identified the presence of piRNAs in our Hiwi-expressing models. We further show that DNA methylation correlates with Hiwi expression and that cyclin-dependent kinase inhibitor (CDKI) tumor suppressor genes are silenced upon Hiwi over-expression. Moreover, Hiwi's tumorigenic effects are reversible using DNA de-methylating agents.

These studies reveal for the first time not only a novel oncogenic role for Hiwi as a driver of tumorigenesis, but also suggest that the use of epigenetic agents may be clinically beneficial for treatment of tumors that express Hiwi. Additionally, our data showing that Hiwi-associated DNA hyper-methylation with subsequent genetic and epigenetic changes favoring a tumorigenic state reconciles the conundrum of how Hiwi may act appropriately to promote genomic integrity during early development (via transposon silencing) and inappropriately in adult tissues with subsequent tumorigenesis. 


\section{Table of Contents}

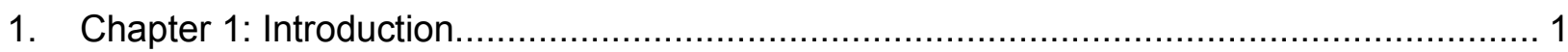

Sarcomas and mesenchymal stem cells ..................................................................... 1

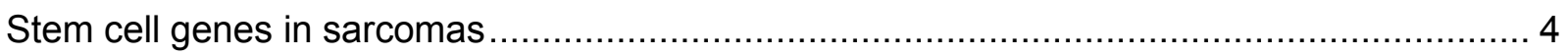

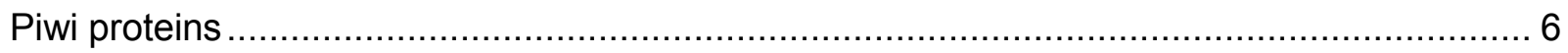

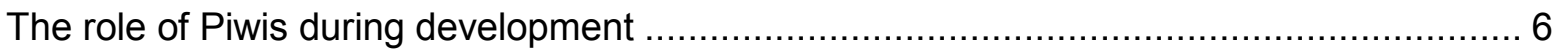

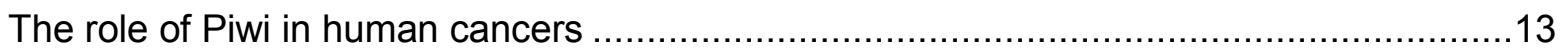

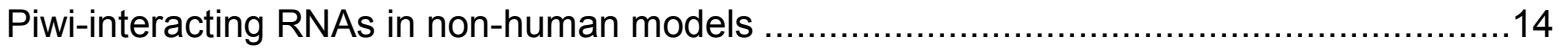

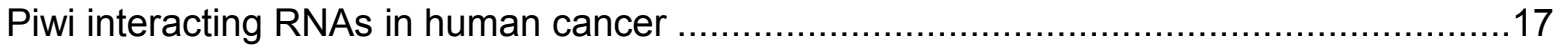

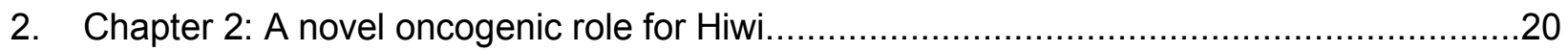

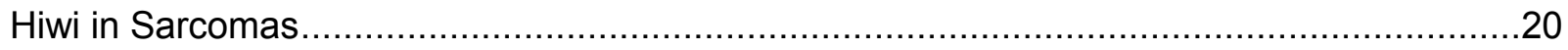

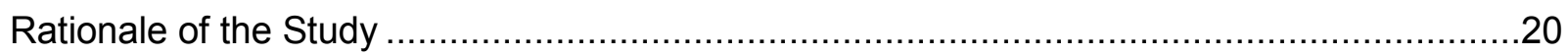

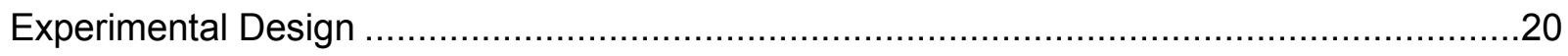

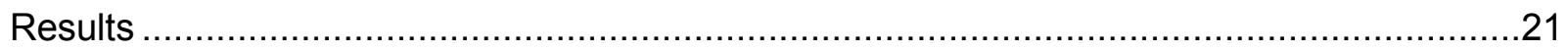

1. Hiwi is highly expressed in human primary undifferentiated sarcomas ......................21

2. Hiwi is not chromosomally amplified in Hiwi-expressing sarcomas...........................22

3. Generation of Hiwi over-expressing mesenchymal stem cells ................................24

4. Hiwi over-expression blocks mesenchymal differentiation capacity in vitro ................26

5. Hiwi over-expression transforms mesenchymal stem cells in vivo...........................28

6. Stem cells are more sensitive to Hiwi-mediated transformation................................30 
7. A mesoderm-specific transgenic mouse model of Hiwi develops lipomatous lesions ..32

8. Generation of short-hairpin Hiwi knock down sarcoma cells.

9. Short-hairpin Hiwi knock down re-establishes differentiation capacity and reduces

colony formation capacity.

Discussion

Materials and Methods

3. Chapter 3: Identification of piwi-interacting RNAs (piRNAs) in mesenchymal stem cells ....45 piRNAs are present in germline and somatic cells

Rationale of the Study

Experimental Design

Results

1. Small RNA sequencing reveals presence of piRNAs in mesenchymal stem cells

2. piRNA expression patterns do not shift in Hiwi-expressing cells.

3. piRNAs in mesenchymal stem cells do not correlate to gene sites.....

4. piRNAs in mesenchymal stem cells do not generally lie upstream of gene sites

Discussion

Materials and Methods

4. Chapter 4: Characterization of Hiwi-dependent epigenetic mechanism of tumorigenesis ...58

Piwis and DNA methylation .58

Rationale of the Study .58

Experimental Design .59 
Results

1. Transposon expression is decreased in Hiwi-expressing MSCs.

2. Global DNA methylation correlates directly with Hiwi expression level 60

3. DNA methyltransferase proteins are elevated in Hiwi-expressing MSCs 62

4. 5-azacytidine treatment restores transposon expression in Hiwi-expressing MSCs ...63

5. 5-azacytidine treatment reduces colony forming capacity of sarcoma cells . 66

6. Gene expression changes during 5-azacytidine treatment and during Hiwi knock down are very similar .67

7. Promoter methylation is unchanged by Hiwi expression. 69

8. Identification of methylation-dependent tumor suppressors via gene expression profiling .73

9. Cyclin dependent kinase inhibitor expression is altered in Hiwi-expressing cells ........77

10. Histone methylation marks remain unchanged during Hiwi knock down. 79

11. Determining methylated loci via STAMP assay .81

Discussion 82

Materials and Methods .86

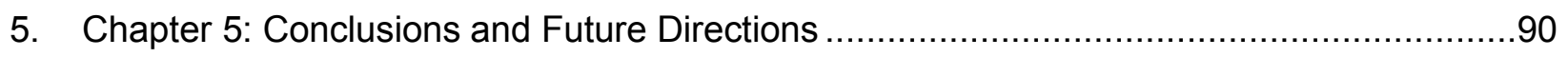

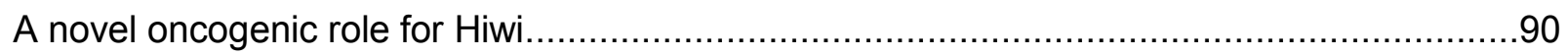

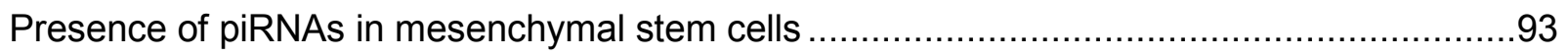

Hiwi-mediated tumorigenesis is associated with DNA methylation.................................99

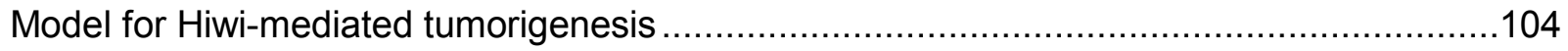




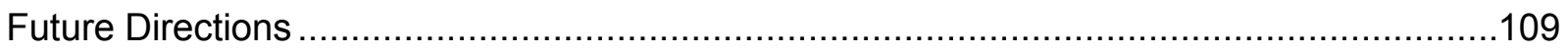

The next generation of transgenic mouse models of Hiwi over-expression ...................109

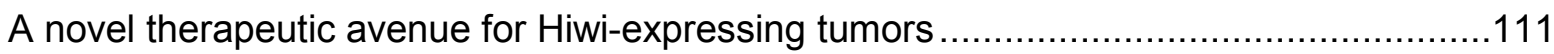

Potential global implications of our studies ...........................................................112

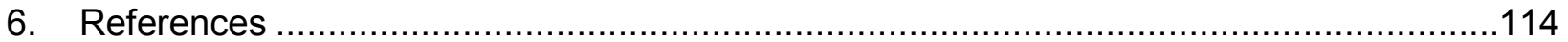




\section{List of Figures, Charts and Illustrations}

Figure 1.1 Differentiating MSCs transform to develop specific sarcoma subtypes........................ 3

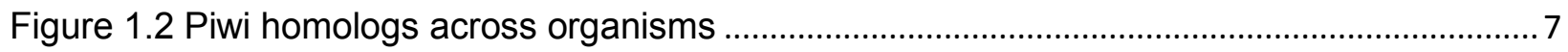

Figure 1.3 The PPD family is divided into the Ago and Piwi subfamilies .................................... 9

Figure 1.4 Ping-pong cycle of piRNA biogenesis .................................................................. 12

Figure 2.1 Hiwi is highly expressed in human undifferentiated sarcoma samples ......................22

Figure 2.2 Hiwi is not chromosomally amplified in Hiwi-expressing sarcomas .............................24

Figure 2.3 Generation of Hiwi-expressing mesenchymal stem cells ........................................26

Figure 2.4 Hiwi over-expression blocks mesenchymal differentiation in vitro .............................28

Figure 2.5 Hiwi transforms MSC to become tumorigenic in vivo .................................................. 30

Figure 2.6 Stem cells are more sensitive to Hiwi-mediated transformation ..................................32

Figure 2.7 Mesoderm-specific transgenic mouse model of Hiwi over-expression .........................33

Figure 2.8 Generation of short-hairpin Hiwi knock down sarcoma cells....................................... 35

Figure 2.9 Hiwi knock down re-establishes differentiation and reduces colony formation of

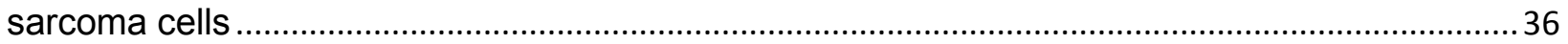

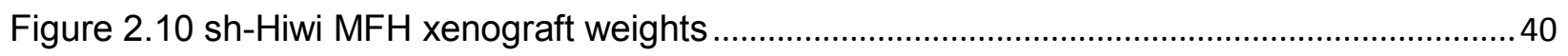

Figure 3.1 Murine Piwi orthologs are not expressed in mesenchymal stem cells....................... 47

Figure 3.2 piRNA expression patterns do not shift in Hiwi-expressing cells ................................50

Figure 3.3 Only two identified piRNA reads correspond to genes ..............................................5 51

Figure 3.42 or $10 \mathrm{~kb}$ upstream analysis reveals 5 piRNAs that lie upstream of genes................53

Figure 4.1 IAP and Line1 transposon expression decreases in Hiwi expressing MSCs .............60

Figure 4.2 Global DNA methylation is increased in Hiwi-MSCs ............................................... 61

Figure 4.3 DNA methyltransferase proteins are elevated in Hiwi-MSCs ....................................62 
Figure 4.4 5-azacytidine treatment restores transposon expression in Hiwi-MSCs in a dosedependent manner.....

Figure 4.5 5-azacytidine treatment reduces colony forming capacity of sarcoma cells

Figure 4.6 Similar gene expression profiles for 5-azacytidine treatment and Hiwi knock down ..69

Figure 4.7 Promoter methylation is not globally shifted during Hiwi knock down 71

Figure 4.8 CpG methylated genes do not correlate to genes expression changes in sh-Hiwi

MFH cells and Hiwi-MSCs 72

Figure 4.9 P-values of differentially expressed genes identified in CpG promoter methylation array

Figure 4.10 Assessment of Hiwi target genes. 75

Figure 4.11 Methylation of Rb1 and Line1 promoters do not change as Hiwi levels change ...... 76

Figure 4.12 Line1 and IAP transposon methylation is unchanged in Hiwi-MSCs .77

Figure 4.13 CDKIs are decreased in Hiwi-MSCs..... 78

Figure 4.14 CDKIs have an inverse relationship with Hiwi in primary human sarcomas..... 79

Figure 4.15 Epigenetic histone marks are unchanged in doxycycline-induced sh-Hiwi-MFH cells

Figure 5.1 A potential model for Piwi and piRNA function in normal development and in cancer

Figure 5.2 Yeast 2-hybrid results indicate H3.3 is a Hiwi binding partner 105

Figure 5.3 A working model for Hiwi-mediated tumorigenesis 109 


\section{Acknowledgements}

This dissertation and the work contained herein would not have been possible without the dedication and support of my thesis mentor, Dr. Igor Matushansky. Igor has not only provided me with any laboratory resource I may have dreamed of, but more importantly, at every step of the thesis process, he provided the generosity of his time. Igor's availability (despite his own very full schedule) to talk with me through the project and its various twists made the development of this project not only smooth, but also a lot of fun. I have greatly enjoyed my time in the lab, not in small part due to the support of such an involved and proactive mentor. Because of Igor's clinical and basic science duties, the lab never strays too far from the overall goal of improving cancer treatments for patients. Working in lgor's lab reminds me every day of the reason that I started a PhD: to contribute to improving human health. For that, I am especially grateful.

The members of my thesis committee have also shown great generosity with their time for this project. Drs. Michael Shen, Alla Grishok, Carlos Cordon-Cardo and Ron Liem have given me valuable input over the years, regarding the development, publication and future of this project. Their availability for both scientific and personal discussions is greatly appreciated.

Much of this work would not have been possible without the daily (and sometimes nightly!) support of my labmates, both in the Cordon-Cardo Lab and in the Matushansky Lab. Many thanks to Dr. Liz Charytonowicz, Todd Hricik, Dr. Mireia Castillo, and Joslyn Mills for lots of technical help (and patience with me!) during the early years of my thesis. The members of the Matushansky Lab: Mel Terry, Drs. Xi Zhang, Dan Han, and Charlotte Alme have been invaluable sounding boards, for both personal and professional topics. A special shout out to Angela Jia and Dr. Fil Dela Cruz, (I'll always remember the Learning Corner with fondness) for being great friends during the roller coaster PhD years-and I hope for many more years to 
come. Lastly, I would be remiss if I did not mention the roommates of apartment 27-D, especially Dr. Geoff Catalano and Sarah Tisdale. I am lucky to have lived with great friends who are as brilliant as they are kind.

Zaia Sivo and Dr. Ron Liem of the Integrated Program have been invaluable advocates for student progress and satisfaction during the PhD training program. I am truly lucky to have been a student in a graduate program that has had my back through every stage of the process. I am particularly grateful to Zaia, for being not only a stellar support in the Integrated Program, but also a caring friend through the years.

I am indebted to my best friend, Heera Basi for the countless hours she has spent on the phone with me during our years of long-distance bi-costal separation. I look forward to being in the same city again soon! Finally, I thank my mom, Farzana, and my brother, Usman, for their unwavering love and support when I told them I'd be moving away for graduate school, and for their continued support during my PhD years. I hope that I've made them proud. 


\section{Dedication}

For my mother, Farzana Siddiqi 


\section{Chapter 1: Introduction}

\section{Sarcomas and mesenchymal stem cells}

Adult tissue stem cells are multipotent stem cells that reside in various tissues of the body throughout life and are able to differentiate into a limited number of specialized tissue types. Included in this group are mesenchymal stem cells (MSCs), which are multipotent cells that can divide to both self-renew and differentiate into various connective tissue lineages such as adipocytic, osteocytic, myocytic and chondrocytic lineages [4]. MSCs can be found in various parts of the body, including bone marrow, muscle and fat [5]. MSCs from bone marrow have been identified as non-hematopoietic stem cells by the lack of hematopoietic lineage markers CD14, CD34 and CD45 [6]. MSCs have been shown to propagate in vitro for several passages without differentiating [7] though they lose their differentiation capacity with prolonged culture in vitro.

A growing body of evidence implicates MSCs as a potential cell of origin during the process of sarcomagenesis. Sarcoma subtype Malignant Fibrous Histiocytoma (MFH; now called High Grade Undifferentiated Sarcoma [HGUS] [8]) can be derived from human MSCs [9] and MFH gene expression profiling parallels that of MSCs. Moreover, inhibition of developmental signaling pathways in MSCs transforms them to form MFH-like tumors in vivo. Conversely, re-establishment of this stem cell developmental signaling in MFH cells allows them to re-establish differentiation capacity down typical mesenchymal lineages. Additionally, specific liposarcoma subtypes have been linked with specific points in adipocytic differentiation of MSCs [10], suggesting a direct relationship between stage of MSC maturation arrest and sarcoma tumor subtype. This allows for the categorization of sarcomas from stem cell to terminal adult tissue based on their differentiation, in a manner similar to categorization of 
hematopoietic stem cell differentiation and lymphomagenesis [11]. Specific points of temporal differentiation along the adipocytic lineage yield gene expression patterns that correspond to poorly differentiated (de-differentiated) and well-differentiated liposarcoma subtypes.

Aberrant MSC differentiation has also been implicated in osteosarcoma formation $[12,13,14]$. Bone formation is dependent on proper coordination between proliferation and differentiation. Jain, et al showed that brief inactivation of the myc oncogene in a conditional transgenic mouse model of osteosarcoma resulted in regression of the tumor and differentiation of the tumor cells into mature osteocytes [15]. Moreover, re-activation of myc induces apoptosis, rather than restoring tumorigenicity. These data suggest that even brief inactivation of an oncogene might alter the genetic and epigenetic context of the cell, rendering terminally differentiated osteocytes less permissive for tumorigenesis than MSCs.

The theory that the inappropriate differentiation of adult tissue stem cells can lead to tumor formation is not a paradigm limited only to mesenchymal stem cells and sarcomas. In fact, a similar concept has been thoroughly examined in hematopoietic stem cells (HSCs). HSCs differentiate into all blood cell types and have been historically well-studied. There is considerable evidence that blood cancers such as leukemia and lymphoma arise from the aberrant differentiation of HSCs [reviewed in 16]. Cell surface antigen profiles of hematological malignancies match those of their progenitor cells at various maturation stages [17]. Additionally, murine transplantation experiments in which leukemias can be initiated from HSC and from more differentiated myeloid progenitors [18] suggests that blood cancer is derived from an adult tissue progenitor population. Similarly, the hypothesis of HSCs as the cell of origin of blood cancers may apply as well to other adult stem cells as the cell of origin for solid tumors such as sarcomas. 
Taken together, these various studies suggest that MSCs may be the cell of origin of some sarcoma tumors, just as HSCs have been implicated in blood cancers [11]. Inappropriate MSC differentiation, as controlled by both genetic assaults and epigenetic modifications, may result in tumor formation. Transformation of a MSC at a specific point in its differentiation down a particular mesenchymal lineage is able to give rise to a corresponding sarcoma subtype (Figure 1.1, adapted from [1]).

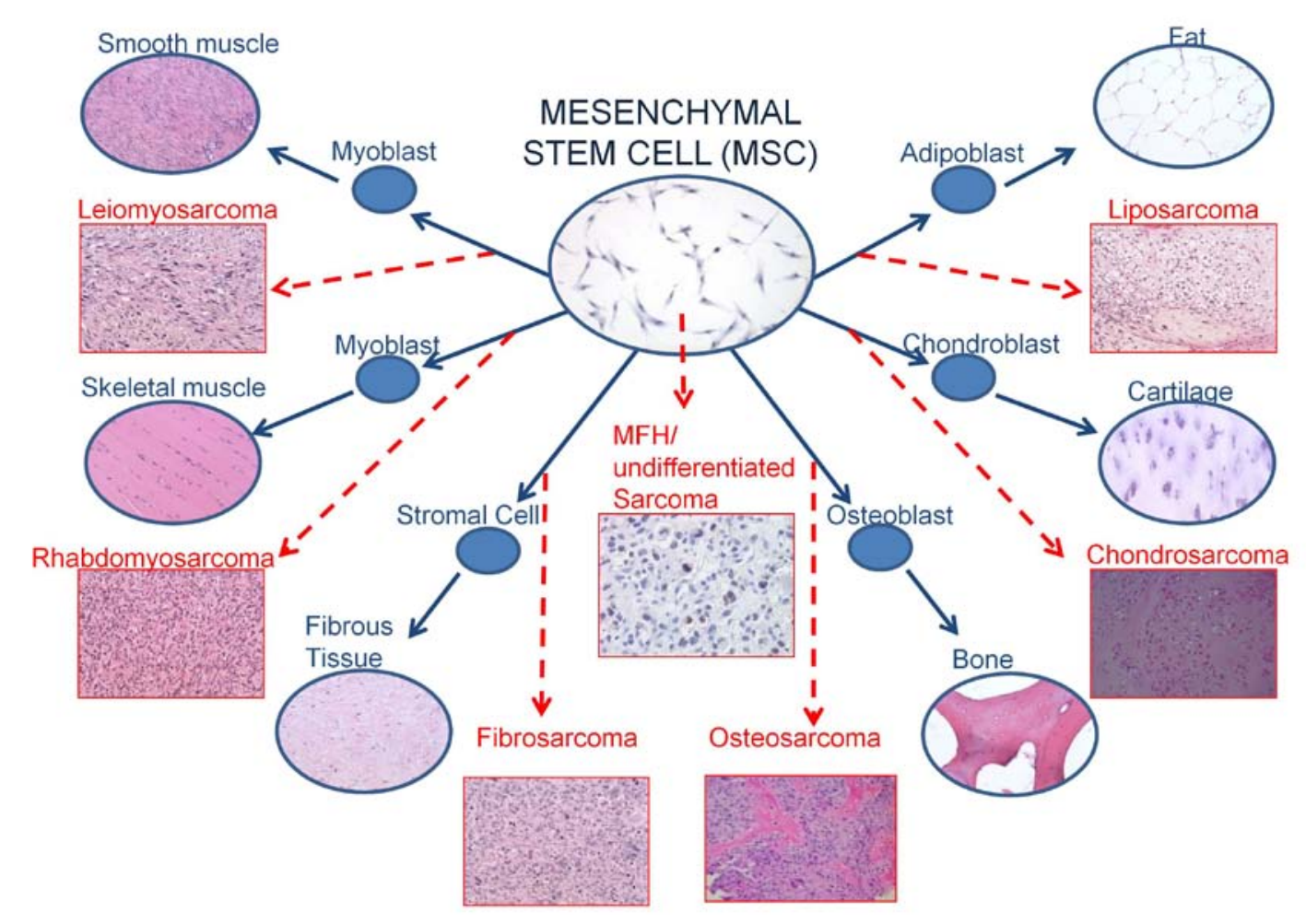

Figure 1.1 Differentiating MSCs transform to develop specific sarcoma subtypes

Transformation of a MSC during its differentiation down a particular mesenchymal lineage can give rise to specific sarcoma subtypes. Image adapted from Charytonowicz and colleagues [1].

The genetic and epigenetic changes that occur during the differentiation of stem cells can be studied using mesenchymal stem cells (MSCs) in vitro. The well-characterized in vitro differentiation of MSCs can be tracked with relative ease in the laboratory. Additionally, their 
corresponding cancer, sarcomas, serve as an excellent solid tumor model, both in vitro and in vivo, for examining the genetic lesions and/or chromatin changes which occur during tumorigenesis.

\section{Stem cell genes in sarcomas}

Global alterations found in many cancers include hypomethylation of DNA [19] and hypoacetylation of histones. In addition to gene-specific hypermethylation of DNA, these alterations lead to oncogene activation (R-ras, cyclin D2) and tumor suppressor silencing (RB1, p16). Cancer has both a genetic and epigenetic basis, where the latter can serve as an alternative to genetic modification. It is possible that in cancer, reversible epigenetic gene repression is replaced by aberrant permanent silencing. Furthermore, if the altered cancer cell is an early progenitor cell, then it is locked in a state of perpetual (and aberrant) self-renewal.

A variety of genetic and epigenetic lesions occur during the transformation of MSCs to become sarcomagenic. Recent report finds that K-Ras expression and p16 disruption in discrete subsets of skeletal muscle progenitors gives rise to distinct subtypes of sarcomas [20]. Moreover, gene expression profiling shows that oncogenes such as Ras are disrupted in both murine and human sarcomas. P53 and Rb1 deficiencies are also commonly found in sarcomas, and MSCs have been confirmed as a preferred target for initiation of sarcomas with these deficiencies, in line with our previous data implicating MSCs as the cell of origin for sarcomas [21]. The Ewings sarcoma tumor subtype is usually associated with a EWS-FLI fusion gene and this fusion is thought to induce expression of embryonic stem cell genes Oct4, Sox2 and Nanog in pediatric MSCs [22]. Taken together, these data implicate a variety of genetic alterations in the initiation and progression of sarcomas, including many stem cellassociated genes. 
Specific epigenetic alterations correlate with sarcoma tumors as well, though more studies are needed to thoroughly understand the role of combinations of chromatin markers on tumor progression. Sarcomas show disruption of wildtype, non-tumor DNA methylation patterns. In vitro work has shown that osteosarcoma cell lines display aberrant DNA hyper- and hypomethylation [23]. We have previously shown that epigenetic changes are important for sarcoma differentiation [9]. Sequential chromatin immunoprecipitation (ChIP) experiments reveal that MSCs differentiated along the osteogenic lineage contain me-H3K4, the classical active chromatin mark, at the promoter regions of both c-myc and cyclin D1 (downstream Wnt/ßcatenin signaling targets) bound to $\beta$-catenin. Wnt/ $\beta$-catenin signaling promotes osteogenic differentiation [24], in agreement with this active me-H3K4 chromatin mark. Additionally, MFH cells with re-established stem cell developmental signaling that are differentiated along the osteogenic lineage reveal the presence of the active chromatin mark. This is similar to MSCs differentiated along the same lineage, suggesting that MFH cells may originate from MSCs and could be re-directed along a terminal cell lineage to regain wildtype chromatin patterns. Conversely, Wnt/ $\beta$-catenin signaling is known to be inhibitory for adipocytic differentiation [25], and in MSCs differentiated along this lineage, the promoter region of cyclin D1 bound to $\beta$ catenin contains the repressive me-H3K27 mark and loses the active me-H3K4 mark. Taken together, these results indicate that sarcomas have distinct epigenetic marks which can be modified not only to differentiate them into mesenchymal lineages but also to recapitulate wildtype chromatin patterns on target genes.

While certain epigenetic chromatin modifications, such as DNA and histone methylation, have been studied in sarcomas, there are still additional chromatin marks that remain unexplored. Importantly, histone acetylation in sarcomas has yet to be thoroughly explored and will certainly yield greater insights into the epigenetic implications of sarcoma development and progression. There are also several genetic alterations found in sarcomas, including the 
expression of stem cell genes. In line with this, the Piwi family of stem cell maintenance genes has recently been found to be highly expressed in soft tissue sarcomas $[26,27]$, perhaps locking a sarcoma progenitor cell in a state of perpetual (and aberrant) stem-like self renewal.

\section{Piwi proteins}

\section{The role of Piwis during development}

Piwi family proteins have been studied in a variety of model organisms, including fly (Piwi), mouse (Miwi), and human (Hiwi) models. Organisms in which Piwi family members have been identified are listed in Figure 1.2, including Piwi homolog names. Across model systems examined, Piwi family member expression is largely limited to the germline (with the exception of one report which finds Hiwi in human hematopoietic progenitors [28]) [2,29,30]. In the germline, Piwis are thought to be essential for stem cell self-renewal. The founding member of the Piwi-subfamily was identified in Drosophila in a transposon-mediated random gene disruption strategy (i.e., single P element enhancer-trap) designed to unmask genes involved in germ stem cell division [31]. A specific group of male Drosophila showed small testes and the gene responsible was termed "P-element induced wimpy" testes - Piwi. On further analysis, both male and female Drosophila sterile mutant gonads with inactive piwi exhibited a failure of germ stem cell division suggesting that piwi function is required to maintain and/or allow for germline stem cell division and differentiation. Conversely, over-expression of Piwi in the soma expands the stem cell domain and increases stem cell numbers [32,33]. Piwi has been detected in ovarian germline cells as well as somatic cells that maintain germ stem cell identity in testes $[32,34,35,36]$. These data indicate that somatic piwi regulates germ stem cell division via an intercellular mechanism and raises the possibility that Piwi may affect even somatic stem cell divisions. 
Down-regulation of Prg-1, the C. elegans homolog of Piwi, results in decreased germline stem cell proliferation and decreased fertility [32,33]. The Zebrafish Piwi homologue Ziwi is detectable in both testes and ovaries and loss of Ziwi results in loss of germ cells [37]. Zili, the other Piwi homolog in Zebrafish, is expressed in primordial germ cells of the developing embryo [38]. The Arabidopsis (plant) Piwi homologue, Zwille, is critical for shoot meristem selfperpetuation $[39,40,41]$. The Planarians (flat worms) have three Piwi homologues-- Smedwi-1, Smedwi-2, and Smedwi-3 --expressed mainly in neoblasts (planarian somatic stem cells). Down-regulation of Smedwi-2 and Smedwi-3 blocks all cellular regeneration, not just that of germ cells. [42,43]. Loss of Mili and Miwi, mouse orthologs of Piwi, in transgenic mouse models results in a complete spermatogenesis block $[44,45]$. Taken together, these data suggest that across model organisms, Piwi proteins are essential for stem cell maintenance (both somatic and germ cell).

\begin{tabular}{|c|c|c|c|c|c|c|c|c|}
\hline Organism & Arabidopsis & Plenarians & Drosophila & Zebrafish & C. Elegans & Mice & Rats & Humans \\
\hline $\begin{array}{c}\text { Homolog } \\
\text { names }\end{array}$ & Zwille & Smedwi-1 & Piwi & Ziwi & Prg-1 & Miwi & Riwi & Hiwi (Piwil1) \\
\cline { 2 - 8 } & Smedwi-2 & Aub & Zili & Prg-2 & Miwi2 & Hiwi2 (Piwil4) \\
\cline { 2 - 9 } & $\begin{array}{c}\text { Smedwi-3 } \\
\text { Loss of } \\
\text { meristem } \\
\text { stem cells }\end{array}$ & $\begin{array}{c}\text { Legeneration of } \\
\text { tissues }\end{array}$ & $\begin{array}{c}\text { Loss of germ cells } \\
\text { (in ovary and } \\
\text { testis) }\end{array}$ & $\begin{array}{c}\text { Loss of } \\
\text { germ cells }\end{array}$ & $\begin{array}{c}\text { Decreased stem } \\
\text { cell proliferation; } \\
\text { Decreased } \\
\text { fertility }\end{array}$ & $\begin{array}{c}\text { Spermatogenesis } \\
\text { block in Miwi null, } \\
\text { Mili null and Miwi2 } \\
\text { null males; females } \\
\text { normal }\end{array}$ & Hili (Piwil2) & Piwil3 \\
\hline
\end{tabular}

\section{Figure 1.2 Piwi homologs across organisms}

Piwi homologs are present in a variety of model organisms, including plants and primitive flatworms. This non-exhaustive list includes names of known homologs within an organism. In all organisms examined, loss of Piwi homologs results in a loss of stem cells. 


\section{Mechanisms of Piwis}

Structurally, Piwis are members of the Paz-Piwi Domain (PPD) family. PPD family members share a central Paz domain, which is a small RNA binding domain, and a C-terminal Piwi domain, which is catalytic and anchors the 5' end of guide RNA. The PPD family is divided into the Ago and Piwi subfamilies (Figure 1.3, adapted from [2]). PPD family members are part of the small RNA-mediated gene silencing machinery. In small RNA-mediated gene silencing, small non-coding RNAs act as sequence specific silencers of target genes. Short-interferring RNAs (siRNA) and microRNAs (miRNA) are processed from long primary transcripts via Dicer and then exert their gene silencing effects by forming a complex with a multi-protein (including Dicer) RNA-induced silencing complex (RISC). The RISC always contains a member of the PPD family, usually of the Ago subfamily. This complex interacts with specific mRNA targets to target their degradation, thus inducing post transcriptional gene silencing (PTGS). 


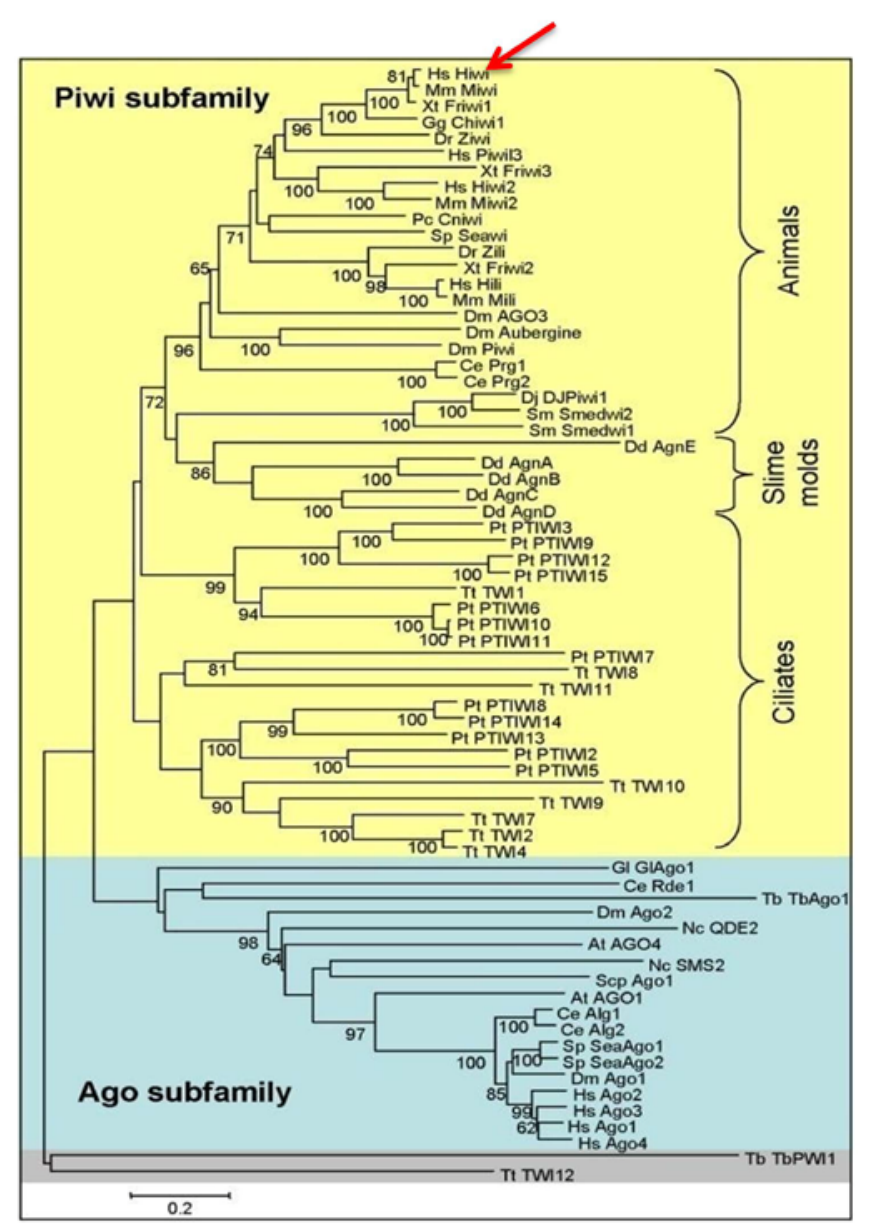

Figure 1.3 The PPD family is divided into the Ago and Piwi subfamilies

The PPD family is divided into the Ago and Piwi subfamilies. Hiwi is the human homolog of the Piwi subfamily (red arrow). Image adapted from Seto and colleagues [2].

Interestingly neither Piwi, nor its two Drosophila homologues - Aubergine and Ago3 (the latter despite the nomenclature belonging to the Piwi subfamily) had been found to interact directly with either miRNAs or siRNAs despite the presence of a conserved Piwi domain common to both subfamilies, suggesting the presence of a third class of small RNAs. In fact a detailed analysis of small RNAs in Drosophila during development did uncover a third group of small RNAs (24-29nt) [46] which are longer than either miRNA or siRNA., have a bias for a 
uridine at their 5' end and are 2'-O-methylated at their 3'end [47,48,49,50,51]. This novel class of small RNAs has been shown to be Dicer1 and Dicer 2 independent [52]. Since these small RNAs corresponded to genomic areas containing highly repetitive and transposon sequences [46], they were termed repeat associated small interfering RNAs - rasiRNAs. Subsequently, rasiRNAs in Drosophila germline cells were shown to associate specifically with Piwi and Aubergine and to be critical for silencing transposon elements [52]. Furthermore, endogenous Piwi immunopurified from Drosophila ovary was shown to exclusively associate with rasiRNAs [35]. rasiRNAs associating with Aubergine or Piwi corresponded to the antisense strand of retrotransposons, while Ago3-associated rasiRNAs were derived mainly from the sense strand. Follow up studies have recently identified rasiRNAs in Drosophila somatic cells as well [53]. Due to their specific association with Piwis, rasiRNAs have been renamed as piwi-interacting, or piRNAs. piRNAs have been identified in the germline of a variety of model organisms, including planarians[42,43], Drosophila [46], zebrafish [37], mice [54] and humans [54].

Although siRNA and miRNA are thought to exert their gene silencing effects posttranscriptionally, there is evidence that piRNAs/Piwis can exert their transposon silencing effects through either PTGS or through epigenetic/transcriptional mechanisms.

\section{Post-transcriptional transposon silencing via piRNAs and biogenesis of piRNAs}

A proposed post-transcriptional transposon silencing mechanism of piRNAs has been proposed in which long primary piRNA transcripts (derived from transposon regions) are alternately cleaved by Piwi-piRNA complexes to generate piRNAs and silence transposons. This is termed the ping-pong cycle (Figure 1.4, adapted from [3]).

Since all three Drosophila Piwi homologues were shown to have endonuclease activity, a model was proposed in which the $5^{\prime}$ end of piRNAs is determined and cleaved by AGO3-piRNA complexes, and the $5^{\prime}$ end of AGO3-associated piRNA is determined by Aub- and Piwi-piRNA 
complexes through a similar piRNA-guided cleavage event [34]. In essence, in this ping-pong cycle of cleavage and alternate recognition of sense and antisense, primary piRNAs (believed to originate from long single-stranded precursors that correlate to transposon repeats) can be amplified if complementary transcripts are available. Amplification initiates when the endonucleolytic activity of Piwi proteins is used to cleave complementary transcripts which induces the formation of a new, secondary piRNA with its $5^{\prime}$ end precisely at the cleavage site. The secondary piRNA can in turn regenerate the initial piRNA by cleavage of its complementary target [36]. This "post-transcriptional gene silencing" mechanism of action suggests that transposon transcripts are cleaved to induce their silencing and generate piRNAs.

The ping-pong cycle is thought to exist in mice as well. Association of Piwi mouse homologues Miwi and Miwi2, was shown to be necessary for their cytoplasmic localization and participation in the ping-pong mechanism of piRNA biogenesis. Furthermore, Miwi2 was shown to assume the functional role of Piwi in the "ping-pong" cycle of piRNA biogenesis $[3,55]$. Finally all three Piwi homologue knockout mice exhibit defects in meiosis which may or may not be related to translational repression $[54,55,56,57]$.

Taken together, these data suggest that an alternate cleavage mechanism for piRNA biogenesis might also serve in some model organisms to silence transposons posttranscriptionally. 


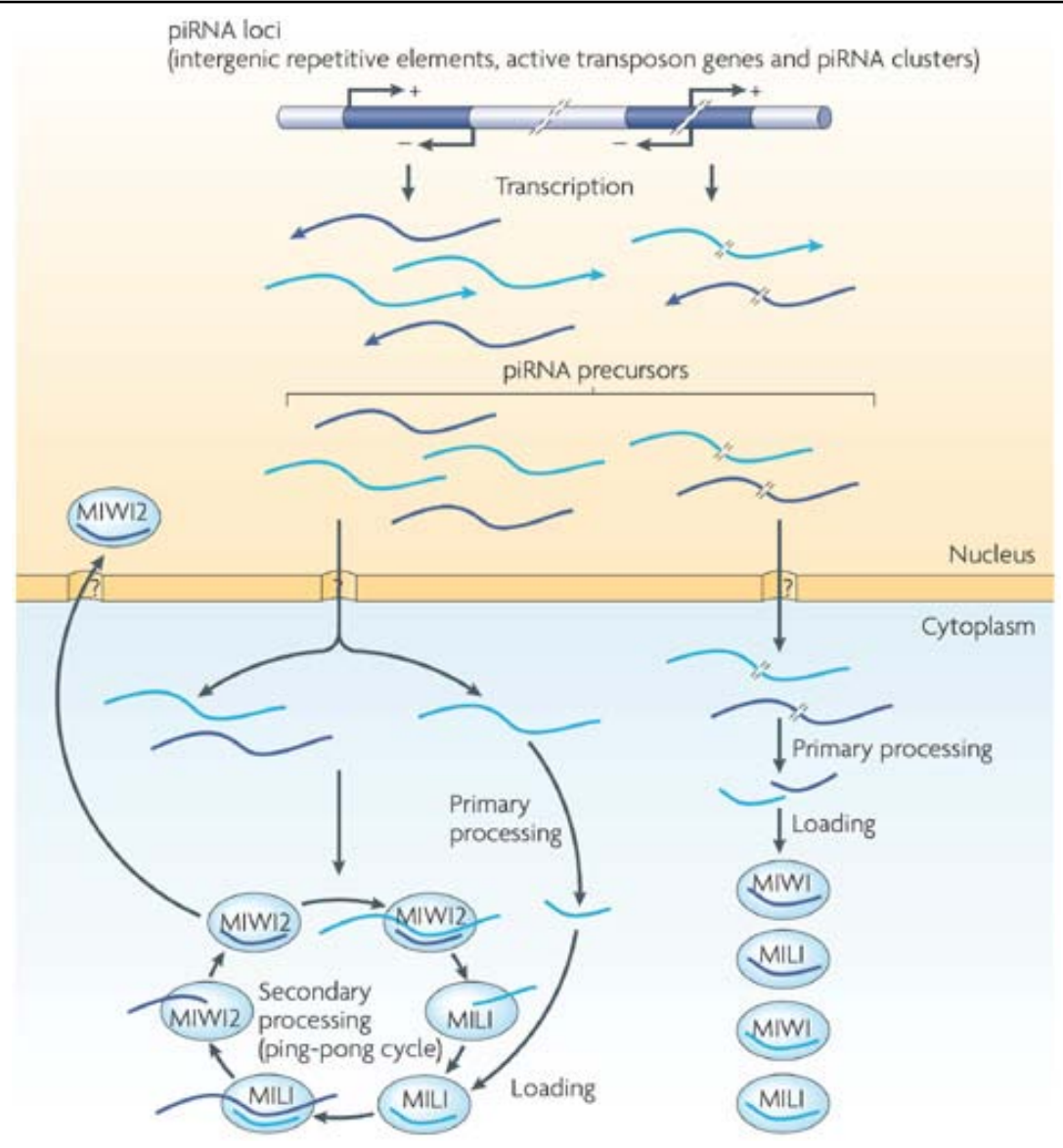

Figure 1.4 Ping-pong cycle of piRNA biogenesis

Piwi homologs bind and catalyze cleavage of a long piRNA precursor and target transcript (referred to as a ping pong cycle) and induce target transcript cleavage in a post-transcriptional silencing mechanism. Piwis/piRNAs are thought to be shuttled in and out of the nucleus. Figure adapted from Kim and colleagues [3]

\section{Epigenetic/transcriptional transposon silencing via piRNAs}

There is a growing body of evidence from a variety of model systems that suggests piRNA-

Piwi pathway components silence transposons in a chromatin-associated mechanism

(epigenetic/transcriptional mechanism) in order to maintain genomic integrity. piRNA-Piwi

complexes are thought to ensure stem cell maintenance by inhibiting transposon migration

$[36,58]$ during early development via an indirect (since Hiwi has no known direct chromatin 
modifiying function) up-regulation of epigenetically based silencing machinery (i.e., DNA methylation) $[55,59,60]$. Specifically, previous studies have shown that transposon-specific DNA-methylation was reduced and transposon activity was elevated following silencing of Hiwi (or its orthologs). Although transposons promote evolutionary diversity in lower organisms, their unchecked migration in higher organisms can result in disruption of genomic integrity [61] and thus Piwi proteins may have developed as an evolutionary defense system for multi-cellular species.

Piwi-associated piRNAs correspond to all types of genomic sequences [62]. One third of them correspond to unique sequences in the genome, including gene-coding sequences and intergenic sequences. The remaining two thirds are transcribed from repetitive sequences. In fact, most of the repetitive piRNAs are derived from transposon sequences that account for only about $10 \%$ of the fully assembled euchromatic genome. These piRNAs are especially overrepresented in retrotransposon encoding regions. Large numbers of Piwi-associated piRNAs are mapped into pericentromeric regions, subtelomeric regions, and telomeres. This broad distribution of piRNAs in nongene coding regions implicates their potential role in epigenetic regulation $[62,63]$.

Even in mice where Piwi orthologs have been more extensively studied in terms of DNA methylation $[55,59]$ the exact mechanism by which Piwi family members mediate DNA methylation during normal embryological development is still unclear.

\section{The role of Piwi in human cancers}

Given the importance of Piwis in silencing transposon migration in order to maintain genome integrity, and given their exclusive stem cell expression, it is surprising that a number of reports find that Hiwi is aberrantly expressed in all cancers examined thus far. Hiwi is highly expressed in diverse cancers, including seminomas [64], gliomas [65], squamous cell carcinomas [66], pancreatic [67], liver [68], and gastric [69] cancers. Work from Taubert and 
colleagues $[26,27]$ finds that Hiwi is highly expressed primary soft tissue sarcomas, where higher Hiwi mRNA levels were predictive of worse clinical outcomes. These data lead to an obvious conundrum: why would a gene that is critical for maintaining genome integrity during development be highly expressed in cancer? Since the above studies focused exclusively on Hiwi expression levels in cancer cells, mechanistic insight into Hiwi's role in tumorigenesis remains completely unexamined.

\section{Piwi-interacting RNAs in non-human models}

A large focus of piRNA research has been dedicated to elucidating their role in germline stem cells. piRNAs were isolated from rodent testes by 4 different groups simultaneously $[54,56,57,70]$, suggesting that this novel class of small RNAs were specific to male germline stem cells. Additionally, the zebrafish Piwi ortholog, Ziwi, is expressed exclusively in the gonad [71], further suggesting that the piRNA-Piwi pathway is specific to the germ-line. Analysis of mouse oocytes revealed no small RNAs that correspond to piRNAs, further strengthening the assertion that piRNAs played a specific role in the male germline.

The questions of piRNA function in all tissues in which they have been identified remain open, although work from germline stem cells in both Drosophila and mouse models partially answer these questions. Most piRNAs are not complementary to the mRNA of potential target genes (unlike miRNAs), further supporting the hypothesis that piRNAs are more likely to be involved in chromatin-associated regulation rather than post-transcriptional regulation [72]. While many mechanistic details remain unclear, there is mounting evidence that piRNAs (and Piwi family proteins) mediate transposon silencing via chromatin-assocaited (epigenetic), rather than post-transcriptional mechanisms.

Work in Drosophila implicates piRNAs in using epigenetic mechanisms to exert their gene expression effects. The piRNA-Piwi pathway is epigenetically regulated in Drosophila 
[62]: piRNAs have been identified at heterochromatic regions and heterochromatin protein 1a (HP1a) was found to bind to piRNA and Piwi. This work implies that piRNA-Piwi pathway components may form a piRNA complex on chromatin that includes other chromatin modifying agents such as HP1a.

Though mechanistic insights into the function of piRNAs in the germline are limited, work from mouse models of Miwi and Mili (mouse Piwi orthologs) deficiency find that expression of these Piwi orthologs is necessary for spermatogenesis [44,73]. Upon knock out of Mili or Miwi, transposon expression is activated [45]. Moreover, de novo methylation of IAP and Line1 transposon region is reduced. Additionally, Mili and Miwi null testes were sequenced to examine changes in piRNA populations. Indeed, piRNAs were dramatically reduced upon Mili knock out, while the miRNA population remained unchanged. These data implicate de novo methylation as an important chromatin-associated (epigenetic) mechanism of the piRNA-Piwi pathway to maintain transposon silencing.

In a complementary study, loss of murine Piwi ortholog Miwi2 in germ stem cells results in increased transposon expression due to a loss of DNA methylation at transposon sites [59]. This epigenetic methylation mark has been further implicated as a mechanism for piRNAmediated transposon silencing in order to maintain genome integrity: Mili and Miwi2 are required for de novo methylation of transposons in the murine testis [45,55], thus showing their importance in establishing rather than maintaining DNA methylation patterns. It is possible that piRNAs might play a role as guide for DNA methylation machinery to transposons. Testes from mice mutant for DNMT3L, a DNA methyltransferase enzyme which regulates de novo methylation, still have a piRNA population. This suggests that not only are Mili and Miwi2 required for de novo methylation of transposon sites, but they also act upstream of DNMT3L [55]. These data are highly suggestive of the importance of epigenetic regulation-rather than 
post-transcriptional regulation—of transposon silencing in order to maintain genomic integrity of the germline.

Interestingly, piRNAs identified in the male germline seem to fall into 2 categories: those that associate with Mili during early spermatogenesis, and those that associate with Miwi during late spermatogenesis [74]. Mili-associated piRNAs are typically 3-6 nucleotides shorter than those that associate with Miwi. These data suggest that these 2 groups of piRNAs might play distinct roles in the germline, although more detailed aspects of these roles are still being investigated.

Given the evidence that Piwis mediate transposon silencing via DNA methylation, it is reasonable to expect Piwi localization to the nucleus. However, many groups have identified cytoplasmic expression of the human Piwi homolog, Hiwi $[69,75,76]$. This incongruity suggests that Piwis (and their corresponding piRNAs) might be shuttled into the nucleus in a carefully regulated manner. A recent review by Siomi and colleagues [77] suggest a model for Drosophila in which mature piRNAs complex with Piwis in the cytoplasm and this Piwi-piRNA complex is transported into the nucleus to carry out its epigenetic silencing functions. Because there is a high degree of homology of Piwis from different organisms, it's likely that this model might be more widely applicable. Alternatively, cytoplasmic Piwis might act through intermediary proteins, such as DNA methytransferases to exert their epigenetic silencing effects.

The germline restriction of piRNAs has recently been called into question by a growing body of evidence examining piRNAs in various cell types. piRNAs have been identified outside germ stem cells, for example, in somatic cells of the Drosophila ovary [78]. A recent study also found piRNAs in neurons of the mouse central nervous system [79]. The authors found that piRNA inhibition results in a decrease in dendritic spine formation, a key measure of synaptic function. A wide-ranging study by Yan and colleagues identified piRNA-like molecules in 
various fly, mouse and macaque somatic tissues, including pancreas and cortex [80]. While these piRNA-like molecules were comparable to piRNAs in most criteria (length, distribution) and Piwi family proteins were identified in the examined tissues, a direct association of these small RNAs with Piwi family proteins was not shown. Taken together, these data suggest that piRNA populations may exist in a variety of somatic tissues. A piRNA population has also been identified in HeLa cells [81], an cervical cancer-derived cell line. Although the piRNA population in HeLa cell is much smaller compared to that found in murine germline stem cells, it is still localized to the periphery of the nucleus, consistent with previous studies [82]. Moreover, Wu and colleagues recently identified a piRNA population in murine mesenchymal stem cells (MSCs) [83]. However, our studies (discussed in Chapters 3 and 4) suggest that piRNAs exist in our murine MSCs without any detectable Piwi homolog expression. Together, these data suggest that, while piRNAs may be present in a variety of stem cell and somatic cell types, Piwi protein expression is much more limited, as it is found only in the germline during normal development.

\section{Piwi interacting RNAs in human cancer}

Since piRNA populations have been identified in a variety of germline and somatic tissues, much work has been undertaken to elucidate the role of small RNAs in cancer. miRNAs and their gene targets have been characterized in a variety of cancers, including breast [84], prostate [85] and liver [86] cancers. However, piRNAs are understudied in cancers and until recently were thought to be entirely absent from cancer cells. Determining the roles of piRNAs in cancers is becoming increasingly important to fully understand the cellular changes that occur during cancer.

While Piwi family members are normally expressed in germline stem cells, their expression is lost during normal development. Thus, all normal adult tissues, including normal 
adult tissue stem cells do not express Hiwi. A growing number studies have found human Piwi homologs Hiwi and Hili to be aberrantly over-expressed in a variety of human cancers $[65,66,67,69,87]$, including cancers originating from the germline (testicular seminomas [64]) and those originating from MSCs (sarcomas [26]). These reports suggest that in a tumorigenic state, a cell's piRNA-Piwi pathway components might be altered.

piRNAs have not been thoroughly studied in cancers, however a few preliminary studies suggest that piRNAs are altered in cancers. A specific piRNA, piRNA 651, has been shown to be aberrantly over-expressed in multiple cancers, including gastric, colon, lung and breast cancer tissues, when compared with normal tissues [88]. Furthermore, inhibition of this piRNA in gastric cancer cell lines (another cancer in which Hiwi is over-expressed [69]) decreased cellular growth. While the growth inhibition effect of the piRNA antagonist was modest, these data are suggestive of an important potential role for aberrant piRNA expression in contributing to over-proliferation in cancer cells.

piRNAs were further investigated in gastric cancer by their detection in peripheral blood from patients [89]. Two specific piRNAs, one of which was piRNA 651, were chosen for evaluation and both were significantly lower in peripheral blood from gastric cancer patients, compared to healthy controls. This report suggests that piRNA detection might be a valid biomarker for identifying circulating gastric cancer cells in the blood. While these data may seem contradictory to the Liu, et al, report which finds high piRNA 651 levels in cancer tissues [69], it is important to note that these are the first studies to examine piRNA expression levels in cancer and much more investigation is necessary, both to validate these studies and to identify piRNA expression in additional cancer types. Importantly, these studies did not provide evidence for a general alteration of the piRNA population in cancer versus normal cells; rather they focused on one of 2 specific piRNAs which may or may not be indicative of the entire 
piRNA population. Because no evidence was given for the rationale behind evaluating these 2 specific piRNAs, the potential alterations in all other piRNAs remain unknown. Knowing how those 2 piRNAs were chosen for further analysis would provide valuable information on the piRNA populations in these cancers, but also on the application of this work to other cancers which have high levels of Hiwi. Further, because no mechanistic insight into the role of piRNAs in cancer has been explored, it is not known if piRNAs might be driving tumorigenesis or if their alteration is merely a bystander effect that is part of the cell's stem cell recapitulation process. While Piwi orthologs have been found to be highly expressed in a variety of cancer types, the presence or absence of piRNAs in a variety of cancers has not been fully explored.

On one hand, Hiwi and its orthologs are critical for maintenance of germline stem cells during normal development. On the other hand, Hiwi aberrant expression has been identified in all cancers examined, including in sarcomas. A potential connection between MSCs, sarcomas, and Hiwi remains unexplored and the role of Hiwi in sarcomas is still unclear. How Hiwi may act appropriately to promote genomic integrity during early development (via transposon silencing) and inappropriately in adult tissues with subsequent tumorigenesis has not been investigated. 


\section{Chapter 2: A novel oncogenic role for Hiwi}

\section{Hiwi in Sarcomas}

Recent studies by Taubert and colleagues $[26,27]$ identified Hiwi mRNA levels to be upregulated in primary soft tissue sarcoma samples. These data were intriguing for a variety of reasons. As a stem cell maintenance gene expressed during normal development, Piwis are not expected to be expressed in the adult organism. Moreover, Hiwi was previously identified only in human hematopoietic progenitor cells [28]. Thirdly, this was the first report of Hiwi expression in sarcomas, although work from others had shown that Hiwi is elevated in gastric cancer [69].

\section{Rationale of the Study}

As described earlier, Hiwi mRNA has been detected at very high levels in primary sarcomas, using a quantitative PCR technique [26]. Moreover, high levels of Hiwi mRNA expression were correlated with a poorer clinical outcome for the patient. We wanted to further explore a possible connection between sarcomas and Hiwi, including a functional role for Hiwi in an aberrant cancer state. Before the completion of our studies, the role of Hiwi in cancer generally, and in sarcomas specifically, remained unexplored areas of cancer/stem cell biology.

\section{Experimental Design}

In order to elucidate a functional connection between Hiwi and sarcomas, we first characterized Hiwi expression at the protein level in primary human sarcoma samples. We then generated both Hiwi over-expression and Hiwi knock down models in vitro. Because previous work from our lab implicates mesenchymal stem cells as the likely call of origin for sarcomas $[1,9,10]$, we generated a Hiwi over-expression model in mesenchymal stem cells and evaluated mesenchymal differentiation capacity and tumorigenesis. To perform converse experiments, we 
generated a conditional knock-down model of Hiwi in a sarcoma cell line, MFH, which expresses Hiwi protein at a very high level. Lastly, we generated a mesoderm-specific transgenic mouse model of Hiwi over-expression in order to determine Hiwi's role in sarcomagenesis in vivo.

\section{Results}

\section{Hiwi is highly expressed in human primary undifferentiated sarcomas}

Following previous PCR-based observations that Hiwi is expressed in sarcomas [27] and that its expression correlates with prognosis [26], we examined Hiwi protein levels via immunohistochemistry (IHC) in a large primary human sarcoma tissue microarray (TMA) composed of numerous soft-tissue sarcomas (previously described by us [9]). The sarcoma TMA contains 12 leiyomyosarcoma cases, 10 high grade undifferentiated sarcoma cases, 10 well-differentiated sarcoma cases, 3 pleiomorphic sarcoma cases, 12 dedifferentiated sarcoma cases, 6 low grade myxoid sarcoma cases, and 6 high grade myxoid sarcoma cases. Each case was present in triplicate. The indicated sarcoma subtypes were scored from 0 to 2 blindly by sarcoma pathologists. To examine the relationship between cellular differentiation and tumor grade, we focused on a panel of liposarcomas, since we have previously shown that high grade undifferentiated sarcomas (HGUS), dedifferentiated liposarcomas, pleomorphic liposarcomas, and well-differentiated lipoarcomas correspond to a gradual adipocytic differentiation spectrum [10]). We noted that Hiwi levels correlated directly with grade and indirectly with tumor cellular differentiation. (Figure 2.1A). Overall Hiwi is expressed at significantly higher levels $(p<0.005)$ in undifferentiated sarcoma subtypes compared with more well-differentiated subtypes (Figure

2.1B). Both tumor grade and tumor cellular differentiation have been shown to correlate with clinical prognosis for sarcomas [90]. These data suggest that Hiwi is associated with the 
undifferentiated mesenchymal tumorigenic state and thus by definition designates a poor prognostic outcome [90].

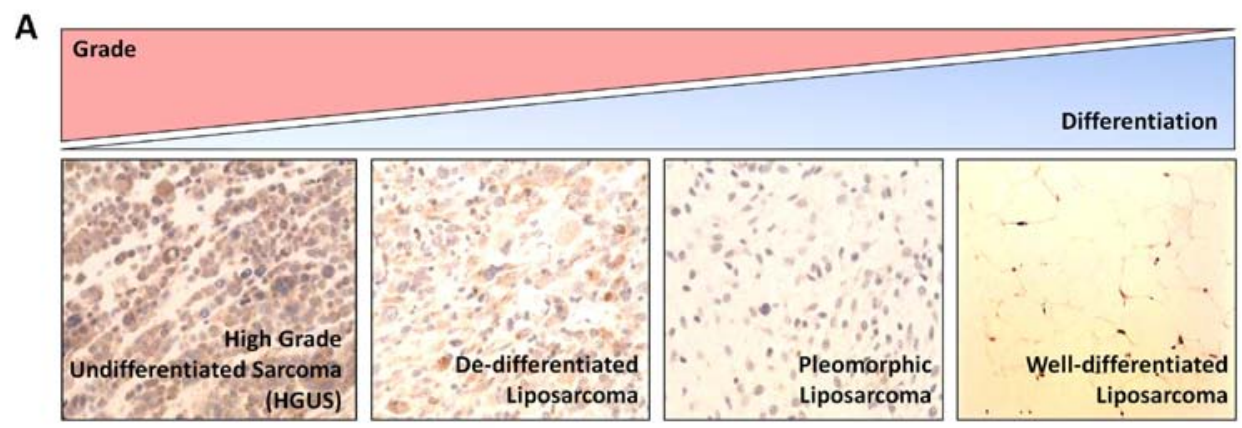

B

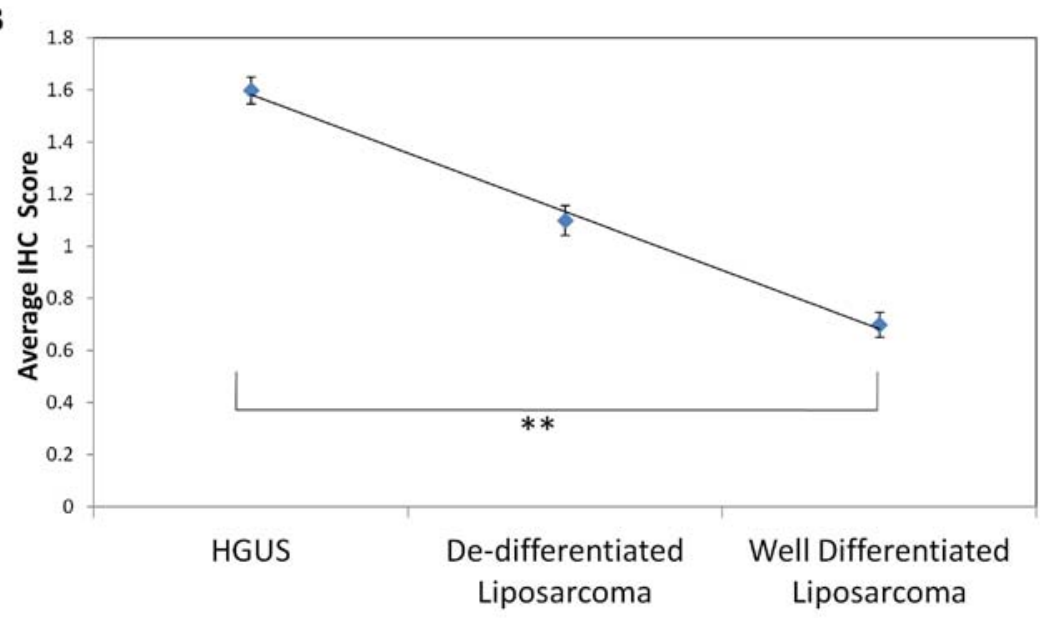

Figure 2.1 Hiwi is highly expressed in human undifferentiated sarcoma samples

(A) Immunohistochemical (IHC) analysis of Hiwi on a human sarcoma tissue microarray (TMA). Ten cases of each subtype (present in triplicate) were scored from 0 to 2 blindly by sarcoma pathologists. Representative pictures are shown. (B) Average scores are plotted here for each subtype. Error bars represent standard error ${ }^{* *}=p<0.005$ by Student's TTest HGUS= high grade undifferentiated sarcoma.

\section{Hiwi is not chromosomally amplified in Hiwi-expressing sarcomas}

We then assayed, via DNA fluorescence in situ hybridization (DNA FISH), for Hiwi chromosomal amplification using the same sarcoma TMA used to validate Hiwi protein levels and correlated Hiwi IHC-based expression to Hiwi chromosomal amplification. Only one case 
out of 45 examined carried a true chromosomal amplification of the Hiwi locus on chromosomal 12 (Figure 2.2). We have assayed for Hiwi amplification using the tissue microarray (Figure 2.1A) and attempted to correlate IHC-based expression to Hiwi amplification. However only one case out of 45 examined carried a true chromosomal amplification of the Hiwi locus on chromosome 12 (Figure 2.2A), a few cases (5 out of 45) had a copy number increase of chromosome 12 (Figure 2.2B) while the majority of cases (39 out of 45), including all HGUS cases, did not have any amplification of chromosome 12 (Figure 2.2C). These data suggest that although Hiwi is expressed at high levels in the most undifferentiated sarcoma subtypes, this is not due to a characteristic amplification of the Hiwi locus on chromosome 12. 
A

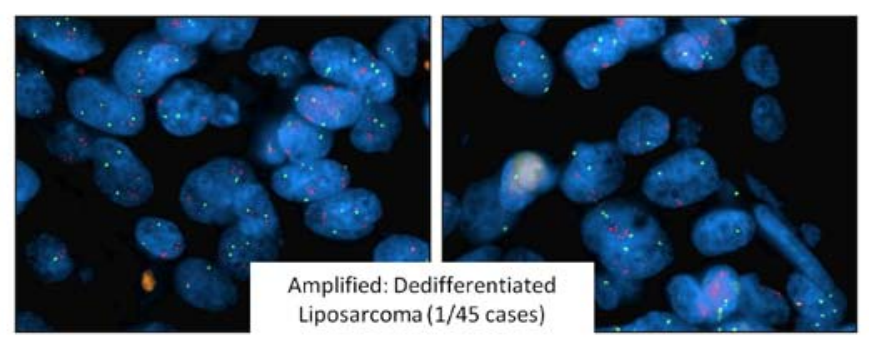

B

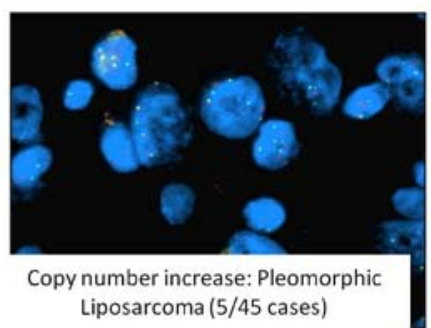

C

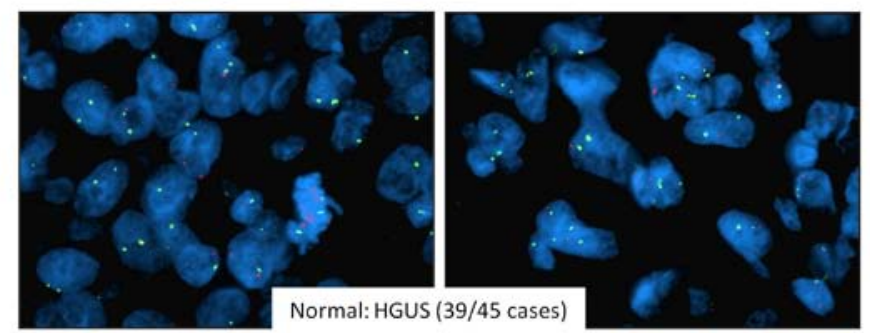

Figure 2.2 Hiwi is not chromosomally amplified in Hiwi-expressing sarcomas

DNA FISH was performed on the human sarcoma TMA, using a probe against the Hiwi locus on chromosome 12. (A) Analysis of the sarcomas reveals only 1 out of 45 cases that has a true amplification of Hiwi, a dedifferentiated liposarcoma. (B) A few cases (5 out of 45) have a copy number increase of Hiwi. (C) However, the majority of cases (39 out of 45), including all HGUS Hiwi-expressing cases, have no chromosomal amplification of Hiwi. Green= centromere 12; Red=Hiwi probe

\section{Generation of Hiwi over-expressing mesenchymal stem cells}

To assess whether Hiwi merely associates with inhibition of differentiation and tumorigenesis, or whether Hiwi may directly inhibit differentiation and promote sarcomagenesis, we expressed Hiwi in sarcoma precursors (i.e., mesenchymal stem cells) using a lentiviral construct. In order to validate our Hiwi-MSCs beyond IHC staining, we performed a quantitative RT-PCR (Figure 2.3A) on parental MSCs, MSC5 (a non Hiwi-expressing clone), and Hiwi- 
MSCs 3 and 7 (Hiwi-expressing clones), along with their corresponding no reverse transcriptase controls. Hiwi-MSC 3 and 7 express high levels of Hiwi, as compared to parental MSCs and MSC5. Additionally, we performed western blotting analysis of various clones (Figure 2.3B) obtained during the transfection/infection procedure. MSC2, 5 and 6, along with parental MSCs, showed no Hiwi expression. Hiwi-MSC clones 3 and 7 express Hiwi (middle band; 100kDa). AGS and N87 gastric cancer cell lines have been previously reported [69] to express Hiwi and were used as positive controls. However, Hiwi is more readily detected in N87 at this exposure, and both are expressed at much lower levels compared to either of our Hiwi-expressing clones (Hiwi-MSC 3 and 7). The Hiwi band for N87 control cells may be slightly higher than the MSCHiwi 7 Hiwi band, likely due to technical difficulties with this antibody. This is consistently seen in our western blots using this antibody to detect N87 and clonal Hiwi expression. Because commercially available antibodies for Hiwi are more suited for IHC rather than western blotting, the western blot shown in Figure 2.3B shows non-specific banding and $\mathrm{IHC}$ is preferentially used in the following studies. A high non-specific band at about $115 \mathrm{kDa}$ is consistently seen in our Western blots with this antibody. Additionally, a lower band around $82 \mathrm{kDa}$ is seen in the Hiwi-expressing clones (Hiwi-MSC 3 and 7). This band may represent a degradation product of Hiwi, however its exact identity is unclear. 
A
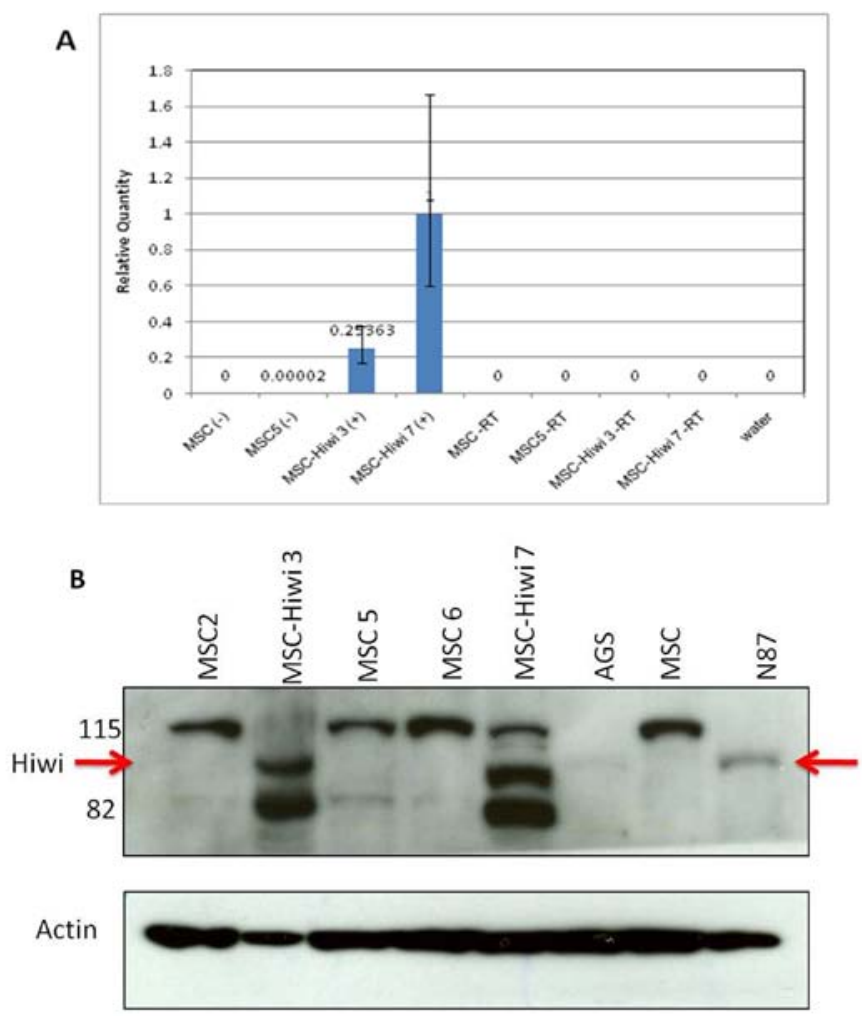

Figure 2.3 Generation of Hiwi-expressing mesenchymal stem cells

(A) Parental MSCs, MSC5 (a clone which is selection-marker resistant but doesn't express Hiwi), and Hiwi-expressing clones 3 and 7 were analyzed by quantitative RTPCR for Hiwi expression. MSC-Hiwi 7 was arbitrarily set at 1. (B) MSC clones were analyzed by Western Blot for Hiwi expression levels. MSC-Hiwi 3 and 7 were positive clones, where as MSC2, 5, and 6 gained selection marker resistance without expressing Hiwi. MSC5 was chosen for further experiments. AGS and N87 gastric cancer cell lines have been previously reported as positive controls for Hiwi expression. A high nonspecific band at about $115 \mathrm{kDa}$ is consistently seen in our Western blots with this antibody. Additionally, a lower band around $82 \mathrm{kDa}$ is seen in the Hiwi-expressing clones (Hiwi-MSC 3 and 7). This band may represent a degradation product of Hiwi, however its exact identity is unclear.

\section{Hiwi over-expression blocks mesenchymal differentiation capacity in vitro}

We first observed that Hiwi-expressing MSCs show a distinct morphology when grown in culture. Compared to parental MSCs which grow in a monolayer, the Hiwi-expressing MSCs grow in a clumped manner where colonies form and cells begin to grow on top of each other 
(top row, Figure 2.4). To assess whether Hiwi merely associates with inhibition of differentiation and tumorigenesis, or whether Hiwi may directly inhibit differentiation, we expressed Hiwi in sarcoma precursors (i.e., mesenchymal stem cells, Figure 2.3). Hiwiexpressing MSCs (Hiwi-MSC 3 and 7) and parental MSCs were cultured in either adipogenic or osteogenic differentiation medium [9] and, as per standards of the field, were assayed for phenotypic maturation with either Alizarin-Red-S for bone/calcium mineralization or with OilRed-O for fat accumulation. Hiwi-expressing MSCs (Hiwi-MSC-3\&7; columns 2 and 3, Figure 2.4) show impaired differentiation into both osteogenic (second row, Figure 2.4) and adipogenic (third row, Figure 2.4) mesenchymal lineages as compared to parental MSCs (pMSC; column 1, Figure 2.4), which readily accumulate mineralized calcium and form adipocytic foci. Calcium matrix formation, as measured by Alizarin-red-S staining, is significantly decreased in HiwiMSCs compared to parental MSCs. Similarly, lipid formation, as measured by Oil-Red-O staining decreased in Hiwi MSCs compared to 25\% parental MSCs. These data suggest that Hiwi expression blocks the capacity of MSCs to differentiate down typical mesenchymal lineages in vitro. 


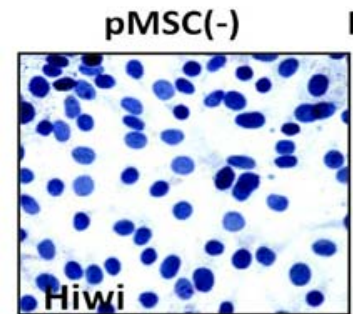

MSC-Hiwi 3(+)
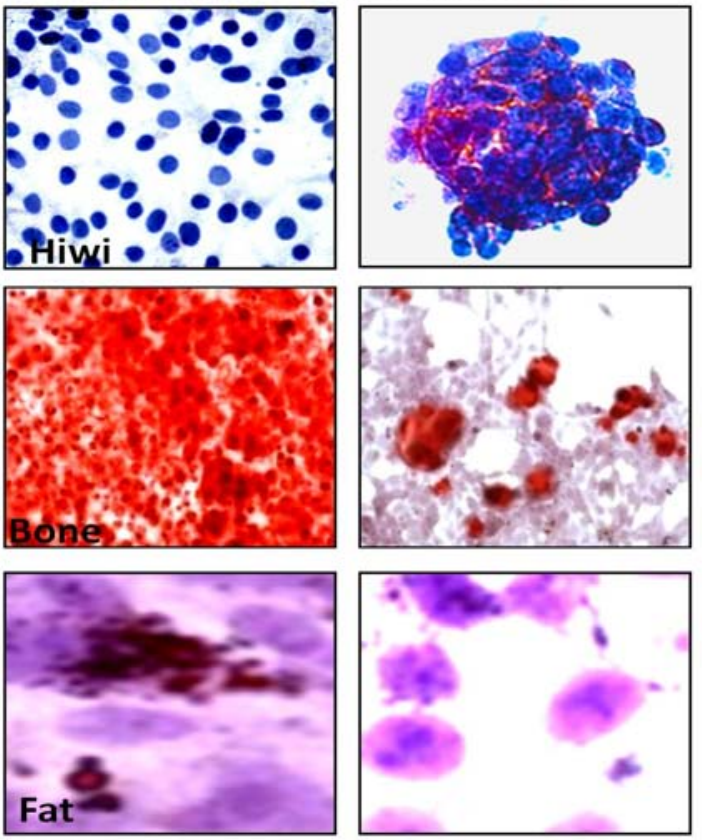

MSC-Hiwi 7(+)
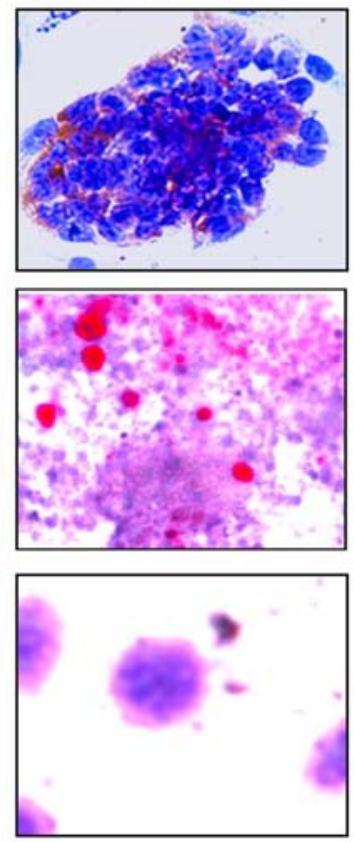

Figure 2.4 Hiwi over-expression blocks mesenchymal differentiation in vitro

Top row: IHC analysis reveals Hiwi-MSC clones (3 and 7) have a distinct clumped morphology and highly express Hiwi. Middle row: Day 21 in osteogenic media. Calcium matrix formation measured by Alizarin Red S stain is decreased in Hiwi-MSCs, compared to pMSC. Bottom row: Day 21 in adipogenic media. Lipid formation measured by Oil Red O stain is decreased in Hiwi-MSCs compared to pMSC. Experiments were performed in triplicate and representative pictures are shown.

\section{Hiwi over-expression transforms mesenchymal stem cells in vivo}

In order to further characterize the effects of Hiwi over-expression in MSCs, we asked whether Hiwi may transform these sarcoma precursor cells to become tumorigenic. To test this hypothesis, we inoculated Hiwi-expressing MSCs (Hiwi-MSC 3 and 7), parental MSCs and a non-expressing, antibiotic resistant MSC clone (MSC5; isolated from our initial transduction) into NOD-SCID-Gamma mice. Tumors formed from MSC-Hiwi 3 and 7 inoculations after five weeks. Morphological analysis (Fabrizio Remotti MD, Department of Pathology, CUMC) showed that 
tumors derived from Hiwi-MSC 3 and 7 inoculations were high grade sarcomas. Further IHC analysis also showed that they expressed Hiwi (Figure 2.5A, bottom row). In contrast to MSCHiwi 3 and 7, parental MSCs and MSC5 formed small fibrous plaques devoid of tumor cells (Figure 2.5A, middle row,). Xenograft weights were quantified (Figure 2.5B) and xenografts derived from Hiwi-MSCs were significantly larger than small calcified nodules found in parental MSCs and in a non-Hiwi expressing clone, MSC5. Taken together, these data suggest that Hiwi is able to transform MSCs to become tumorigenic in vivo. 

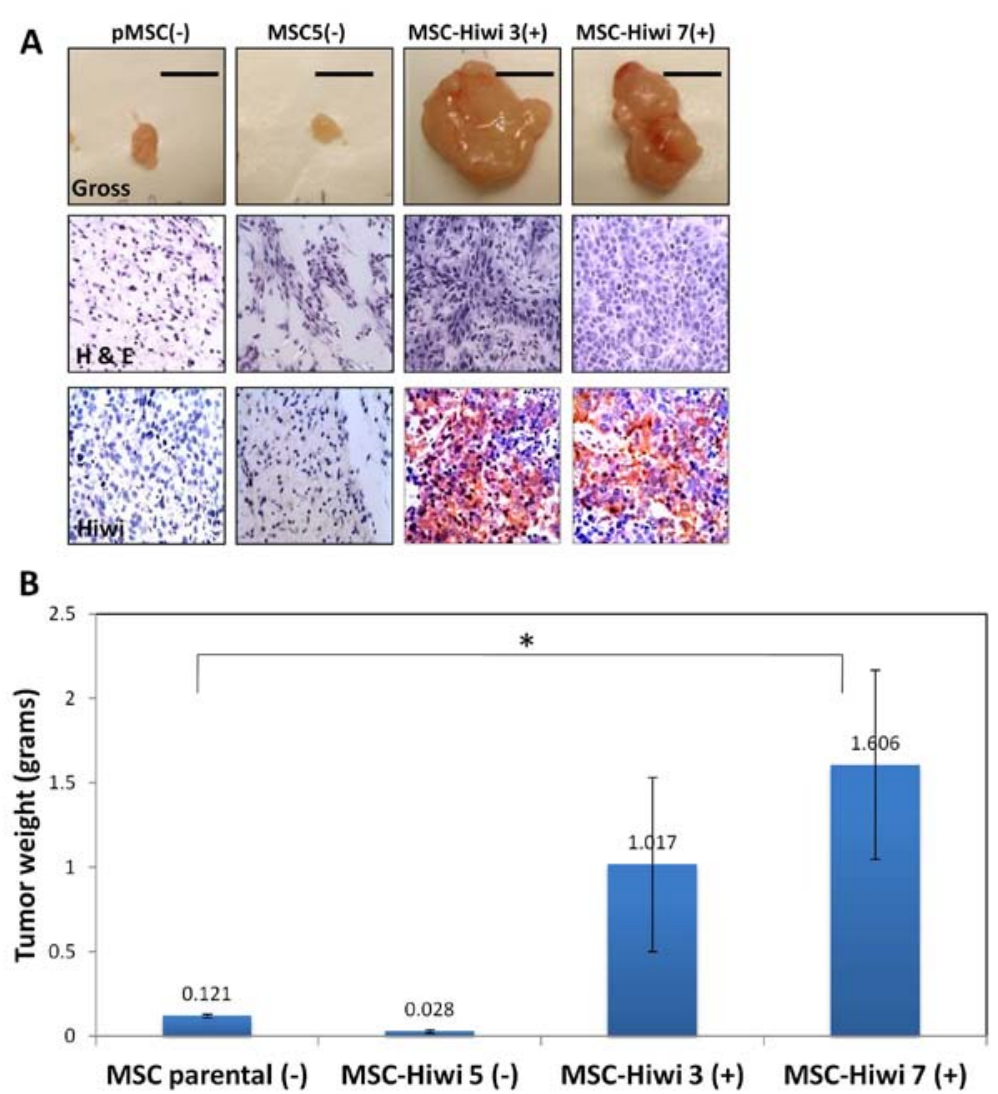

Figure 2.5 Hiwi transforms MSC to become tumorigenic in vivo

(A) Top row: Xenograft tumors derived from Hiwi-MSCs. Middle row: H\&E analysis reveals tumors from Hiwi-MSCs are undifferentiated sarcomas. Bottom row: By IHC analysis, tumors from Hiwi-MSCs continue to express Hiwi. All experiments were performed in 3 mice $(n=3)$. Each mouse was injected with the 2 Hiwi-negative MSCs at the top and bottom of the left flank, and the 2 Hiwi-positive MSCs at the top and bottom of the right flank. (B) Quantification of average xenograft size in grams. Error bars represent standard error and weights are the average of 3 tumors (1 per mouse). * $=p<0.05$ by Student's T-Test

\section{Stem cells are more sensitive to Hiwi-mediated transformation}

To further explore the oncogenic properties of Hiwi we performed classical oncogene cooperation assays in which Hiwi was infected into MSCs and Mouse Embryonic Fibroblasts (MEFs) alone and in conjunction with Ras. Hiwi-transformed MSCs form large colonies $(p<0.05$, compared to untransfected controls), which significantly increase in size and frequency when 
combined with Ras $(p<0.005)$ (Figure 2.6). Interestingly we did not observe a similar transformative effect using MEFs suggesting that Hiwi's oncogenic function may depend on the context of cell type. These results indicate that Hiwi is sufficient to transform progenitor cells into sarcomas. Taken together our data suggest that stem cells may be more sensitive to Hiwimediated transformation, compared to more differentiated cell types. We are aware that certain control experiments must be completed in order to definitively show that progenitor cells are differentially sensitive to transformation via Hiwi, including measuring transfection efficiency and transgene expression levels. However, these are preliminary data which are promising and we are continuing to pursue this line of investigation. Although our data are intriguing, we acknowledge that further studies must be done to establish the sensitivity of stem cell specifically to Hiwi-mediated transformation. This might include similar colony forming assays on additional cell lines (of varying degrees of differentiation) or primary stem cells as well. 


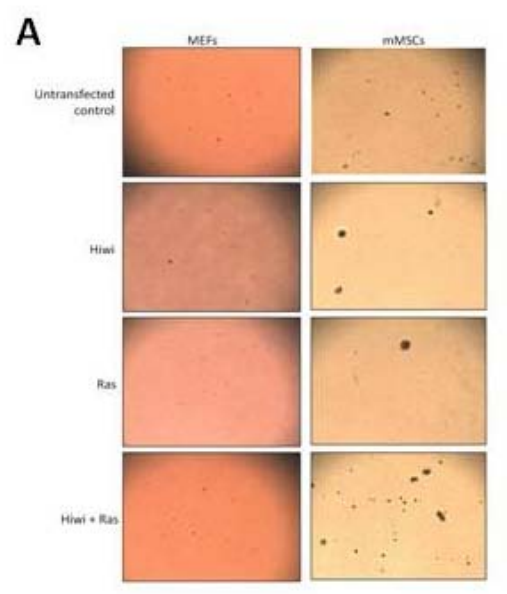

B

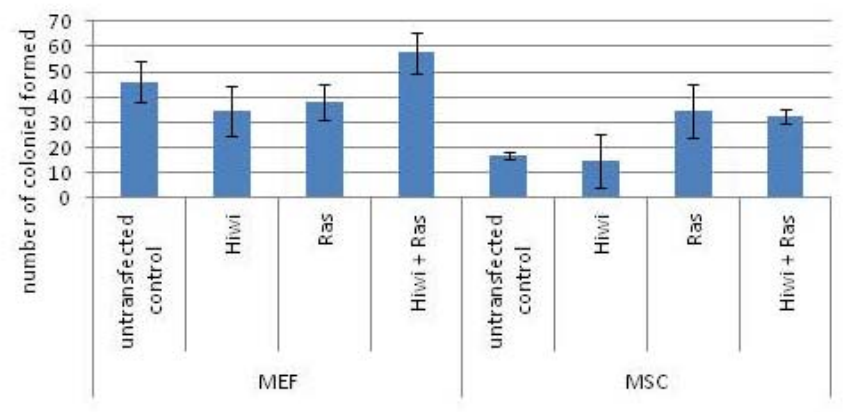

Figure 2.6 Stem cells are more sensitive to Hiwi-mediated transformation

(A) MEFs (left column) or MSCs (right column) were transfected with either Hiwi alone, Ras alone or with both Hiwi and Ras and then put into colony formation assays. At 4 weeks in colony formation assay, both Hiwi alone and Ras alone formed colonies in MSCs $(p<0.05$ compared to untransfected control) and together they formed significantly more colonies $(p<0.005$ compared to untransfected control). No significant changes in colony formation were observed in the transfected MEFs ( $p>0.5$ for all transfections compared to untransfected control). All experiments were performed in triplicate. Representative pictures are shown here all at 20X magnification. (B) Quantification of number of colonies formed during the experiment described in (A). Experiment was repeated in quadruplicate and average number of colonies is presented here. Error bars represent standard error.

\section{A mesoderm-specific transgenic mouse model of Hiwi develops lipomatous lesions}

We further generated transgenic mice expressing Hiwi under the control of the early mesodermally restricted Prx1 enhancer element [91]. The Prx1 enhancer element drives the expression of genes in the developing limb bud mesoderm, but not ectoderm. 51 Prx1-Hiwi expressing progeny have been generated to date. Early sarcoma formation was identified in two (4\%) of 51 Prx1-Hiwi mice at 12 weeks, in a known Prx1 distribution (on the limbs), that contain both well-differentiated and high grade components and express Hiwi (Figure 2.7). No other developmental or pathological abnormalities were observed in these Prx-Hiwi transgenic mice. No sarcoma formation was found in wildtype, Hiwi-non-expressing littermate control mice. 
Although we realize this is a low tumor incidence, it is still higher than the tumor incidence rate found in mice bearing the deletion of the retinoblastoma tumor suppressor under the same promoter (which is no tumor formation at all) [92]; suggesting to us that the low incidence rate may under-represent Hiwi's tumorigenic potential in this model. The organs of Prx-Hiwiexpressing mice, both those that form tumors and those that do not, show no discernible abnormalities by H\&E (reviewed by pathologists Drs. Mireia Castillo-Martin and Fabrizio Remotti). There was no evidence of a pre-neoplastic state in Prx-Hiwi mice which do not form tumors, based on gross dissection and H\&E review. While these mice have not been further characterized, we are working on generating the next iteration of the Hiwi transgenic mouse model, described in detail in chapter 5.
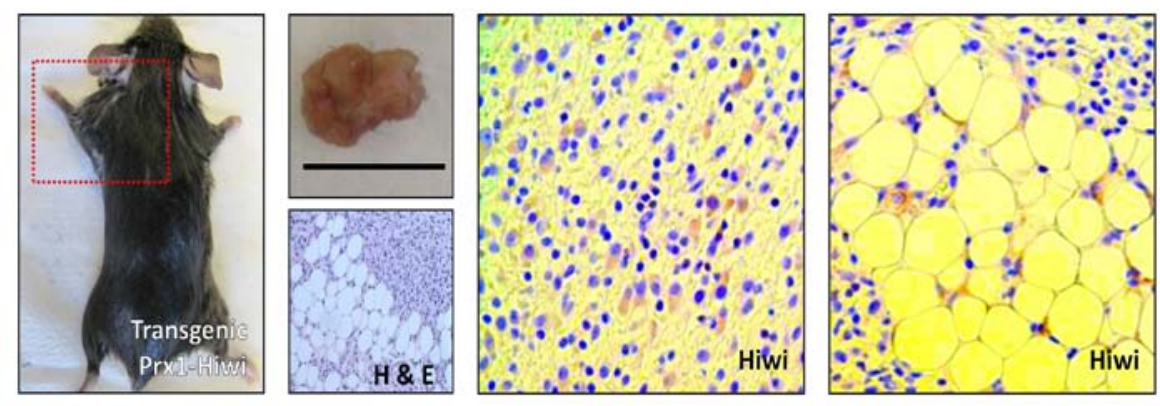

Figure 2.7 Mesoderm-specific transgenic mouse model of Hiwi over-expression

Transgenic mouse model of Hiwi forms sarcomas (left panel) with both well-differentiated and poorly-differentiated sections (H\&E panel) and continues to express Hiwi (right panels). No other abnormalities are found in these mice.

\section{Generation of short-hairpin Hiwi knock down sarcoma cells}

Having shown that Hiwi expression in sarcoma precursors leads to sarcomagenesis, we then asked if Hiwi is necessary for maintenance of the sarcoma phenotype. We have previously 
characterized a panel of human sarcoma cell lines [93]. Previously reported gene expression analysis predicted that the high grade undifferentiated sarcoma cell line, MFH, expressed high levels of Hiwi at the RNA level. We validated the undifferentiated sarcoma cell line, MFH (previously characterized by us [9,93], endogenously expresses Hiwi mRNA at very high levels via quantitative RT-PCR (Figure 2.8A). We then assessed Hiwi protein expression via IHC in MFH cells as compared to other sarcoma cell lines (Figure 2.8B). Both RT-PCR and IHC confirm our initial gene expression analysis and identify MFH as a sarcoma cell line that endogenously expresses Hiwi.

We then engineered MFH cells to express doxycycline-inducible Hiwi-short hairpin; (dox-indsh-Hiwi MFH; Figure 2.8C, top left panel). Both parental MFH cells and sh-scramble MFH negative control cells continue to express high levels of Hiwi. In contrast, doxycycline-treated sh-Hiwi MFH cells have dramatically reduced Hiwi levels (Figure 2.8; Clones C and E were chosen for further analyses). We recognize that knock-down data are often shown by western blotting, and here we use IHC to verify Hiwi knock-down. IHC analysis was chosen over western blotting, since commercial antibodies work more reliably in IHC-based assays. 

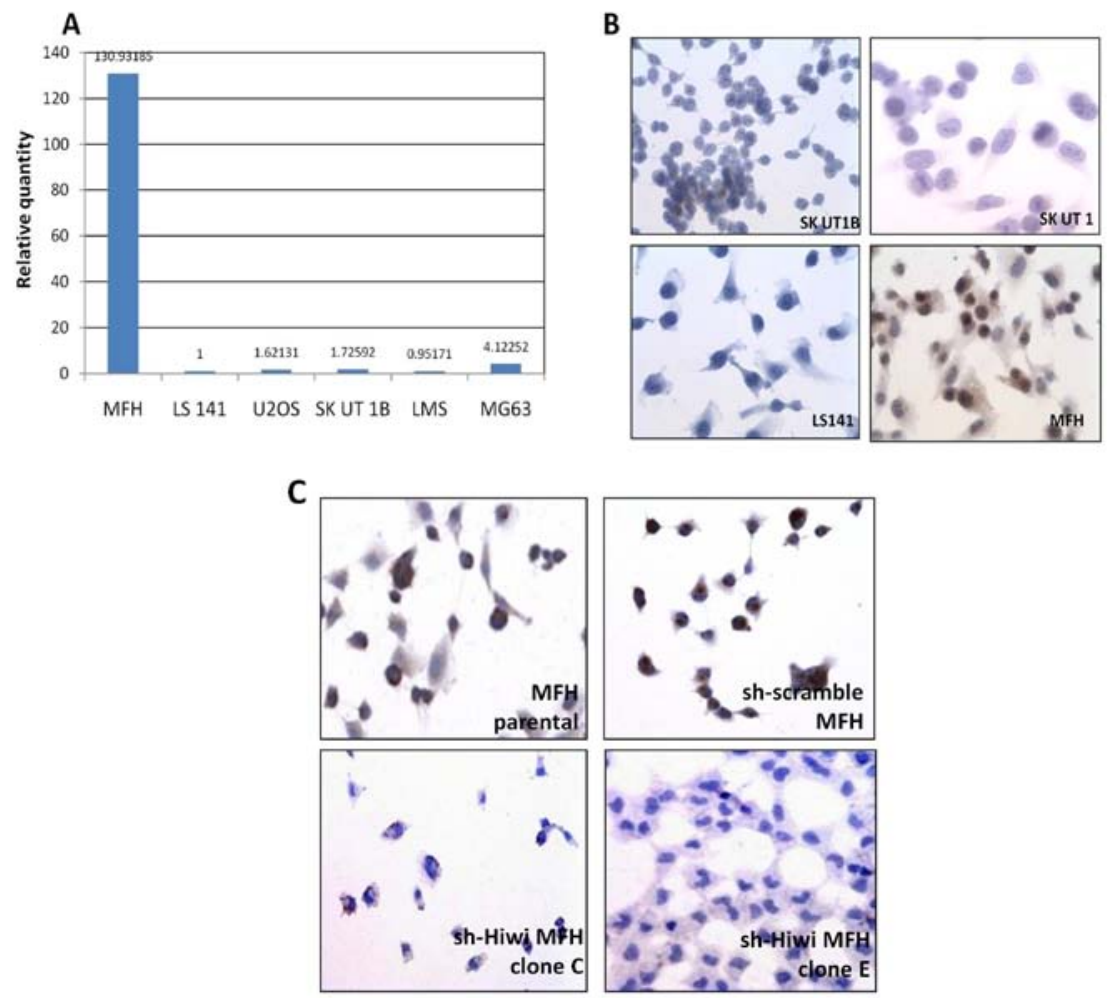

Figure 2.8 Generation of short-hairpin Hiwi knock down sarcoma cells

(A) Quantitative RT-PCR of Hiwi mRNA in a panel of human sarcoma cell lines. (B) Immunohistochemical analysis of Hiwi protein in a panel of human sarcoma cell lines (C) Verification of inducible sh-Hiwi MFH clones by immunohistochemistry. sh-Hiwi MFH clones $\mathrm{C}$ and $\mathrm{E}$ were induced with doxycycline for 3 days before IHC analysis.

9. Short-hairpin Hiwi knock down re-establishes differentiation capacity and reduces colony formation capacity

We then asked what the effect of Hiwi down-regulation might be on the capacity of MFH sarcoma cells to differentiate down a typical mesenchymal lineage. Although MFH cells do not undergo induced mesenchymal differentiation [9,93], dox-ind-sh-Hiwi MFH cells are able to undergo mesenchymal differentiation following doxycycline treatment and exposure to differentiation medium (Figure 2.9A). Here, osteogenic differentiation is shown with the Alizarin Red S stain, as previously described. In contrast, in the absence of doxycycline (parental MFH 
cells) rapidly overgrow and die (as previously described $[9,94]$ ), presumably because they do not respond to the differentiation signals that the dox-ind-sh-Hiwi-MFH cells are able to respond to.

A

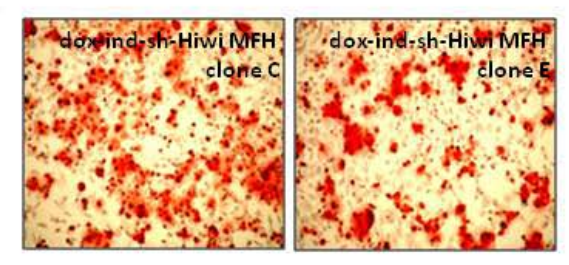

C

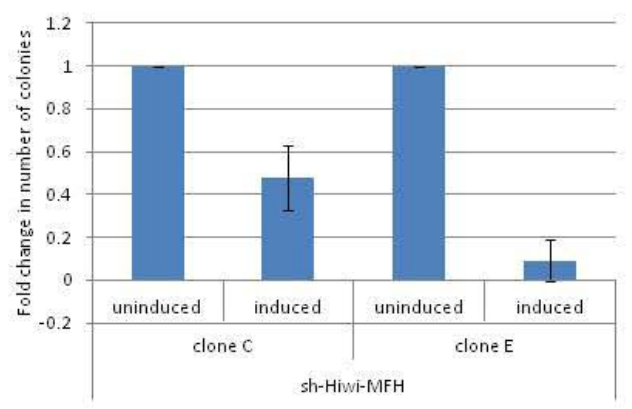

B
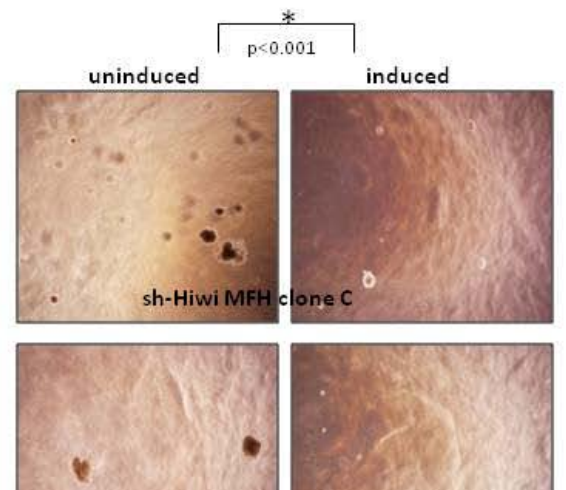

sh-Hiwi MFH clone E

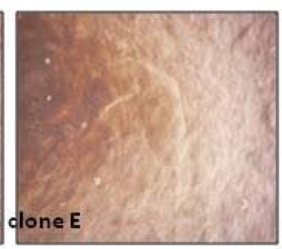

Figure 2.9 Hiwi knock down re-establishes differentiation and reduces colony formation of sarcoma cells

(A) Day 21 in osteogenic media. Calcium matrix formation (measured by Alizarin Red S stain) is restored in sh-Hiwi MFH clones $C$ and $E$ (approximately $50 \%$ of cells staining), induced with doxycycline 7 days before addition of differentiation media and continued in doxycycline-spiked media during differentiation. (B) Doxycycline-induced sh-Hiwi MFH cells at 4 weeks in colony forming assay show decreased colony formation, as compared to uninduced sh-Hiwi MFH cells. $p<0.001$ for clone $C$ and $p<0.005$ for clone E by Student's T Test. Representative pictures shown at 20X magnification (C) Quantification of colonies shown as fold change. Experiment was performed in quadruplicate and average quantification is shown. 
We then further characterized these Hiwi knock down MFH cells by examining their colony forming capacity. In vitro colony formation is a commonly used proxy for the tumorigenic potential of a given cell line. Colony formation is significantly reduced to about $40 \%$ of untreated control for dox-ind-sh-Hiwi MFH clone $\mathrm{C}$ and to about $15 \%$ of untreated control for dox-ind-shHiwi MFH clone $E$ ( $p<0.001$ for clone $C$ and $p<0.005$ for clone $E$; Figure 2.9B, quantified in Figure 2.9C).

\section{Discussion}

Using mesenchymal stem cells, transgenic mouse models and human tumor samples we show through this set of studies that Hiwi is directly tumorigenic, revealing a novel oncogenic role for Hiwi as a driver of tumorigenesis. We have identified increased Hiwi protein expression in undifferentiated human sarcomas, though a direct genetic basis for this up-regulation of Hiwi in sarcomas is still lacking. We found no characteristic chromosomal amplification of Hiwi in Hiwi-expressing human sarcomas. Moreover, we have generated in vitro Hiwi over-expression and Hiwi knock down models which indicate that Hiwi expression blocks mesenchymal differentiation capacity and transforms cells to become tumorigenic. Finally, an appendicular, mesoderm-specific transgenic mouse model of Hiwi shows that Hiwi over-expression forms sarcomas in vivo, albeit at a low rate of tumor incidence.

To date, Hiwi has been found to be highly expressed in a variety of human cancers, including gastric [69], gliomas [65], pancreatic [67] and squamous cell carcinomas [66]. The majority of reports which identify increased Hiwi expression in cancer focus predominantly on its clinical significance. For example, in sarcomas [26], hepatocellular carcinomas [95], and in colorectal cancer [96], Hiwi expression is an indicator of poor patient prognosis. Because the focus of previously published studies was the role of Hiwi as a prognostic factor for various types of cancer, the question of whether Hiwi was driving tumorigenesis or whether it was 
elevated secondarily in response to another driver of the tumorigenesis was not asked. None of previously published studies have focused on the investigation of a potential oncogenic role for Hiwi in driving tumor formation and/or maintenance. Our studies are the first to prove Hiwi can be a driver of sarcomagenesis and that sarcomas may be addicted to Hiwi expression for the maintenance of the tumor phenotype. This novel oncogenic role for Hiwi shows that, while it may also be an important prognostic factor, Hiwi may be a viable therapeutic target.

Throughout our short hairpin Hiwi knockdown studies in MFH sarcoma cells, we employ the commonly used technique of colony formation assays as an in vitro proxy for tumorigenic growth. Since xenograft experiments were used for exploring the tumorigenicity Hiwi overexpressing MSCs, we also attempted an analogous xenograft approach for our doxycyclineinducible sh-Hiwi MFH model. In vivo treatment of doxycycline to achieve continuous doxycycline-inducible gene expression changes in xenograft systems has been established [97]. We attempted a similar experiment, in which sh-Hiwi MFH cells were induced in vitro with doxycycline, then subcutaneously injected into SCID mice. To maintain knock down of Hiwi in vivo, the inoculated mice were given doxycycline water over the course of the experiment and tumor formation was monitored. We found no significant changes in tumorigenic growth of the subcutaneously injected dox-sh-Hiwi MFH cells, as compared to control sh-Hiwi MFH cells which were never induced with doxycycline (Figure 2.10A). Additionally, IHC staining for Hiwi in these xenografts shows no change in Hiwi expression during doxycycline induction in vivo (Figure 2.10B). Because our colony forming assay results directly oppose these in vivo data, we venture that perhaps the doxycycline in the drinking water is not reaching the inoculation sites. This might be due to a lack of xenograft vascularization soon after inoculation, preventing doxycycline from maintaining Hiwi knockdown. Once there is sufficient tumor vascularization, and doxycycline is able to reach the site, the tumorigenic growth has already occurred and doxycycline-induced Hiwi knock down occurs too late to affect tumorigenic growth. Rather than 
parsing out the best method for doxycline delivery, we focused instead on more reliable and consistent in vitro colony formation assays during doxycline-induced Hiwi knock down, in which continuous Hiwi knock down is much more straightforward to ensure. Colony formation assays have previously been reported to measure the tumorigenic growth of cancer cells $[98,99]$ and their set up contains doxycycline for continuous Hiwi knock down. Other commonly used in vitro assays for aspects of tumorigenicity are proliferation and invasion assays. However, our decision to use colony formation assays reflects the fact that colony formation assays already include aspects of cellular proliferation and invasion. Moreover, they are quantitative, providing for statistical analysis of the data. 
A

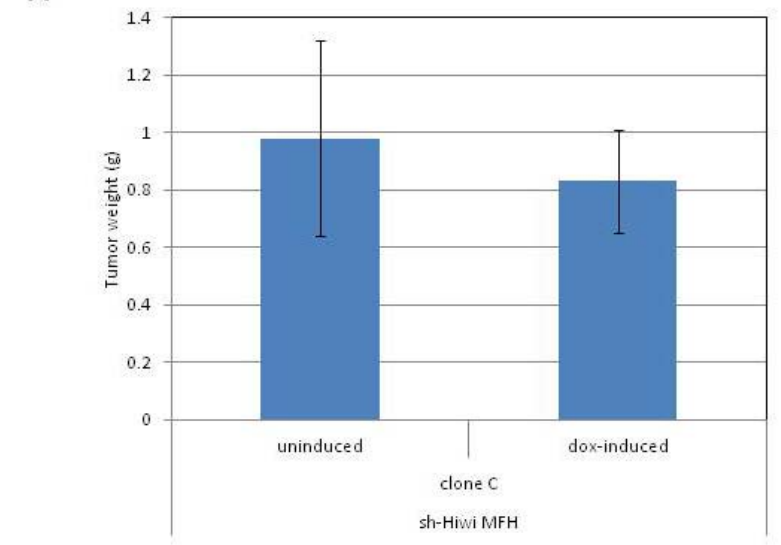

B

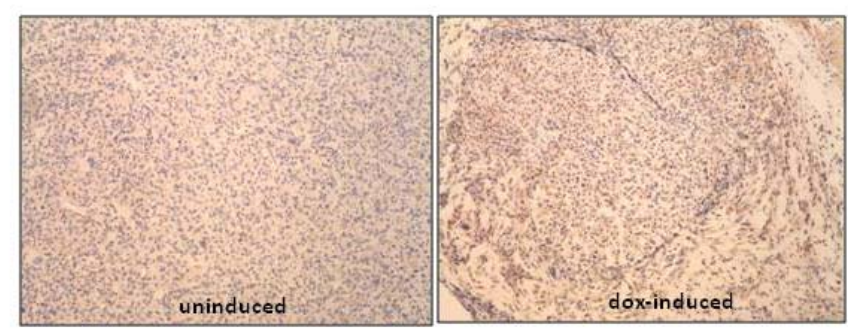

sh-Hiwi-MFH clone C

\section{Figure 2.10 sh-Hiwi MFH xenograft weights}

(A) Doxycycline induction in vivo does not affect sh-Hiwi MFH xenograft growth, likely due to difficulty in doxycycline arriving to the inoculation site to maintain Hiwi knockdown. For simplicity, only clone $\mathrm{C}$ shown, although similar results were obtained from all clones tested. Error bars represent standard error and xenografts were performed in duplicate. (B)Representative pictures of xenografts derived from clone $\mathrm{C}$ stained for Hiwi. All pictures are shown at $10 \mathrm{X}$ magnification.

Ours is also the first transgenic mouse model of Hiwi-mediated sarcoma formation.

Xenograft assays of Hiwi over-expressing MSCs suggest that Hiwi drives tumor formation.

Based on these data, we generated a mouse model of Hiwi over-expression under control of the

Prx1 promoter [91]. While the tumor incidence rate of these Prx-Hiwi mice is less than $10 \%$, it is still higher than that of transgenic mice in which the Retinoblastoma (Rb1) tumor suppressor gene is deleted under the same Prx1 promoter. These Rb1 data lead us to believe that the PrxHiwi transgenic mouse model does not fully recapitulate the tumorigenic potential of Hiwi. It is 
possible that Hiwi expression levels are simply too low under the Prx1 promoter to induce massive, widespread tumorigenic growth. To this end, we have begun work on a transgenic mouse model of Hiwi expression under a pROSA-driven floxed stop which is crossed with Prx1Cre mice to induce Hiwi expression in Prx1 tissues (limb bud mesoderm), only after Cre recombination. Another possibility for the low tumor incidence rate of the Prx1-Hiwi mouse model is that Hiwi over-expression alone is sufficient to establish tumors at a very low rate, but that Hiwi over-expression in a tumor-sensitive background leads to higher rates of tumor formation. Hiwi might be more robustly oncogenic when there is a concurrent loss of Rb or Pten tumor suppressor genes. To test this, we have begun crossing Prx1-Hiwi mice with those that have a Prx1-specific loss of either Rb or Pten tumor suppressors. This is further discussed in Chapter 5.

Though increased expression has been described in a variety of human cancers, the exact role of Hiwi in adult neoplastic tissues had not been explored. Taken together, the studies described here are the first report of a novel oncogenic role for Hiwi as a driver of sarcomagenesis. Using in vitro cell line models, a transgenic mouse model and human primary sarcoma samples, we show that Hiwi is highly expressed in primary sarcomas and that Hiwi expression results in both a differentiation block down typical mesenchymal lineages and increased tumorigenicity. 


\section{Materials and Methods}

\section{Colony Formation Assays}

Cells were suspended in $0.3 \%$ agar (Sigma) in culture medium and plated into 6 well plates, with a base layer of $0.6 \%$ agar in culture medium. For doxycyline and 5 -azacytidine treatments, cells were pre-treated for 7 days in culture before beginning colony formation assay. Cells were kept in drug-spiked media during the assay and monitored for colony formation. Pictures were taken after 4 weeks. Experiments were performed in 2 times, each time in duplicate.

\section{Immunohistochemistry (IHC)}

IHC was performed as previously described by us [93]. Briefly, formalin-fixed, paraffinembedded (FFPE) tissues were rehydrated and treated with citric buffer for antigen retrieval. Slides were blocked with $10 \%$ horse of goat serum in $2 \%$ BSA-PBS and then incubated in primary antibody (diluted in $2 \%$ BSA-PBS) overnight at $4^{\circ} \mathrm{C}$. Following 30 minutes of secondary antibody and tertiary antibody (Vector Labs) incubation, slides were developed with 3,3Diaminobenzidine (DAB) and counterstained with Hematoxyalin. For cells, fixation was performed with 50\% Methanol/Acetone for 10 minutes, then blocking, primary antibody incubation, and detection was performed as for FFPE IHC. Primary antibody used in these studies: Hiwi (Abcam, 12337)

\section{Sarcoma Tissue Microarray (TMA)}

The TMA contained 10 cases of each sarcoma subtype in triplicate. For TMA analysis, IHC staining was scored on a scale of 0 to 2 by multiple sarcoma pathologists. The average score for each sarcoma subtype was calculated and representative pictures are shown.

\section{Western Blotting}


Standard western blotting technique was used. Briefly, protein lysate was collected from cells with RIPA buffer (Boston BioProducts) and 50ug were run on a 4-20\% Tris-glycine gradient gel (Invitrogen). Nitrocellulose membranes were incubated overnight at $4^{\circ} \mathrm{C}$ in primary antibody: Hiwi (1:500 ProSci 45-735P).

\section{Bone and Fat Differentiation}

Bone or fat differentiation was assessed in MSCs after 21 days in bone or fat differentiation media, as previously published [9]. Approximate percentage of differentiated cells was calculated based on the average alizarin red s or oil red o staining over 3 independent experiments.

\section{Xenograft Generation}

NOD-SCID mice were subcutaneously injected, in triplicate, with 1 million cells of each indicated cell type, as described previously [93]. Tumor formation was monitored for 5 weeks and mice were sacrificed when tumor size reached $1 \mathrm{~cm}$, in accordance with Columbia University Animal Welfare and IUCAC policy under IRB protocol AAAA9669.

\section{Quantitative RT-PCR}

RNA was collected using Qiagen RNeasy kit. Complimentary DNA (cDNA) was made from 1ug of RNA, according to instructions in the SuperScript III cDNA synthesis kit (Invitrogen). 1uL of cDNA was used in Qiagen QuantiTect Sybr mix, according tom manufacturer's instructions. Samples were run on Stratagene MX3005p using the following cycling: 95 x 15m: 1 cycle /94 x 15s; 55 x 30s; 70 x 30s: 40 cycles/95 x 1m; 54 x 30s; 95 x 30s; 25 x 10s: 1 cycle. Hiwi primer 
sequence: F: CCCCAAGTCAGTGTGTGGTG, R: TATGCAGCGTGAGAACCAGC. Normalizing Actin primers: F: AAACTGGAACGGTGAAGGTG, R: GTGGCTTTTAGGATGGCAAG

\section{DNA Fluorescence In Situ Hybridization (DNA FISH)}

DNA FISH was performed by Dr. Murty and colleagues at the CUMC Cytogenetics Core Facility. A probe against the Hiwi locus on chromosome 12 was designed and used to identify chromosomal amplification of samples present on the sarcoma TMA. 


\section{Chapter 3: Identification of piwi-interacting RNAs (piRNAs) in}

\section{mesenchymal stem cells}

\section{piRNAs are present in germline and somatic cells}

A novel class of small RNAs, called piwi-interacting RNAs (piRNAs), maintains genome integrity by epigenetically silencing transposons via DNA methylation, especially in germline stem cells. piRNAs are thought to interact exclusively with the Piwi family of proteins.

A large focus of piRNA research has been dedicated to elucidating their role in germline stem cells. piRNAs were isolated from rodent testes by 4 different groups simultaneously $[54,56,57,70]$, suggesting that this novel class of small RNAs were specific to male germline stem cells. Additionally, the zebrafish Piwi homolog, Ziwi, is expressed exclusively in the gonad [71], further suggesting that the piRNA pathway is specific to the germline.

The germline restriction of piRNAs has recently been called into question by a growing body of evidence examining piRNAs in various cell types. piRNAs have been identified outside germ stem cells, for example, in somatic cells of the Drosophila ovary [78]. A recent study also found piRNAs in neurons of the mouse central nervous system [79]. The authors found that piRNA inhibition results in a decrease in dendritic spine formation, a key measure of synaptic function. Recent work identified piRNA-like molecules in various fly, mouse and macaque somatic tissues, including pancreas and cortex [80]. It is important to note that while these piRNA-like molecules were comparable to piRNAs in most relevant criteria (length, distribution) and Piwi family proteins were identified in the examined tissues, a direct association of these small RNAs with Piwi family proteins was not shown. Taken together, these data suggest that piRNA populations may exist in a variety of somatic tissues. 
A piRNA population has also been identified in HeLa cells [81], a cervical cancer-derived cell line. Although the piRNA population in HeLa cell is much smaller compared to that found in murine germline stem cells, it is still localized to the periphery of the nucleus, consistent with previous studies [82]. Moreover, Wu and colleagues recently identified a piRNA population in murine mesenchymal stem cells (MSCs) [83]. These piRNAs associate with Mili in the cytoplasm of MSCs and are thought to be important for keeping cellular proliferation in check. Importantly, Mili expression was examined by immunoprecipitation and western blot using the same Mili antibody, suggesting that actual Mili expression is much lower than the enriched band shown. There was no evidence that other murine Piwi orthologs are present in MSCs. Additionally we have shown that Mili, Miwi and Miwi2 (all 3 murine Piwi orthologs) are not readily detectable by RT-PCR from murine MSCs (Figure 3.1). Together, these data suggest that, while piRNAs may be present in a variety of stem cell and somatic cell types, Piwi protein expression is much more limited, as it is found only in the germline during normal development. 


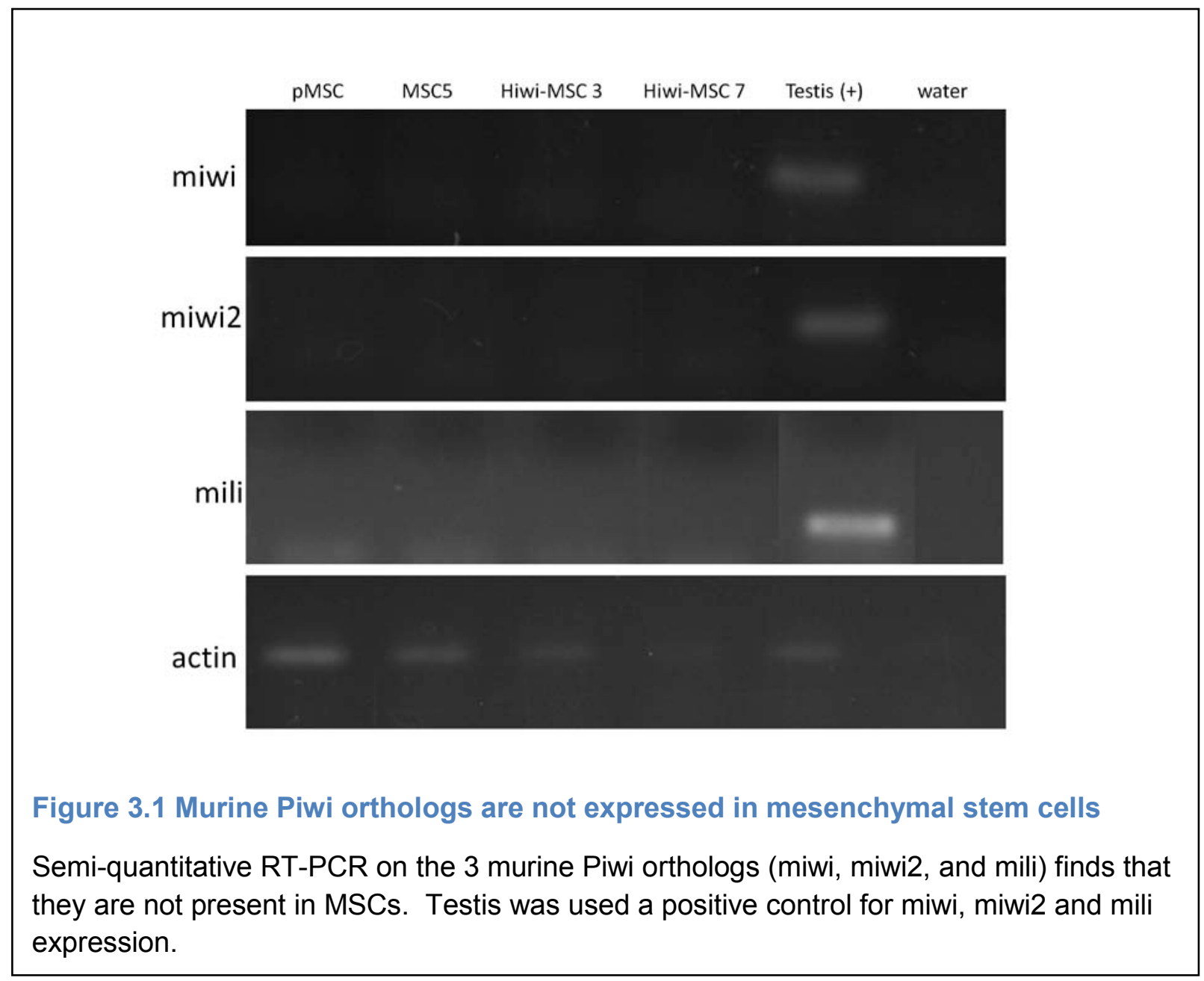

piRNAs are known to associate exclusively with Piwi proteins. Moreover, piRNAs rely on Piwi homologs for their biogenesis, via an alternate cleavage mechanism called the pingpong cycle $[74,100]$. In the ping pong cycle, there is cleavage of the 5 ' or 3 ' end of piRNA precursors via piRNA-Piwi complexes to generate functional piRNAs and silence transposon targets [36] . The data from a variety of labs which suggest piRNAs exist in a variety of germline and somatic tissues (described above) suggest that piRNAs may exist in cells which do not express Piwi homologs. This raises an interesting question: how are piRNAs generated in absence of Piwi proteins? It is possible that there is another as yet unidentified Piwi homolog in each of the tissues where piRNAs have been found. Alternatively, there may be non-ping pong cycle mechanisms for piRNA generation that do not require Piwis. 
Furthermore, piRNAs have recently been found in adult human brain and testes samples [101]. Intriguingly, a specific piRNA, piR_015520 has been identified in the human melatonin receptor $1 \mathrm{~A}$ gene and exogenous expression of this piRNA is able to down-regulate melatonin receptor $1 \mathrm{~A}$ gene transcript levels [101]. piR_015520 sits within intron 1 of the melatonin receptor $1 \mathrm{~A}$ gene and is a sense piRNA. This is the first report of piRNA-mediated regulation of a protein-coding gene, rather than a transposon repeat element. Much more work must be done to determine additional piRNAs which correspond to gene sites and regulate gene expression, but these data suggest that piRNAs may be regulating gene expression (not only transposon expression) outside the germline and in human cells, suggesting that piRNAs regulate gene expression more broadly that previously thought.

\section{Rationale of the Study}

Because previous studies identified piRNAs in germline cells which express high levels of Piwi family members during development, it is thought that this class of small RNAs interacts exclusively with Piwis to exert their gene silencing effects. Recently piRNAs have been identified in a variety of somatic tissues as well. We asked whether piRNAs might also be present in our Hiwi over-expressing MSCs, although the parental MSCs do not express any mouse Piwi orthologs. If piRNAs are present, we further queried if the populations would be different in Hiwi expressing MSCs (MSC-Hiwi 3 and MSC-Hiwi 7) compared to Hiwi nonexpressing MSCs (parental MSCs and MSC5). Lastly, we asked whether a potential piRNA population would correspond to genic regions, transposon repeats or other genomic locations.

\section{Experimental Design}

In order to test for the presence of piRNAs in our Hiwi over-expressing we performed SOLiD sequencing on the entire small RNA population in our parental MSCs and MSC5 (do not express Hiwi) and in MSC-Hiwi 3 and MSC-Hiwi 7 (over-express Hiwi). Known microRNAs 
(miRNAs) were filtered out, as were all small RNAs outside the known size range of piRNAs. The remaining piRNA reads were referenced against the piRNA bank database, although that contains only germline-derived piRNAs, to identify known piRNAs. Novel putative piRNAs which met all other criteria but were not present in piRNA bank were also included in the analysis. All piRNAs were then examined for genomic location.

\section{Results}

\section{Small RNA sequencing reveals presence of piRNAs in mesenchymal stem cells}

piRNAs are expected to be present in tissues where Piwis are also present. Our expectation was that piRNA would be present in the MSC-Hiwi 3 and 7 Hiwi over-expressors. Therefore, we were surprised to find that a piRNA population is present in both the MSC-Hiwi 3 and 7 Hiwi over-expressors, but also in parental MSCs and MSC5 which do not express Hiwi. This suggests that a piRNA population may exist in a variety of tissues, including somatic tissues. Moreover, the presence of Hiwi is not necessary for the existence of piRNAs.

\section{2. piRNA expression patterns do not shift in Hiwi-expressing cells}

After identifying the presence of piRNAs in parental MSCs and in Hiwi-expressing MSCs, we asked whether piRNAs are differentially expressed in Hiwi expressing MSCs compared to parental MSCs. Previously, Kuramochi-Miagawa and colleagues [45] identified a characteristic shift in the piRNA population found in either wildtype or Mili/Miwi2 knockout mouse testes. In order to test whether our Hiwi-MSCs show a characteristic shift in piRNA expression pattern, we compared the piRNAs in each of the MSC-Hiwi over-expressors with each of the Hiwi nonexpressing MSCs (Figure 3.2 A-D). There are a few piRNAs which are highly expressed in Hiwi expressing MSCs compared to Hiwi non-expressing MSCs (Figure 3.2 A-D, above the top red line). However, there is a similar number of piRNAs which are highly expressed in the Hiwi non-expressing MSCs compared to Hiwi expressing MSCs (Figure 3.2 A-D, below the bottom 
red line). Moreover, the majority of piRNAs lie along the line $\mathrm{x}=\mathrm{y}$ (within the red lines), suggesting that the piRNA expression patterns don't shift significantly when Hiwi is overexpressed. Taken together, these data suggest that there is no characteristic shift in the piRNA populations in Hiwi expressing MSCs versus Hiwi negative MSCs; rather a few piRNAs are expressed more highly in the presence of Hiwi and others are expressed at lower levels. There seems to be a shift in piRNAs in both directions in Hiwi-MSCs, though not in significant numbers.
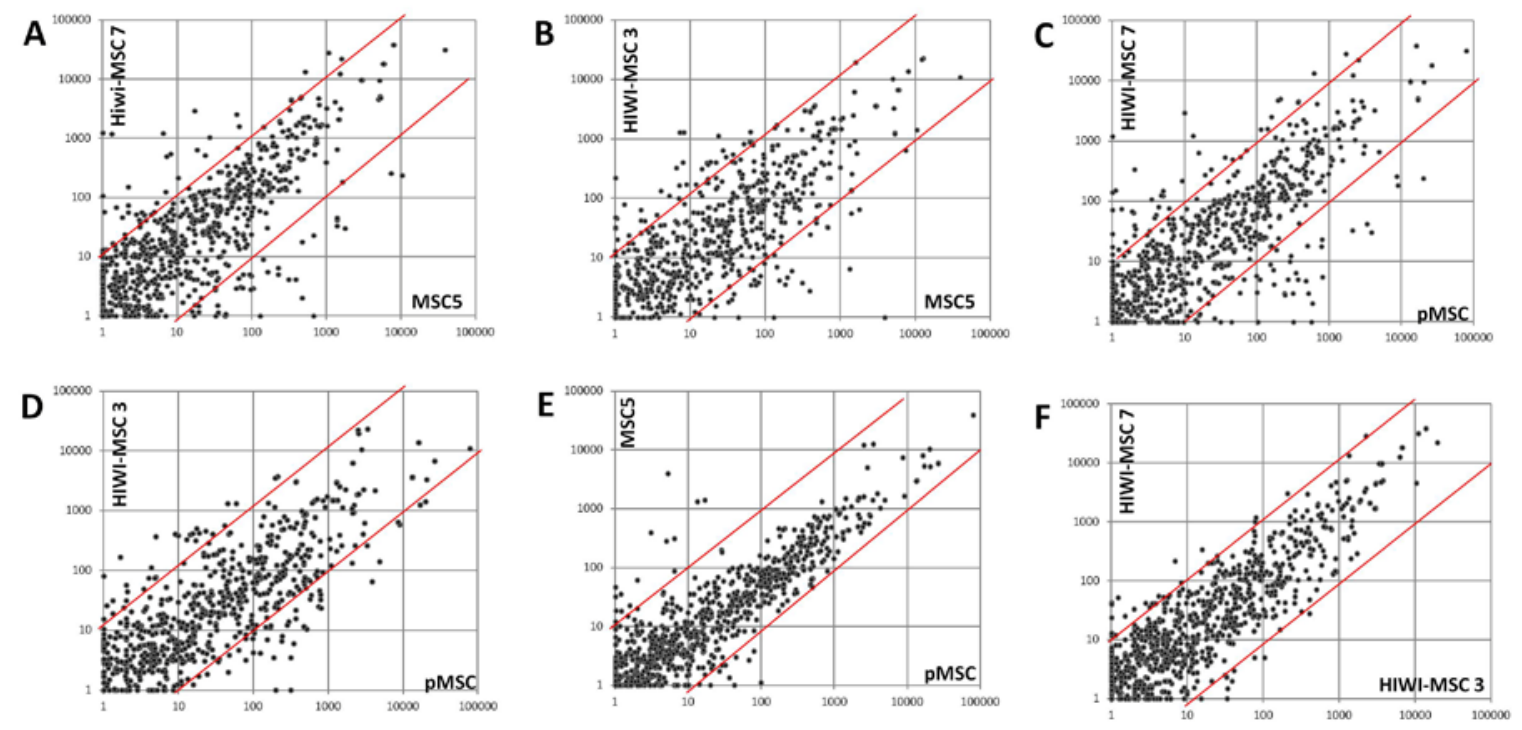

Figure 3.2 piRNA expression patterns do not shift in Hiwi-expressing cells

(A-D) Comparison of the piRNA expression pattern in a Hiwi positive sample to that of a Hiwi negative sample. (E) Comparison of the piRNA expression pattern in both Hiwi negative samples. (F) Comparison of the piRNA expression pattern in both Hiwi positive samples. Red lines mark the line $y=x$; points outside the red lines represent differentiallyexpressed piRNAs.

We then asked if the few differentially expressed piRNAs that we see (in both Hiwi expressing and Hiwi non-expressing MSCs) are true changes in piRNA expression or if they are due to clonal variation. To test this, we compared both Hiwi negative MSCs to each other 
(Figure 3.2E) and both Hiwi expressing MSCs to each other (Figure 3.2F). In these comparisons we find the vast majority of piRNAs are not differentially expressed (they sit inside the red lines). There are a few piRNAs that sit outside the red lines, but these are very few compared to any of the Hiwi expressing MSC versus Hiwi non-expressing MSC analyses. This suggests to us that the few differentially expressed piRNAs seen in Figure 3.2E, F are due simply to clonal variation rather than Hiwi expression.

Our data indicate that, although there are a few piRNAs that are differentially expressed in the presence of Hiwi, there are just as many that are differentially expressed in Hiwi negative MSCs. There is no characteristic shift in the piRNA expression patterns in the presence or absence of Hiwi.

\section{3. piRNAs in mesenchymal stem cells do not correlate to gene sites}

piRNAs in the germline are known to silence transposon repeat regions in order to maintain genome integrity. However, a recent study has identified a piRNA which regulated expression of a protein-coding gene, rather than a non-genic repeat region [101]. To identify any gene

\begin{tabular}{|c|c|c|c|c|c|c|}
\hline piRNA location & Gene & $\begin{array}{c}\text { piRNA } \\
\text { expression in } \\
\text { Hiwi+ }\end{array}$ & $\begin{array}{c}\text { Gene } \\
\text { expression in } \\
\text { Hiwi+ }\end{array}$ & $\begin{array}{c}\text { Gene } \\
\text { expression in } \\
\text { Hiwi KD }\end{array}$ & $\begin{array}{c}\text { piRNA } \\
\text { relationship } \\
\text { with Hiwi }\end{array}$ & Strand \\
\hline $\begin{array}{c}\text { chr11+ } \\
54288915\end{array}$ & Fnip1 & high & low & high & direct & plus \\
$\begin{array}{c}54288990 \\
\text { chr16+ } \\
43168305 \\
43168395\end{array}$ & Zbtb20 & low & low & high & inverse & plus \\
\hline
\end{tabular}

Figure 3.3 Only two identified piRNA reads correspond to genes

Two piRNAs found in Hiwi-MSCs correspond to genes. One has a direct relationship with Hiwi and one has an inverse relationship with Hiwi. 
regions to which piRNAs in our Hiwi-MSCs might correspond, we performed a bioinformatic analysis of the piRNAs and the mouse genome. We found that there vast majority of piRNA did not correspond to gene loci. There were only2 identified piRNA reads which correspond to genes (Figure 3.3). These 2 genes are Fnip1 and Zbtb20. The piRNA corresponding to Fnip1 is upregulated in Hiwi-expressing MSCs and the Fnip1 gene itself is down-regulated in Hiwiexpressing MSCs, suggesting a direct relationship of this piRNA with Hiwi. On the other hand, the piRNA corresponding to Zbtb20 is expressed at low levels in Hiwi-expressing MSCs and the gene itself is also expressed at low levels in Hiwi-expressing MSCs, suggesting an inverse relationship of this piRNA with Hiwi. Because these 2 piRNA-Hiwi relationships are opposing, and because the vast majority of piRNAs do not correspond to gene regions, we interpret these data to suggest that piRNAs likely do not generally regulate gene expression in our Hiwi-MSCs. Moreover, many piRNAs we identified correspond to repeat regions, though not directly to transposons. The majority of piRNAs identified do not correspond to any known genome elements.

\section{4. piRNAs in mesenchymal stem cells do not generally lie upstream of gene sites}

After multiple analyses, we identified only 2 piRNAs which corresponded to potential target genes. Since this approach identified such a small segment of the piRNA population, it is possible that these 2 piRNAs were identified by chance in our bioinformatic analyses. To account for that, we broadened our analysis of differentially expressed piRNAs to include those that lie within 2 or 10 kilobases (kb) of transcriptional start sites (TSS) of potential target genes. Even with this relatively loose threshold, we identified only 5 additional piRNA reads that lie 2 or $10 \mathrm{~kb}$ upstream of target genes (Figure 3.4). Of these 5 piRNAs, only 1 sits within $2 \mathrm{~kb}$ upstream of a gene: Mfsd11, which has an unknown function. 


\begin{tabular}{|c|c|c|c|c|}
\hline $\begin{array}{c}\text { piRNA distance } \\
\text { from TSS }\end{array}$ & Gene & $\begin{array}{c}\text { piRNA expression } \\
\text { in Hiwi+ }\end{array}$ & $\begin{array}{c}\text { Gene expression } \\
\text { in Hiwi+ }\end{array}$ & $\begin{array}{c}\text { piRNA } \\
\text { relationship } \\
\text { with Hiwi }\end{array}$ \\
\hline $2 \mathrm{~kb}$ & Mfsd11 & low & high & inverse \\
\hline $10 \mathrm{~kb}$ & Acsl1 & low & high & inverse \\
\hline $10 \mathrm{~kb}$ & Samd8 & low & high & inverse \\
\hline $10 \mathrm{~kb}$ & Gabbr1 & high & low & direct \\
\hline $10 \mathrm{~kb}$ & Xpo7 & high & low & direct \\
\hline
\end{tabular}

Figure 3.42 or $10 \mathrm{~kb}$ upstream analysis reveals 5 piRNAs that lie upstream of genes

5 piRNAs were found either $2 \mathrm{~kb}$ ( 1 piRNA) or $10 \mathrm{~kb}$ ( 4 piRNAs) upstream of TSS are identified. 3 have an inverse relationship with Hiwi and 2 have a direct relationship with Hiwi.

This piRNA is decreased in Hiwi-MSCs compared to parental MSCs and Mfsd11 is increased in Hiwi-MSCs, suggesting an inverse relationship of this piRNA with Hiwi. We had to expand our analysis even farther, to $10 \mathrm{~kb}$ upstream of TSS, in order to identify 4 additional piRNAs. Of these 4, 2 have an inverse relationship with Hiwi and 2 have a direct relationship with Hiwi. These 4 genes (Acsl1, Samd8, Gabbr1, Xpo7) have diverse roles (see Discussion). Taken together, the data from this less stringent analysis give only 5 additional piRNAs and target genes. Moreover, the 5 piRNAs identified in this less stringent analysis reveal opposing piRNA-Hiwi relationships. The vast majority of piRNAs, even examining up to $10 \mathrm{~kb}$ upstream, do not correspond to gene regions.

\section{Discussion}

In this set of studies, we first asked whether piRNAs are present in our Hiwi-MSCs. To our surprise, piRNAs were found, not only in Hiwi over-expressing MSCs (Hiwi-MSC 3 and 7), 
but also in Hiwi non-expressing parental MSCs and MSC5. While piRNAs have been most wellcharacterized in germline cells during normal development, they have also recently been discovered in tissues outside the germline, including various fly and mouse tissues and in HeLa cells $[79,80]$. Our identification of piRNAs in MSCs is in line with these recent reports. Importantly, there is only one report of a piRNA which regulates a gene, rather than a transposon repeat region [101]. Similarly, we identified only 2 piRNAs which correspond to gene regions. These 2 genes are Fnip1 and Zbtb20. Fnip1 is a known folliculin-binding protein which has been associated with AMPK in the mTOR pathway $[54,102]$ thought to be important for nutrient sensing. However there is no direct evidence for any role it might play in cancer initiation or progression. Zbtb20 is a transcriptional repressor, especially active in the fetal mouse liver [103]. A transgenic mouse model for Zbtb20 knock out shows metabolic dysfunction and defective hippocampus development $[104,105]$. There is no indication of tumorigenic growth in this mouse model of Zbtb20 depletion. One recent study found that Zbtb20 expression is increased in hepatocellular carcinoma, and this correlates with a worse clinical prognosis [106]. However, in our tumorigenic Hiwi-expressing MSCs, Zbtb20 gene expression is decreased, suggesting that Zbtb20 (or its cognate piRNA) does not play a role in the Hiwi-mediated sarcomagenesis described in Chapter 2. Upon expansion of our analysis to identify piRNAs within $2 \mathrm{~kb}$ of a gene's TSS, we identified only one piRNA, whose potential target gene is Mfsd11. This gene has no known function. Further analysis of piRNAs within $10 \mathrm{~kb}$ of a gene's TSS reveals 4 more piRNAs which lie within $10 \mathrm{~kb}$ of 4 genes: Acsl1, Samd8, Gabbr1, Xpo7. These 4 genes have diverse functions, though not all have been implicated in cancer. Acsl1 plays a role in fatty acid degradation [107] but has not been shown to be involved in the progression of cancers. Samd8 has no known function. Gabbr1 is a Gammaaminobutyric acid (GABA) receptor subunit involved in synaptic transmission in the central nervous system [108]. One recent descriptive report suggests that Gabbr1 expression is 
decreased in nasopharyngeal carcinoma [109]. Gabbr1 is also decreased in our Hiwi-MSCs; however, there are no other reports of Gabrr1 in other cancers. Xpo7 is an export protein for molecules through the nuclear pore complex [110]. Importantly, there have been no studies implicating Xpo7 in cancer. Taken together, the data from our bioinformatic analyses reveal that very few piRNAs correspond to gene sites, even when regions up to $10 \mathrm{~kb}$ upstream of gene sites are examined. Moreover, the few piRNAs that were identified had opposing relationships with Hiwi and their potential target genes are generally not known to be involved in tumorigenic processes.

Other groups have used a variety of methodologies to identify piRNAs in their systems, especially in the male germline. Commonly, an RNA-immunoprecipitation (RNA-IP) approach is used to extract a piRNA population. In this technique, an antibody against a Piwi protein is used to capture the Piwi and its associated bound small RNAs. The small RNAs are then run on a gel to identify a piRNA population. While some groups have had success with this approach, there are some drawbacks which made it less desirable for our studies. Firstly, an RNA-IP approach requires an IP-quality antibody against the relevant Piwi protein. There are currently no commercially available IP-grade antibodies against Hiwi. We have found commercially available antibodies against Hiwi suitable for western blotting and IHC only. Secondly, an RNA-IP approach requires that Piwi proteins are present in the tissue being examined for piRNAs. Historically, piRNAs have been found in tissues which express Piwi proteins (ie, male germline). However, our studies suggest that a piRNA population exists in MSCs, even though no Piwi proteins are expressed in those cells. These data would have been missed with an RNA-IP approach. Thirdly, our sequencing approach generates detailed sequence data which can be checked against reference genomes with relative ease. We are utilizing an alternative experimental approach to further investigate the potential piRNA population in MSCs. We are using a tagged Hiwi vector to transfect cell lines of interest. This tagging approach is versatile, 
in that it circumvents the need for an IP-grade antibody against Hiwi and also that the overexpression of tagged Hiwi can be used to identify not only Hiwi-associated small RNAs, but also any Hiwi-associated proteins.

Our evidence that piRNA populations exist in both Hiwi expressing and Hiwi nonexpressing MSCs is surprising, but joins a growing body of evidence which finds piRNAs in tissues outside the male germline. However, these data raise an interesting question as to the origin of piRNAs in MSCs (as we show) and in other tissues where Piwis are not present (as others have shown). Because piRNA biogenesis is thought to occur via an amplification loop in which piRNA precursors associate with Piwi-piRNA complexes to be alternately cleaved at with 5 ' or $3^{\prime}$ ends, it is puzzling that a piRNA can exist in a cell without Piwi homologs. Our data suggest that piRNAs do exist in the absence of Piwis, and others have shown piRNAs in other cell types as well. Perhaps there is a still unidentified Piwi homolog present that can account for the presence of these piRNAs. Alternatively, there may be a non-ping pong-related mechanism for piRNA biogenesis. Our data and that of other groups identifying piRNAs in a variety of cell types open up a new set of questions in which piRNA origins may have to be reevaluated and further investigated. No matter how piRNAs are generated in the absence of Piwis, it seems that piRNAs are much more widespread that Piwi expression. This suggests that although piRNAs are present in a variety of cell types, they are only able to exert their silencing effects where Piwis are also present (in the germline during normal development and in cancer during aberrant tumor development). Moreover, our data indicate that there is no characteristic shift in piRNA expression patterns between the Hiwi expressing and Hiwi non-expressing MSCs. Lastly, we have found that piRNAs in MSCs generally do not correlate to gene regions. Taken together, these data suggest that while piRNAs are present in MSCs, they do not shift in response to Hiwi expression, nor do they regulate gene expression directly. 


\section{Materials and Methods}

\section{Semi-quantitative RT-PCR}

RNA was extracted from the indicated cell lines or from adult mouse testis tissue, using the RNeasy kit (Qiagen). $1 \mu \mathrm{g}$ of RNA was transcribed into cDNA using Super-Script III First Strand Synthesis System for RT-PCR (Invitrogen). To assess the expression levels of miwi, miwi2, mili and $\beta$-actin control semi-quantitative RT-PCR reactions containing Platinum Blue PCR mix (Invitrogen), $1 \mathrm{ul} \mathrm{cDNA}$ and corresponding primers were run at the following PCR program: $95^{\circ} \mathrm{C}$ x $2 \mathrm{~min} ; 95^{\circ} \mathrm{C} \times 30 \mathrm{~s}, 55^{\circ} \mathrm{C} \times 30 \mathrm{~s}, 68^{\circ} \mathrm{C}$ x $45 \mathrm{~s}$ for 28 cycles; $68 \times 5 \mathrm{~min}$. Experiments were performed in triplicate. Primer sequences were as follows: miwi: F:

TCGCAGGATTCGTCGCCAGC, R: CCCGCGGTCCTGAAAGACGC; Miwi2: F:

TTGTCCCTGTGGCCTGGGTTTG, R: AGAACGGTCTCATTGCGGAGGAC; mili: F: GCAAGGCTCCGGCACAGTCC, R: GTGGGCCCCCTGAACAACGG.

\section{Small RNA sequencing}

Small RNA sequencing was performed by Dr. Palomero and colleagues at the CUMC Genomics Core Facility. RNA samples were PCR-amplified with barcode tags. These amplicons were run on the SOLiD 3+ platform for sequencing. Initial bioinformatic analysis was performed by Dr. Pavel Sumazin (CUMC). 


\section{Chapter 4: Characterization of Hiwi-dependent epigenetic}

\section{mechanism of tumorigenesis}

\section{Piwis and DNA methylation}

In all model systems examined, Piwi family members are expressed in stem cells, including germ and hematopoietic stem cells, and are essential for germ line and/or somatic stem cell self-renewal $[2,28,29,30]$. Although the exact mechanism is still unclear even in the most well-studied models (e.g., drosophila, mice $[2,28,29,30]$ ), Piwi appears to ensure stem cell maintenance by inhibiting transposon migration $[36,58]$ during early development via an indirect (since Hiwi has no known direct chromatin modifying function) increase in DNA

methylation[55,59,60]. Specifically, previous studies have shown that transposon-specific DNAmethylation was reduced and transposon activity was elevated following silencing of Hiwi (or its homologs). Although transposons promote evolutionary diversity in lower organisms, their unchecked migration in higher organisms can result in disruption of genomic integrity [61] and thus Piwi proteins may have developed as an evolutionary defense system for multi-cellular species [58]. However, neither the precise epigenetic mechanism which leads to DNA methylation-based silencing, nor the exact epigenetic silencing machinery which may be involved in this increase in of DNA methylation have been explored.

\section{Rationale of the Study}

Because Piwi family members in well-studied model systems (Drosophila, mice) are known to silence transposons via DNA methylation-based mechanisms, we asked if this might also apply to our Hiwi over-expressing MSCs and our sh-Hiwi-MFH knock down cells. If DNA methylation is the mechanism by which Hiwi-expressing cells mediate sarcomagenesis, we further queried if treatment with a DNA de-methylating agent would be able to reverse Hiwi's effects. 


\section{Experimental Design}

To address a DNA methylation-based mechanism for Hiwi-mediated sarcomagenesis, we first assessed the global DNA methylation levels and expression levels of common transposon elements in our Hiwi MSCs. We then performed a promoter methylation array to examine which promoters specifically might be altered by Hiwi-dependent DNA methylation. We also assayed for changes in the expression of DNA methyltransferase proteins in our HiwiMSCs via western blotting. Next, we asked whether treatment with a DNA de-methylating agent, 5-azacytidine, is able to reduce the colony forming capacity of Hiwi-expressing MFH sarcoma cells. We also performed gene expression analyses of dox-ind-sh-Hiwi-MFH Hiwi knock down and 5-aza-cytidine treatment to see if they may be similar in terms of gene expression changes. Lastly, we performed an in silico analysis of 3 gene expression profile sets (Hiwi-MSCs, sh-Hiwi-MFH knockdown and 5-aza-cytidine treatment) in order to identify a specific tumor suppressor gene which is aberrantly silenced in our model system. Lastly, we return to our human primary sarcoma TMA and show that tumor suppressor expression in inversely correlated with Hiwi expression.

\section{Results}

\section{Transposon expression is decreased in Hiwi-expressing MSCs}

Based on data from a variety of model systems which find that Piwi mediates stem cell maintenance via DNA methylation, we decided to formally explore the DNA methylation status of our cells. Since Piwis are known to silence transposon expression, we examined the expression of 2 common transposon elements, IAP and Line 1 in our Hiwi-MSCs (Figure 4.1). We found that while there is some baseline expression of IAP and Line 1 transposons in parental MSC and MSC5, this is dramatically reduced in Hiwi-MSC 3 and Hiwi-MSC 7, which over-express Hiwi. Transposon expression is often used as a surrogate for global DNA 
methylation levels and these data would suggest that global DNA methylation levels decrease when Hiwi is expressed.

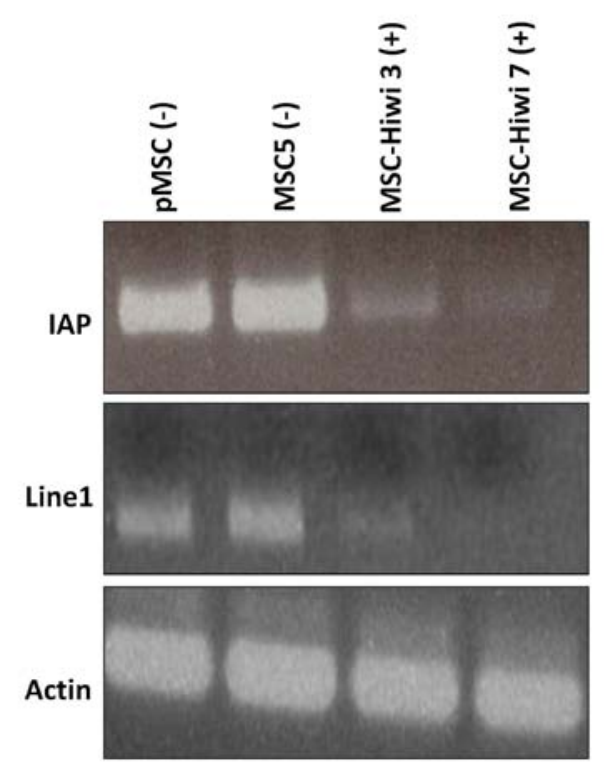

Figure 4.1 IAP and Line1 transposon expression decreases in Hiwi expressing MSCs

IAP and Line 1 semi-quantitative PCRs were run for 25 cycles. Actin is a loading control.

\section{Global DNA methylation correlates directly with Hiwi expression level}

We then decided to assay for global DNA methylation changes directly. Global DNA methylation was assessed by an ELISA-like assay, using an antibody against 5-Methyl Cytosine, which detects cytosine methylation at both $\mathrm{CpG}$ sites and non-CpG sites. Global DNA methylation was significantly increased (approximately 40\%, $p<0.05$; Figure 4.2A) in Hiwiexpressing MSCs (Hiwi-MSCs) as compared to non-Hiwi-expressing MSCs (pMSCs \& MSC5); suggesting that Hiwi expression leads to increase in DNA methylation. Similarly, we observe a 
significant decrease in global DNA-methylation (approximately 70\%, $p<0.05$; Figure 4.2B) during Hiwi down-regulation via doxycycline induction in sh-Hiwi-MFH.
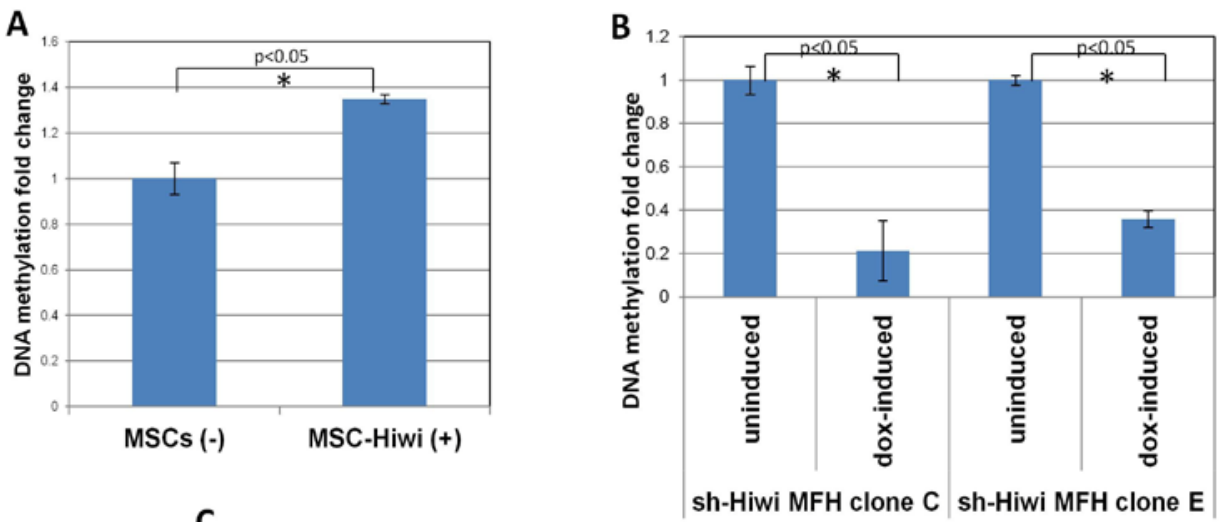

C

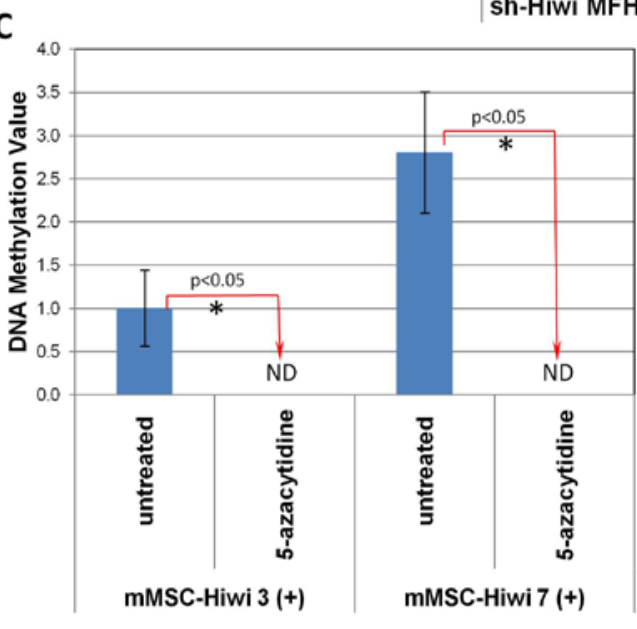

Figure 4.2 Global DNA methylation is increased in Hiwi-MSCs

(A) Global DNA methylation is increased in Hiwi-MSCs. Error bars represent standard error. * $=p<0.05$ by Student's T Test (B) Global DNA methylation is decreased in induced sh-Hiwi MFH cells. Cells were induced with doxycycline for 3 days before DNA was collected and assayed. Error bars represent standard error. ${ }^{*}=p<0.05$ by Student's $T$ Test (C) Global DNA methylation is decreased to non-detectable levels (ND) after $18 \mathrm{~h}$ treatment with 10uM 5-azacytidine in Hiwi-MSCs. Lower doses of 5-azacytidine (including $1 \mathrm{uM}$ ) did not change DNA methylation levels. Error bars represent standard error * $=p<0.05$ by Student's T Test

We then checked whether the Hiwi-mediated increase in global DNA methylation is reversible with the DNA-methyltransferase inhibitor, 5-azacytidine (Vidaza). Indeed, 5azacytidine treatment of Hiwi-MSCs completely $(100 \%, p<0.05)$ reverses the increase in global 
DNA-methylation mediated via Hiwi (Figure 4.2C) suggesting that DNMTs may be crucial intermediaries in Hiwi-mediated methylation. Of note, we did not observe any decrease in global DNA methylation following treatment of non-Hiwi-expressing MSCs with 5-azacytidine (data not shown), which may be reflective of the lower baseline global DNA methylation levels in those cells.

\section{DNA methyltransferase proteins are elevated in Hiwi-expressing MSCs}

Having established a direct correlation between Hiwi, DNA methylation, and Hiwi-associated DNA methylation reversibility via DNA-methyltransferase inhibitors, we next examined the levels of proteins known to modulate DNA methylation: DNA methyltransferase 1 (DNMT1), DNA methyltransferase 3 (DNMT3a), and methyl-binding protein 2 (MBD2). All three were found to increase in the presence of Hiwi (Figure 4.3). Interestingly, we find no expression of DNMT3b in Hiwi-MSCs. Our data are the first to show that expression of DNMTs correlates with Hiwi

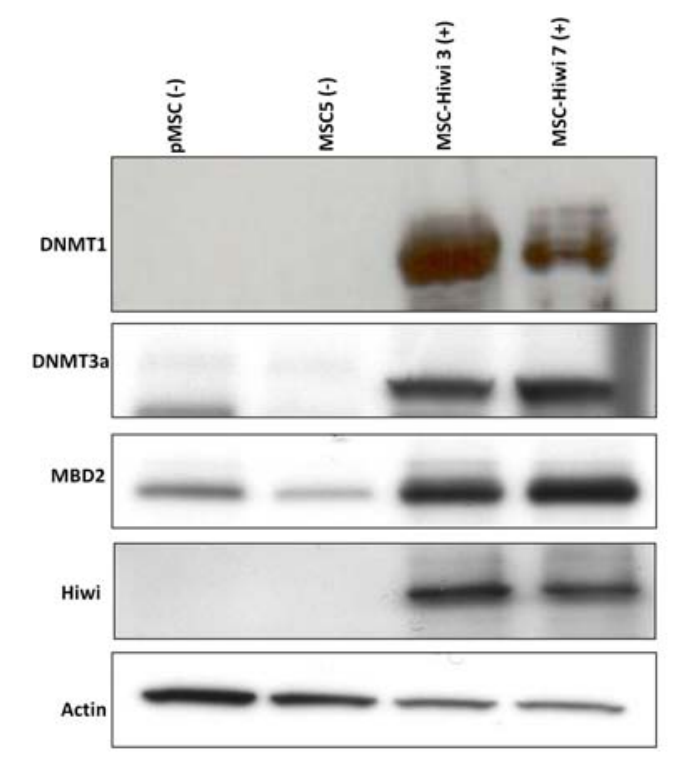

Figure 4.3 DNA methyltransferase proteins are elevated in Hiwi-MSCs

Western blots of indicated proteins reveal increased expression of DNMT1, DNMT3a and MBP2 in Hiwi-MSCs. Actin is a loading control. 
expression, providing a potential explanation of the increased DNA methylation found in Hiwiexpressing MSCs. However, the exact mechanism by which Hiwi expression leads to an increase in DNMT expression is still unclear. Moreover, we are unable to glean any further hint of mechanistic insight from developmental studies of Piwi proteins (which are much more thoroughly investigated) since the mechanism of Piwi is unknown in those developmental models $[2,28,29,30,36,55,58,59,60]$.

\section{5-azacytidine treatment restores transposon expression in Hiwi-expressing MSCs}

Since we have we shown that treatment of Hiwi-MSCs with 5-azacytidine can decrease global DNA methylation levels (Figure 4.2), we then asked if 5-azacytidine treatment is able to reverse the Hiwi-dependent transposon silencing we have previously observed (Figure 4.1). 5azacytidine is a DNA de-methylating agent which has been clinically approved for the treatment of myelodysplastic syndrome and acute myeloid leukemia [111] and is currently in clinical trials for a variety of disorders, reviewed in [112], We found that indeed 5-azacytidine treatment can reverse Hiwi-mediated transposon silencing (Figure 4.4A), restoring IAP and Line1 transposon expression back to levels comparable with parental MSCs as the concentration of 5-azacytidine increases. No such changes in transposons were observed in non-Hiwi expressing cells, pMSCs and MSC5. We further show that treatment of Hiwi-MSCs with either 5-azacytidine or 5aza-2-deoxycytidine can reverse Hiwi-mediated transposon silencing (Figure 4.4B, C). 5azacytidine treatments at lower concentrations (5uM or 10uM) show some restoration of Line1 transposon expression (Figure 4.4B, red box). However, a higher concentration of 5azacytidine treatment $(50 \mathrm{uM})$ is able to restore both IAP and Line1 transposon expression to levels comparable with parental MSCs, best seen for MSC-Hiwi7 (Figure 4.4C, red box) which has higher baseline Hiwi expression (Chapter 2) and is more tumorigenic (Chapter 2). No such changes in transposons were observed in non-Hiwi expressing cells (Figure 4.4B,C, left sided lanes). Treatment of Hiwi-MSC3 with both 5-azacytidine and 5-aza-2-deoxycytidine at 
50uM was highly toxic to the cells, despite our repeated treatment attempts. Taken together, these data suggest that treatment with a DNA demethylating agent is able to restore transposon expression. 

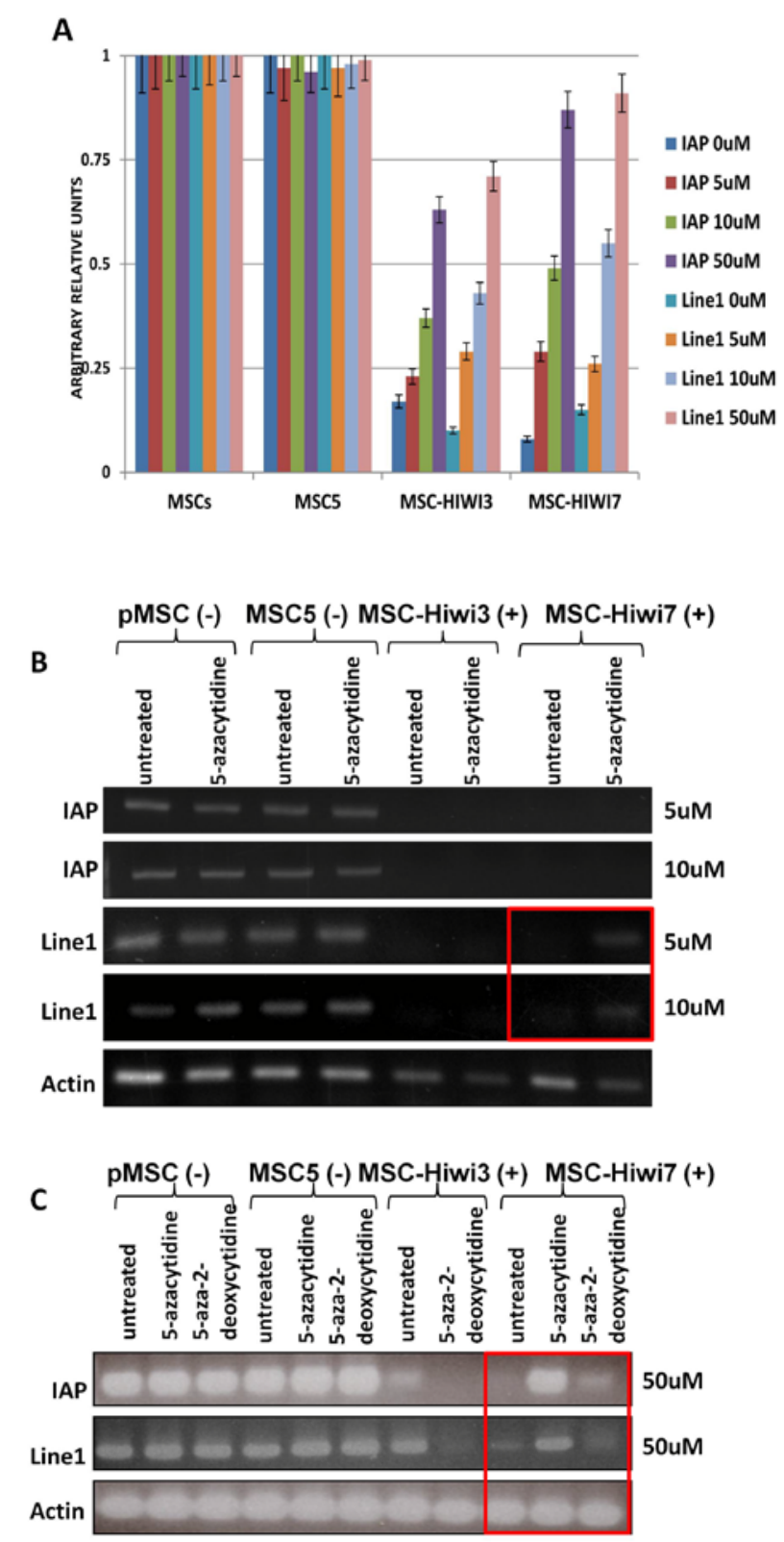

Figure 4.4 5-azacytidine treatment restores transposon expression in Hiwi-MSCs in a dose-dependent manner

(A) Treatment of Hiwi expressing MSCs with 0uM, 5uM, 10uM, or 50uM 5-azacytidine for $18 \mathrm{~h}$ followed by IAP and Line1 quantitative RT-PCRs. Parental MSCs of each 5azacytidine concentration were used to normalize the rest of the samples at that concentration. Error bars represent standard error. (B) Semi-quantitative RT-PCR for Line1 and IAP transposon expression on Hiwi-MSCs treated 18h with the indicated concentration of 5-azacytidine. Actin is a loading control. (C) Semi-quantitative RT-PCR for Line1 and IAP transposon expression on Hiwi-MSCs treated 18h with 50uM of 5azacytidine or 5-aza-2-deoxycytidine. Actin is a loading control. All experiments were performed in triplicate. 


\section{5-azacytidine treatment reduces colony forming capacity of sarcoma cells}

We have shown that treatment with a DNA de-methylating agent, 5-azacytidine, is able to reverse Hiwi-dependent transposon silencing. We then asked if 5-azacytidine treatment can reverse another Hiwi-dependent cellular effect: the colony forming capacity of MFH sarcoma cells. Indeed, 5-azacytidine treatment of sh-Hiwi MFH cells (in the absence of doxycycline) results in elimination of colony formation (Figure 4.5A), similar to that seen in doxycyclineinduced sh-Hiwi MFH (Chapter 2). Importantly, colony formation was significantly reduced, even at a $1 \mathrm{uM}$ dose of 5-azacytidine. Although higher doses were tested (data not shown), here we show the lowest effective dose. Colony formation during 5-azacytidine treatment was reduced to about $50 \%$ of untreated control for both sh-Hiwi-MFH clone $\mathrm{C}$ and sh-Hiwi MFH clone $E$ (combined $p<0.01$ for both clones) (Figure 4.5B). Of note, we previously published on the general insensitivity of sarcoma cell lines to 5-azacytidine treatment [113], but now suggest that Hiwi expressing tumors may be an exception. Taken together these results indicate that Hiwi is necessary for maintenance of the tumorigenic phenotype of Hiwi-expressing cells. 
A

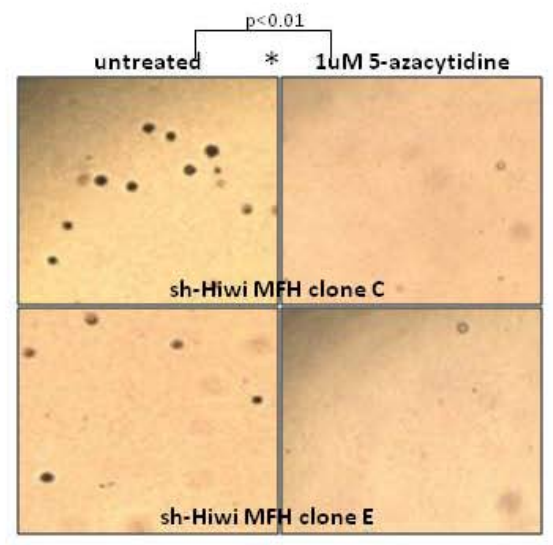

B

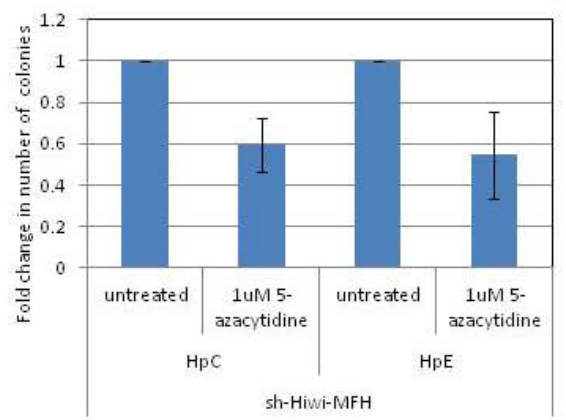

Figure 4.5 5-azacytidine treatment reduces colony forming capacity of sarcoma cells

(A) Untreated sh-Hiwi MFH cells or treated with $1 \mathrm{uM} 5$-azacytidine at 4 weeks in colony forming assay. 5-azacytidine treatment decreases colony formation capacity. Combined $p<0.01$ by Student's T Test. All experiments were performed in triplicate. Representative pictures shown at 20X magnification. (B) Quantification of colonies formed in either untreated sh-Hiwi-MFH or treated for 4 weeks with 1 uM 5-azacytidine. Averages of 4 experiments shown here. Error bars represent standard error.

\section{Gene expression changes during 5-azacytidine treatment and during Hiwi knock} down are very similar

Global DNA methylation decrease and growth delay (colony formation) are common to both Hiwi down-regulation and DNA-methyltransferase inhibitor treatment, but to address whether the specific mechanism is the same, we performed a temporal gene expression profiling (Affymetric U133 Plus 2.0 Arrays) on sh-Hiwi MFH cells after Hiwi down-regulation (via doxycycline induction) or 5-azacytidine treatment. Using unsupervised hierarchical clustering of the whole gene sets of both conditions, we find that early time points (24-48hrs) of Hiwi downregulation associate with early time points (24-48hrs) of 5-azacytidine treatment, and similarly, longer down-regulation (4-7d) of Hiwi associates with longer treatment with 5-azacytidine (Figure 4.6A). We imported the Broad GSEA Gene Sets (Cytogenetic, Functional, Regulatory, 
Neighborhood and Gene Ontology) into GeneSpring and comparatively analyzed the expression patterns of MFH cells at 24 hours treated with either 5-azacytidine treatment or dox-ind-sh-HiwiMFH at 1, 2, 4 or 7 days under similar treatments. Using a standard GSEA FDR (q value) of 0.25 we were unable to identify any differentially expressed gene sets.

Overlap of differentially expressed genes in both array sets shows that at early time points (Figure 4.6B), $75 \%$ of the genes that are differentially expressed following Hiwi down-regulation are also differentially expressed during 5-azacytidine treatment; and over $99 \%$ of these overlapping gene changes trend similarly. At later time points (Figure 4.6C), $50 \%$ of the genes that are differentially expressed following Hiwi down-regulation are also differentially expressed following 5-azacytidine treatment, with $93 \%$ of these overlapping gene changes trending similarly. These data suggest that 5-azacytidine treatment of MFH cells mimics Hiwi downregulation in terms of gene expression profiling. 


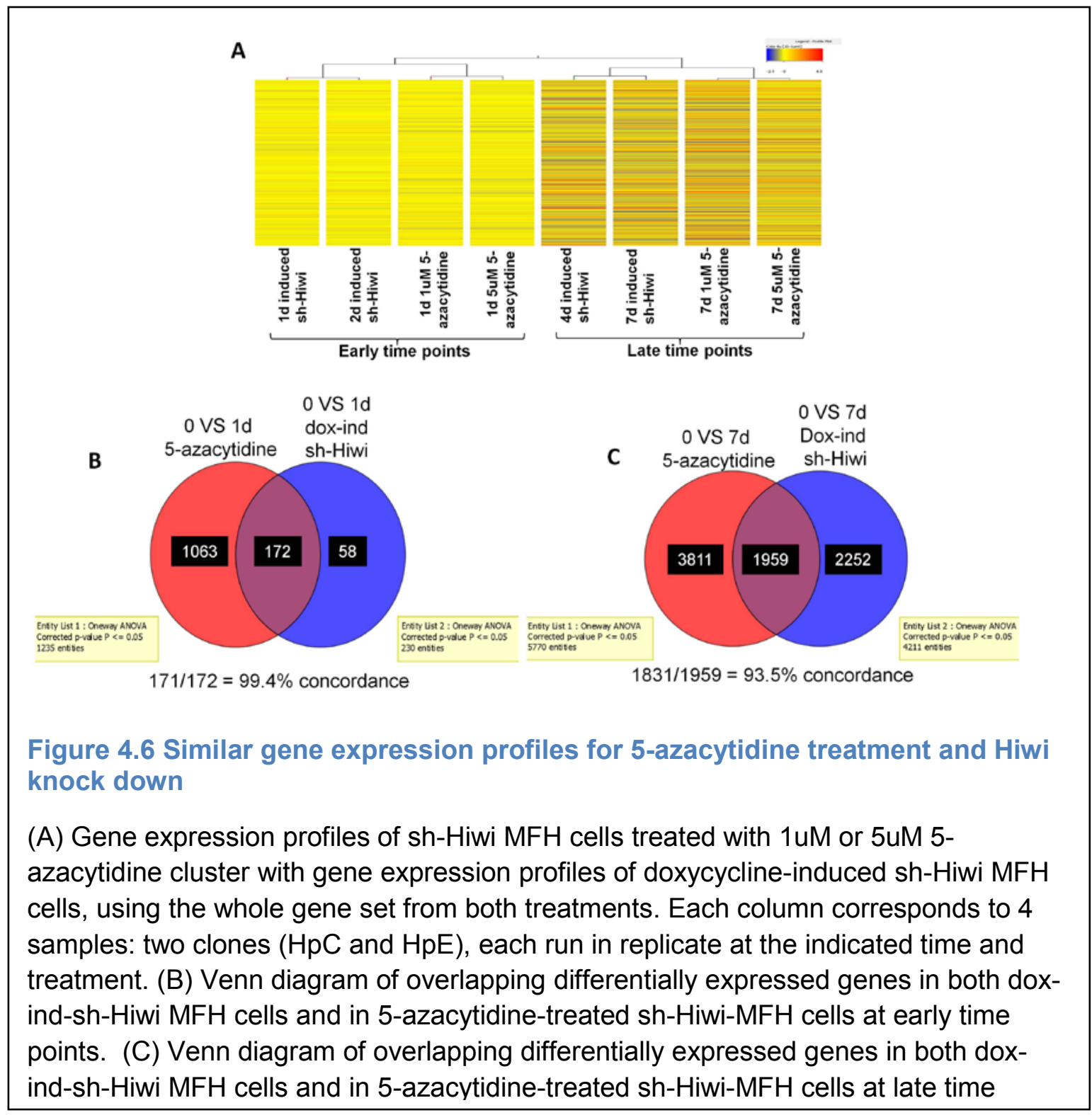

\section{Promoter methylation is unchanged by Hiwi expression}

The methylation of promoter region DNA is widely accepted as an epigenetic marker of transcriptional repression. The promoter region of a gene can vary, but it is generally considered to be the region upstream of the gene's transcriptional start site. An increase in the DNA methylation of the promoter region is an important mechanism for stable silencing of that gene. CpG islands are CpG-rich areas of 200bp to several kilo-bases in length, usually located in or near the promoters of highly expressed genes. Increased methylation of CpGs within the 
promoter region of tumor suppressor gene genomic DNA is a common epigenetic feature associated with tumorigenesis, and results in gene silencing $[114,115,116]$.

Upon observing a significant increase in global DNA methylation in Hiwi expressing MSCs (Figure 4.2A), we then asked which specific promoters might be affected by this increase in DNA methylation. In order to answer that question, we examined the methylation changes in 27,000 CpG islands covering 14,495 genes that occur during Hiwi down-regulation in doxycycline-inducible sh-Hiwi MFH cells using Illumina Meth27 arrays before and after 2, 4 and 7 days of doxycycline induction. Despite our data showing that global DNA methylation directly correlates with Hiwi expression (Figures 4.2 and 4.3), no overall change in CpG island methylation was observed following Hiwi down-regulation (Figure 4.7A). We did identify 18 CpG islands that decreased by at least $10 \%$ (i.e., beta>0.1) (Figure 4.7B) following Hiwi downregulation after 7 days in both of our doxycycline-inducible sh-Hiwi MFH clones C \& E (no significant changes in DNA methylation were observed at earlier time points).

We then asked if the methylation changes in the $\mathrm{CpG}$ sites of the 17 genes identified in Figure 4.7B correlate with gene expression changes observed in dox-ind-sh-Hiwi MFH cells. However, the 17 genes associated with these $18 \mathrm{CpG}$ islands did not show a simultaneous increase in gene expression following Hiwi down-regulation (Figure 4.8A) nor did they show a consistent decrease in Hiwi-MSCs as compared to parental MSCs (Figure 4.8B). The p-values of the differentially expressed genes show they are indeed statistically differentially expressed (Figure 4.9A, B). And finally an equal number of $\mathrm{CpG}$ islands can readily be observed that gain CpG methylation following Hiwi down-regulation (Figure 4.7A). These data suggest that CpG methylation status of genes (at least the 14,495 on the Illumina Meth27 array) are not affected; and thus that the overall change in global DNA methylation observed may be accounted by either DNA-methylation of repetitive elements (non-gene regions) or non-CpG gene methylation. 


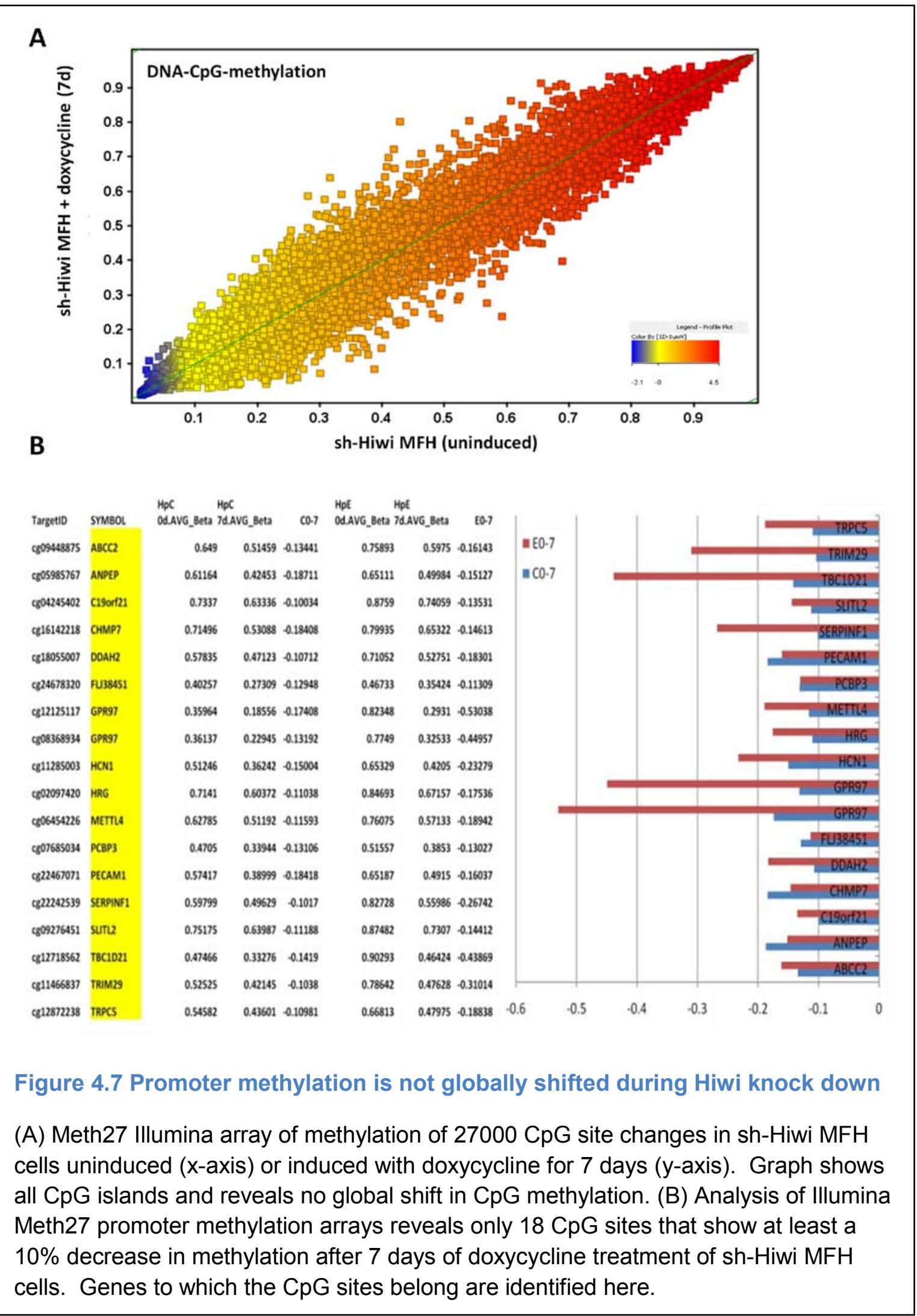


A. Probesetio

$\begin{array}{lrrrrrr}\text { Probesetio } & {[0]} & {[1]} & {[2]} & {[4]} & {[7]} & \text { Gene Svmbol } \\ \text { 206155_at } & -0.0733943 & -0.10680139 & 0.13196218 & 1.0416831 & 0.96938217 & \text { ABCC: }\end{array}$

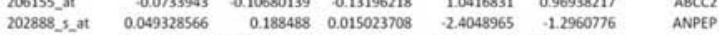

$\begin{array}{llllllll}234458 \text { zt } & -0.037112117 & -0.027178228 & -0.035865903 & 0.117394686 & -0.037916243 & \text { ANPEP }\end{array}$

$\begin{array}{lllllll}234576 \text { at } & 0.060286403 & 0.1316294 & -0.047082067 & 0.1250161 & 0.13646835 & \text { ANPEP }\end{array}$

$\begin{array}{lllllll}\text { 212925_at } & -0.06757116 & -0.110749125 & 0.08726919 & 0.16328168 & 5.826-04 & \text { C19ort21 }\end{array}$

$\begin{array}{llllllll}\text { 212313_at } & 0.12837815 & 0.13118327 & 0.15572846 & -0.10087514 & -0.3814268 & \text { CHMP7 }\end{array}$

$\begin{array}{lllllll}202262 \text { - X_at } & 0.36268783 & -0.030340672 & 0.1966418 & -0.849056 & -0.3269658 & \text { DOAH2 }\end{array}$

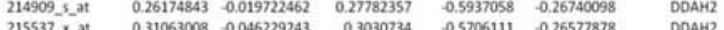

$\begin{array}{lllllll}215537 \times 210 & 0.31063008 & -0.046229243 & 0.3030734 & -0.5706111 & -0.26577878\end{array}$

$\begin{array}{llllllll}220404 \text { at } & 0.115902305 & 0.019161224 & 0.15976703 & 0.023174763 & 0.20661461\end{array}$

$\begin{array}{llllllll}1553723 \text { At } & -0.062339067 & 0.06559503 & 0.017951846 & 0.007705212 & -0.0191921\end{array}$

1556351 at $0.09817022 \quad 0.018560470 .047104597 \quad 0.097780764-0.052634895$

$\begin{array}{llllllll}1562563 \text { at } & 0.020771992 & 0.10293794 & 0.09129512 & 0.060242176 & 0.044439793\end{array}$

$\begin{array}{llllllll}206226 \text { at } & -0.18525189 & -0.047281027 & 0.07190704 & 0.12915313 & 0.09660435\end{array}$

$\begin{array}{lllllll}31835 \text { at } & -0.019147396 & -0.016732275 & -0.07945281 & 0.06539416 & 0.03663528\end{array}$

$\begin{array}{lllllll}219698 \text { __at } & 0.17287624 & 0.030663729 & 0.11150503 & 0.107884526 & 0.24561822\end{array}$

$\begin{array}{lllllll}232194 \_a t & 0.1388967 & 0.14859223 & 0.09223127 & .0 .3956975 & 0.1052711\end{array}$

$\begin{array}{lllllll}205663 \_a t & 0.2557118 & -0.6160629 & -0.19656909 & 0.32312965 & 0.2737435\end{array}$

$\begin{array}{lllllll}230486 \text { at } & 0.30437112 & -0.38833165 & -0.06989348 & 0.21682274 & -0.11991489\end{array}$

$\begin{array}{lllllll}233998 \text { __at } & 0.010836065 & -0.11168691 & 5.41 E-04 & 0.20387226 & 0.2608263\end{array}$

$\begin{array}{lllllll}208981 \text { at } & 0.1284442 & 0.14714658 & 0.39116204 & 0.57154286 & 0.9278921\end{array}$

$\begin{array}{lllllll}208982 \text { at } & 0.015222907 & -0.71100664 & -0.8831867 & 0.6052331 & 0.9734168\end{array}$

$\begin{array}{lllllll}208983 \text { sat } & 0.029506683 & -0.14076 & .0 .25303257 & 0.4846003 & 0.6695034\end{array}$

$\begin{array}{llllll}\text { 1559921_at } & 0.075879216 & 0.3595941 & -0.2813363 & 0.4266262 & 1.1869195\end{array}$

$\begin{array}{llllll}\text { 202283_at } & 0.14522845 & -0.1029315 & 0.05638349 & 0.09813738 & 0.119442105\end{array}$

$\begin{array}{lllllll}231475 \text { at } & -0.0657716 & 0.062285066 & 0.052489432 & 0.07202846 & 0.060444474\end{array}$

$\begin{array}{llllllllll}202504 \text { at } & 0.17232692 & 0.003016591 & 0.009980142 & 0.019295454 & 0.0574004\end{array}$

$\begin{array}{llllllll}211001 & -0.024261475 & -0.014928699 & 0.17543364 & 0.012040257 & 0.06951344\end{array}$

$\begin{array}{lllllll}2110023 \text { 3at } & -0.10054731 & -0.15538073 & 0.19021249 & 0.14809155 & 0.26196682\end{array}$

$\begin{array}{lllllll}220552 \text { at } & -0.03836572 & 0.03587365 & 0.043364525 & 0.013252437 & -0.095062196\end{array}$

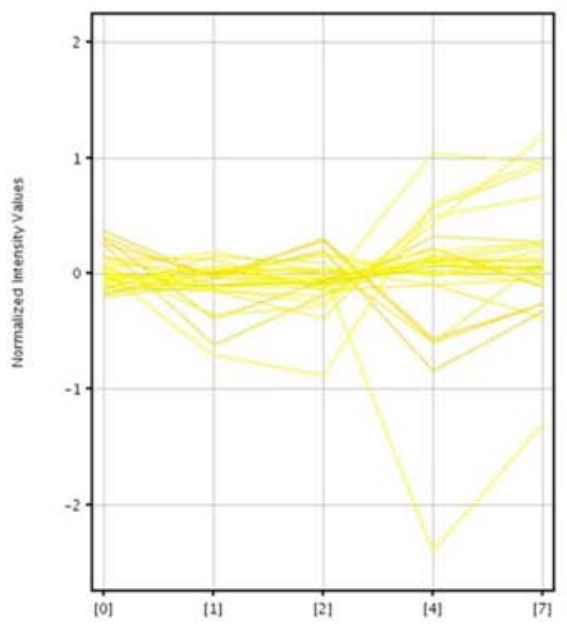

B.

$\begin{array}{rrr}\text { MSC } & \text { MSC-HIWI } & \text { Gene Symbol } \\ -0.1180708 & 1.0337815 & \text { Abcc2 } \\ 0.01937866 & 0 & \text { Anpep } \\ 0.53152585 & -0.5223172 & \text { Chmp7 } \\ -0.1644375 & 0.8971391 & \text { Chmp7 } \\ 0.52659893 & -0.5131321 & \text { Ddah2 } \\ -0.0494617 & 0.29964197 & \text { Gpr97 } \\ -0.0101488 & 0.29338968 & \text { Hcn1 } \\ -0.2320232 & 0.5606898 & \text { Hrg } \\ 0.51993585 & 0 & \text { Mettl } 4 \\ 0.5001662 & -0.7188717 & \text { Mettl } 4 \\ 0.17711353 & -0.39500022 & \text { Pcbp3 } \\ 0.27912688 & -0.03305185 & \text { Pcbp3 } \\ -0.1450533 & 0.032348394 & \text { Pecam1 } \\ 0.8888507 & -0.5064578 & \text { Serpinf1 } \\ 0.95619345 & -0.51589537 & \text { Serpinf1 } \\ 0 & 0.3359896 & \text { Tbc1d21 } \\ -0.3220557 & 0.78362906 & \text { Trpc5 }\end{array}$

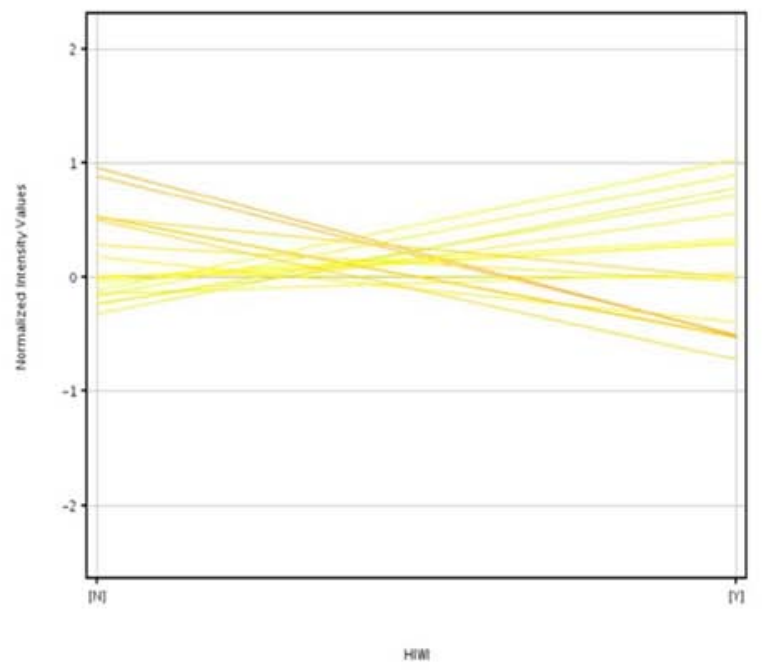

Figure 4.8 CpG methylated genes do not correlate to genes expression changes in sh-Hiwi MFH cells and Hiwi-MSCs

(A) Gene expression profiles of the 17 identified genes with CpG site hypomethylation, after $0,2,4$ or 7 days of doxycycline induction of sh-Hiwi MFH cells. While corresponding $\mathrm{CpG}$ sites are hypo-methylated, there is no corresponding increase in gene expression. (B) Gene expression profiles of the 17 identified genes with $\mathrm{CpG}$ site hypo-methylation in Hiwi-MSCs. Conversely, there is no decrease in expression of these genes. Because each of the 17 identified genes contains multiple spots on the array, corresponding to multiple Gene IDs, multiple rows for each gene are shown in both $(A)$ and $(B)$. 
A

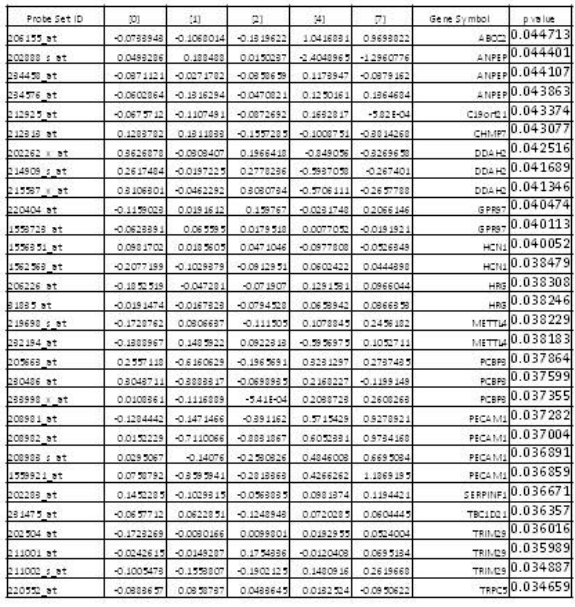

B

\begin{tabular}{|c|r|r|r|}
\hline MSC & MSC-HIW/ & Symbol & p-value \\
\hline-0.11807 & 1.033782 & Abcc2 & 0.039961 \\
\hline 0.019379 & 0 & Anpep & 0.039696 \\
\hline 0.531526 & -0.52232 & Chmp7 & 0.039477 \\
\hline-0.16444 & 0.897139 & Chmp7 & 0.039037 \\
\hline 0.526599 & -0.51313 & Ddah2 2 & 0.03877 \\
\hline-0.04946 & 0.299642 & Gpr97 & 0.038264 \\
\hline-0.01015 & 0.29339 & Hcn1 1 & 0.03752 \\
\hline-0.23202 & 0.56069 & Hrg & 0.037211 \\
\hline 0.519936 & 0 & Mettl4 & 0.036427 \\
\hline 0.500166 & -0.71887 & Mettl4 & 0.036102 \\
\hline 0.177114 & -0.395 & Pcbp3 & 0.036047 \\
\hline 0.279127 & -0.03305 & Pcbp3 & 0.034631 \\
\hline-0.14505 & 0.032348 & Pecam1 1 & 0.034477 \\
\hline 0.888851 & -0.50646 & Serpinf1 & 0.034422 \\
\hline 0.956193 & -0.5159 & Serpinf1 1 & 0.034406 \\
\hline 0 & 0.33599 & Tbc1d21 & 0.034365 \\
\hline-0.32206 & 0.783629 & Trpc5 & 0.034078 \\
\hline & & &
\end{tabular}

Figure 4.9 P-values of differentially expressed genes identified in CpG promoter methylation array

(A) p-values of differentially expressed genes identified in Figure 4.8A. (B) pvalues of differentially expressed genes identified in Figure 4.8B

8. Identification of methylation-dependent tumor suppressors via gene expression profiling

To explore further the potential relationship between Hiwi associated DNA methylation and the resultant effects on genes we performed global gene expression analysis and subsequently focused on a group of tumor suppressor genes (TSGs) [117] that were at least 1.5 fold: (1) down-regulated in Hiwi-MSCs as compared to parental MSCs; (2) up-regulated in dox-ind-shHiwi MFH cells following 7 days of doxycyline; and (3) to ensure that these genes were DNA methylation dependent, further up-regulated following 5-azacytidine treatment of MFH cells (Figure 4.10A). This overlap gave rise to 19 genes (Figure 4.10B). In each case of the three analyses in Figure 4.10A, the 1.5 fold differential fold expression was chosen as the discriminator. There was no statistical significance associated with fold change. Each of the three was then independently overlapped. Thus there is by definition no associated p-value. 
Since Rb1 was identified in this set and its methylation has been thoroughly studied, we further assayed its promoter methylation via bisulfite sequencing as well as the Line1 regulatory element (Figure 4.11). In agreement with our CpG promoter methyl array results, there are no changes in the methylation status of the Rb1 promoter $\mathrm{CpG}$ sites as Hiwi levels change (Figure 4.11, middle and bottom graphs). Additionally, we find that the methylation of Line1 transposon CpG sites also remain unchanged as Hiwi level change (Figure 4.11, top graph). These data suggest that because no methylation changes occur in these methylation-dependent genes, Hiwi-associated DNA methylation increase that we observe is likely promoter non-CpG site methylation. 
A
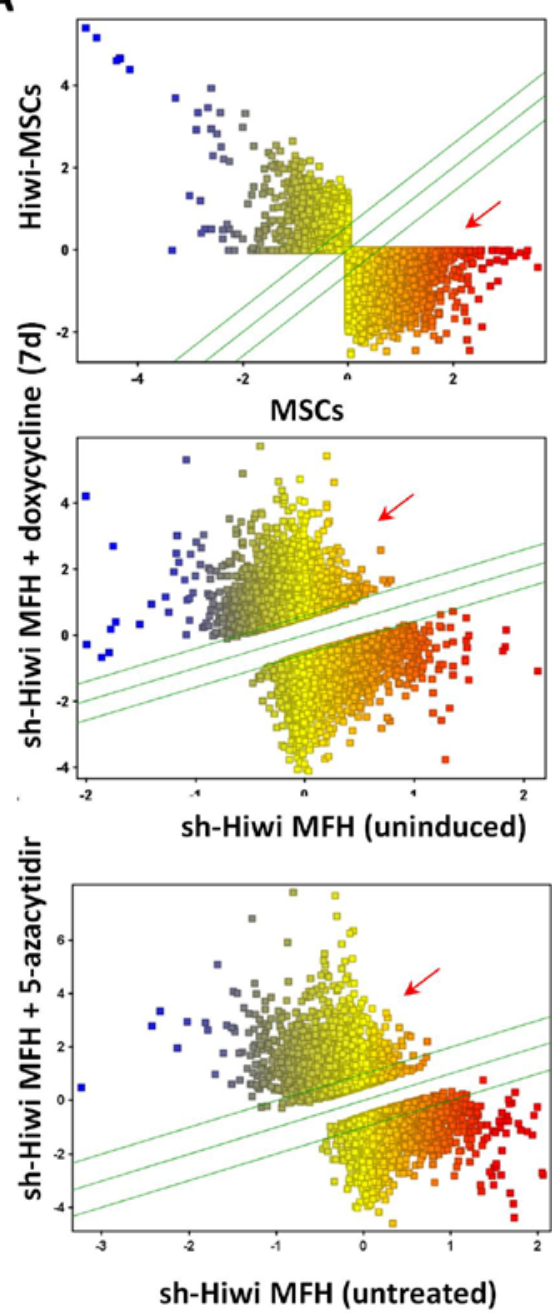

B

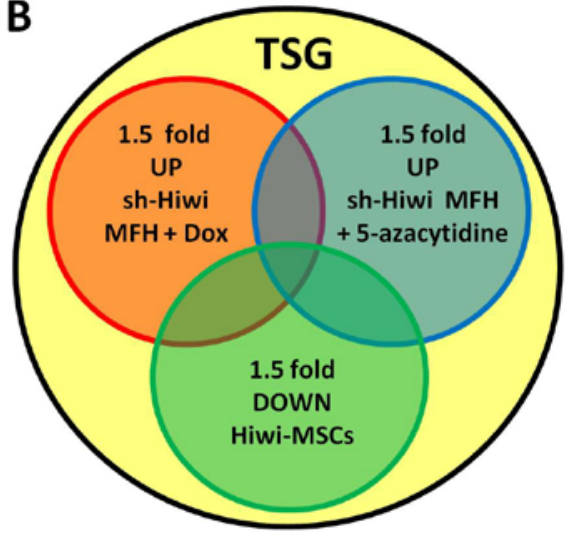

\begin{tabular}{|c|}
\hline Gene Symbol \\
\hline CDKN2B \\
\hline CDKN2C \\
\hline CLCA2 \\
\hline EFNA5 \\
\hline EPHB2 \\
\hline EZH2 \\
\hline ID4 \\
\hline IGFBP5 \\
\hline IGFBP7 \\
\hline LDOC1 \\
\hline LYPD1 \\
\hline MEF2C \\
\hline NFYA \\
\hline PHB \\
\hline PKN3 \\
\hline PLXNC1 \\
\hline Rb \\
\hline SMAD3 \\
\hline SOCS1 \\
\hline
\end{tabular}

Figure 4.10 Assessment of Hiwi target genes

(A) Top panel: Affymetrix 4302.0 array of gene expression changes in parental MSCs (x-axis) or Hiwi-MSCs (y-axis). Affymetrix U433 array of gene expression changes in sh-Hiwi MFH cells uninduced (x-axis) or induced for 7 days with doxycycline (y-axis) (middle panel) or untreated (x-axis) and after 7 days of $1 \mathrm{uM}$ 5-azacytidine (y-axis) (bottom panel). Arrow indicates genes used in overlap analysis. (B) Overlap of Tumor Suppressor Genes (TSG) as described. All 19 overlapping TSGs are listed here. Each comparison consists of two clones in replicates or four total samples. 


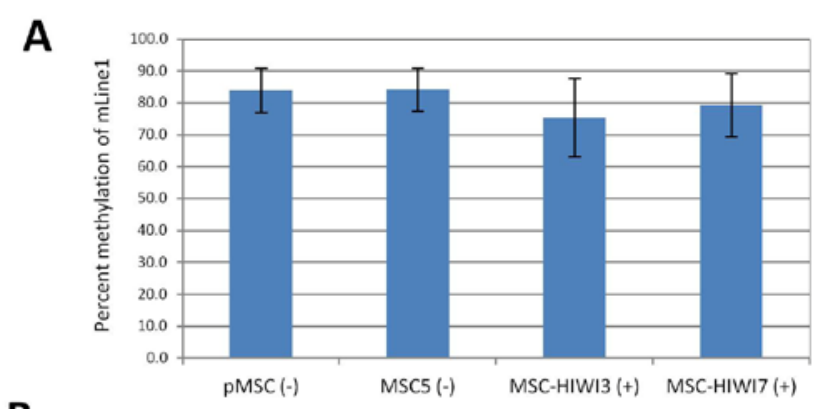

B

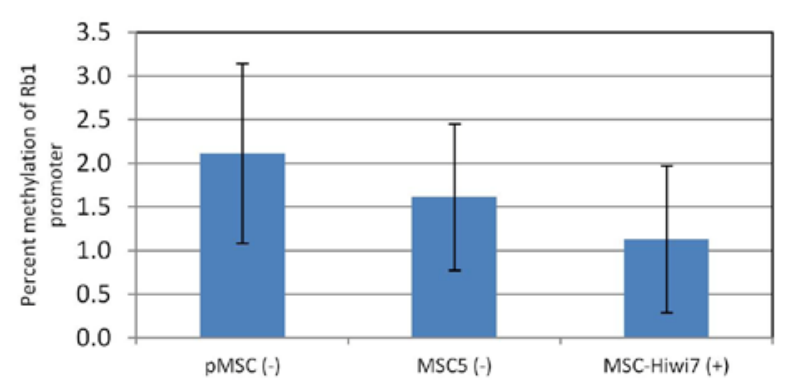

C

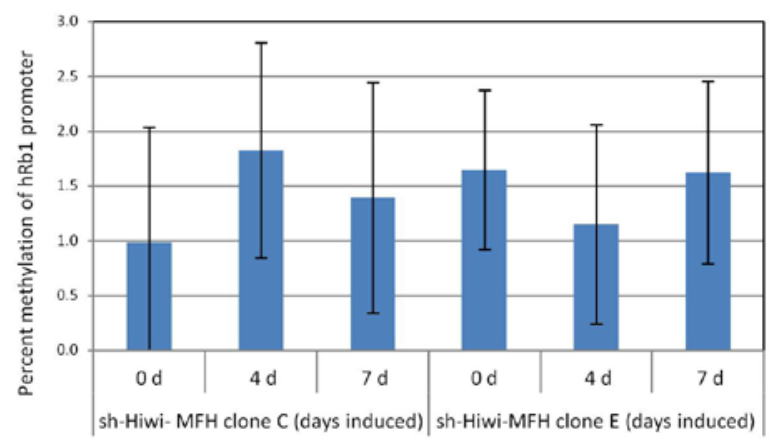

Figure 4.11 Methylation of Rb1 and Line1 promoters do not change as Hiwi levels change

Bisulfite sequencing of Rb1 promoter CpGs in Hiwi-MSCs (top graph) and in sh-Hiwi MFH cells (middle graph) reveal no methylation changes as Hiwi levels change.

Similarly, bisulfite sequencing of Line1 CpGs in Hiwi-MSCs (bottom graph) reveal no methylation changes as Hiwi levels change.

We went on to further examine the methylation of Line1 and IAP transposon regions before and after treatment with 5-azacytidine in Hiwi-MSCs (Figure 4.12). Although bisulfite sequencing of the Rb1 promoter and of Line1 did not show any changes in CpG methylation in Hiwi-MSCs, we performed additional quantitative PCRs followed by bisulfite conversion on HiwiMSCs that were treated with $5 \mathrm{uM}, 10 \mathrm{uM}$ or $50 \mathrm{uM} 5$-azacytidine for $18 \mathrm{~h}$. There are no significant 
changes in the methylation of these transposon regions in the Hiwi-MSCs. These quantitative PCR data of IAP and Line1 are in line with our bisulfite sequencing data for both Line1 and Rb1 (Figure 4.11), suggesting that global DNA methylation increases in Hiwi-MSCs, but not at sites that are local to Line1 and IAP (just as with $\mathrm{Rb} 1$ ). The methylation of these regions remains unchanged during 5-azacytidine treatment, further suggesting that Hiwi-associated methylation is non-CpG methylation. In agreement with our promoter CpG methyl array (Figure 4.7), no methylation changes at any CpG islands (increase or decrease) in these DNA methylation dependent genes were observed (Figure 4.8).

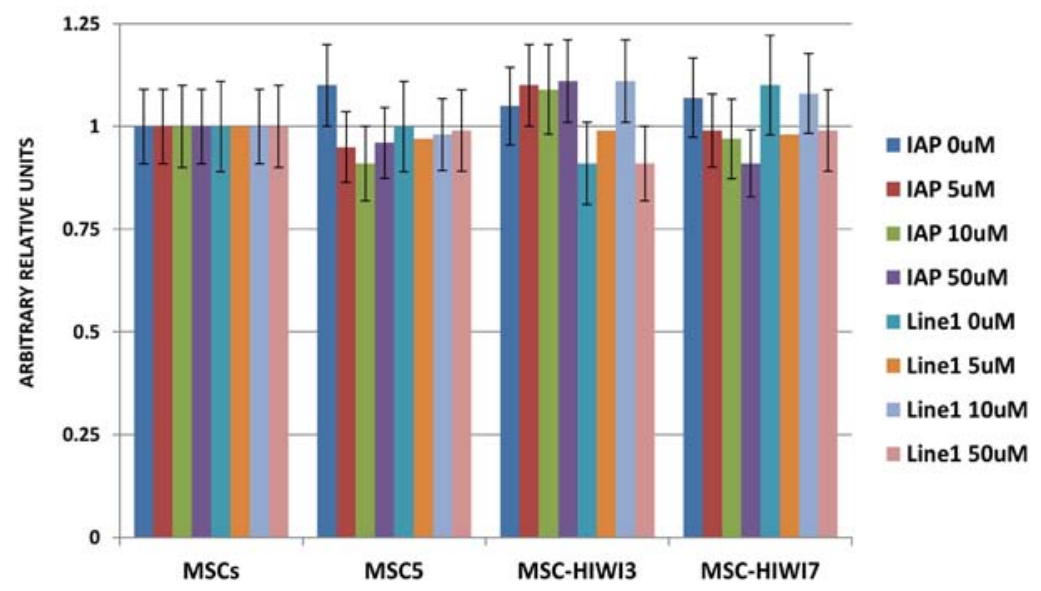

Figure 4.12 Line1 and IAP transposon methylation is unchanged in Hiwi-MSCs

Quantitative PCR of IAP or Line1 transposon expression, followed by bisulfite conversion, on parental MSCs, MSC5, Hiwi-MSC3 and Hiwi-MSC7 treated with the indicated concentration of 5-azacytidine for 18h. Experiments were performed in triplicate. Error bars represent standard error.

\section{Cyclin dependent kinase inhibitor expression is altered in Hiwi-expressing cells}

Since cell cycle genes were over-represented in the 19 TSGs selected, and upon further examination several additional cyclin dependent kinase inhibitors (CDKIs) could be identified immediately below our previously described threshold, we further focused on CDKIs p21, 
p27 and p15. Now that we had identified CDKIs as genes which are sensitive to Hiwiassociated DNA methylation by our in silico methods, we wanted to validate the above CDKIs as responsive at the protein level to Hiwi. CDKIs decrease in Hiwi-MSCs and increase in sh-Hiwi MFH upon doxycycline induction (Figures 4.13A, B).

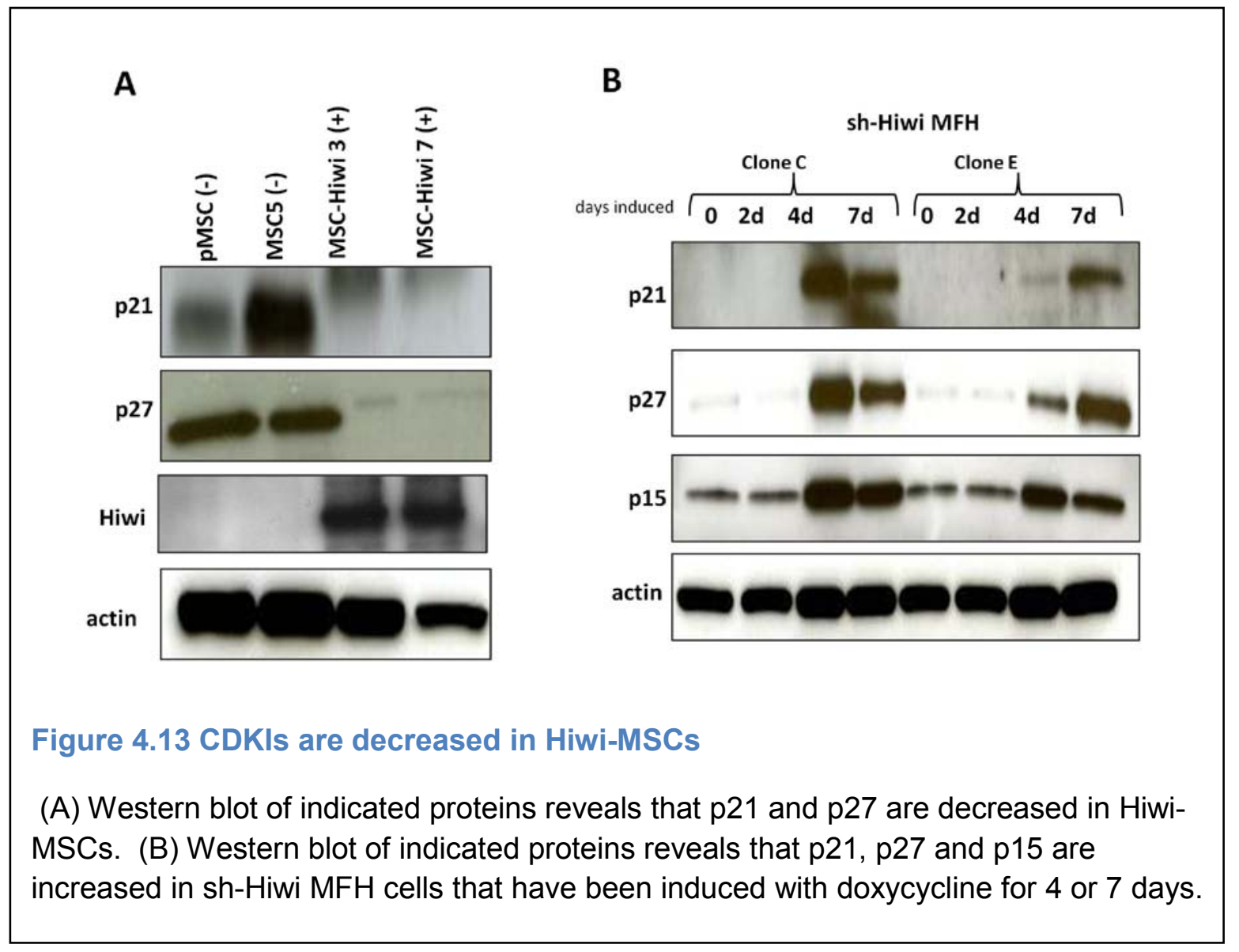

We further performed IHC on the human sarcoma tissue microarray used to assess Hiwi (Figure 2.1); and show that p15, p21, and p27 show a tight IHC-based inverse correlation to Hiwi levels (Figure 4.14). Ten cases of each subtype (present in triplicate) were scored from 0 to 3 blindly by sarcoma pathologists for each of the indicated proteins. Average scores are plotted here. Importantly, another CDKI, p16, which was not identified in our screen, does not show such a correlation at the IHC level. Previous analyses have only inversely linked p27 to 
sarcoma grade [118]. Thus our demonstration of an inverse relationship to Hiwi for p15, p21 and p27, combined with our functional data, leads us to conclude that our observations in model systems apply to human sarcomas.
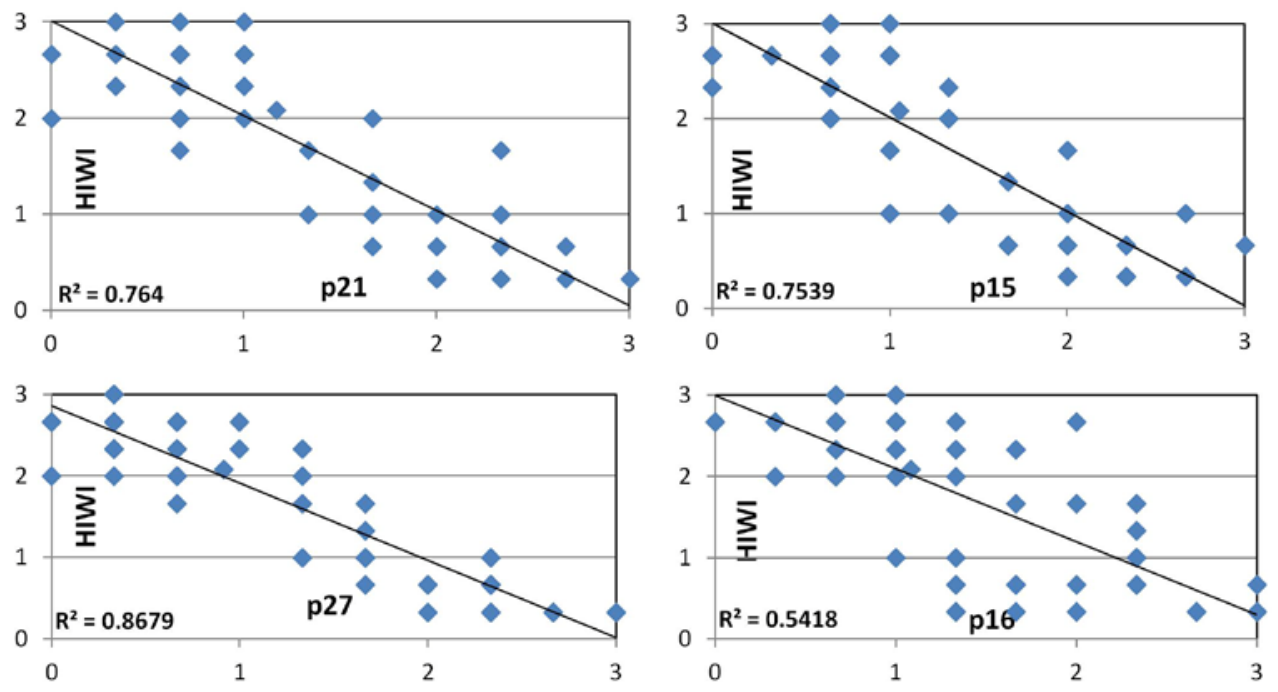

Figure 4.14 CDKls have an inverse relationship with Hiwi in primary human sarcomas

IHC analysis of a human sarcoma TMA reveals a tight inverse correlation of p21, p27 and p15 expression ( $x$-axes) to Hiwi expression ( $y$-axis) $\left(R^{2}=0.764 ; R^{2}=0.8679 ; R^{2}=0.7539\right.$, respectively) but no such correlation for $\mathrm{p} 16$ expression ( $\mathrm{x}$-axis) to Hiwi expression ( $\mathrm{y}$-axis) $\left(R^{2}=0.5418\right)$. Ten cases of each subtype (present in triplicate) were scored from 0 to 3 blindly by sarcoma pathologists. Average scores for each case are plotted here.

\section{Histone methylation marks remain unchanged during Hiwi knock down}

Our inability to detect any DNA promoter methylation via sequencing at either the Line1 element or at the Rb promoter (two transcripts heavily regulated by Hiwi) (Figure 4.11) leads us to suggest that promoters are not methylated. We then asked if other epigenetic modifications, besides promoter DNA methylation, might be altered in our sh-Hiwi-MFH cells. On this note a recent report by Sugimoto et al [119] found that another Hiwi family member, Hiwi2, induces p16 silencing via histone 3 lysine 9 methylation, but not, however, gene promoter DNA methylation. 
To examine the possibility of Hiwi-mediated histone methylation changes, we performed immunofluorescence staining for various histone marks (Figure 4.15A-F) on our dox-ind-shHiwi MFH cells. However, we were not able to discern any alterations in any histone marks examined during Hiwi down-regulation, although we do not doubt that just as reported by Sugimoto et al, promoters of silenced genes will be associated with silencing chromatin marks. We acknowledge that these are global analyses which examine histone marks at a low resolution. However, without discernible changes in a specific histone mark identified in this screening approach, we were unable to move forward with targeted analyses in a lead candidate. This is further explained in the discussion section of this chapter. 


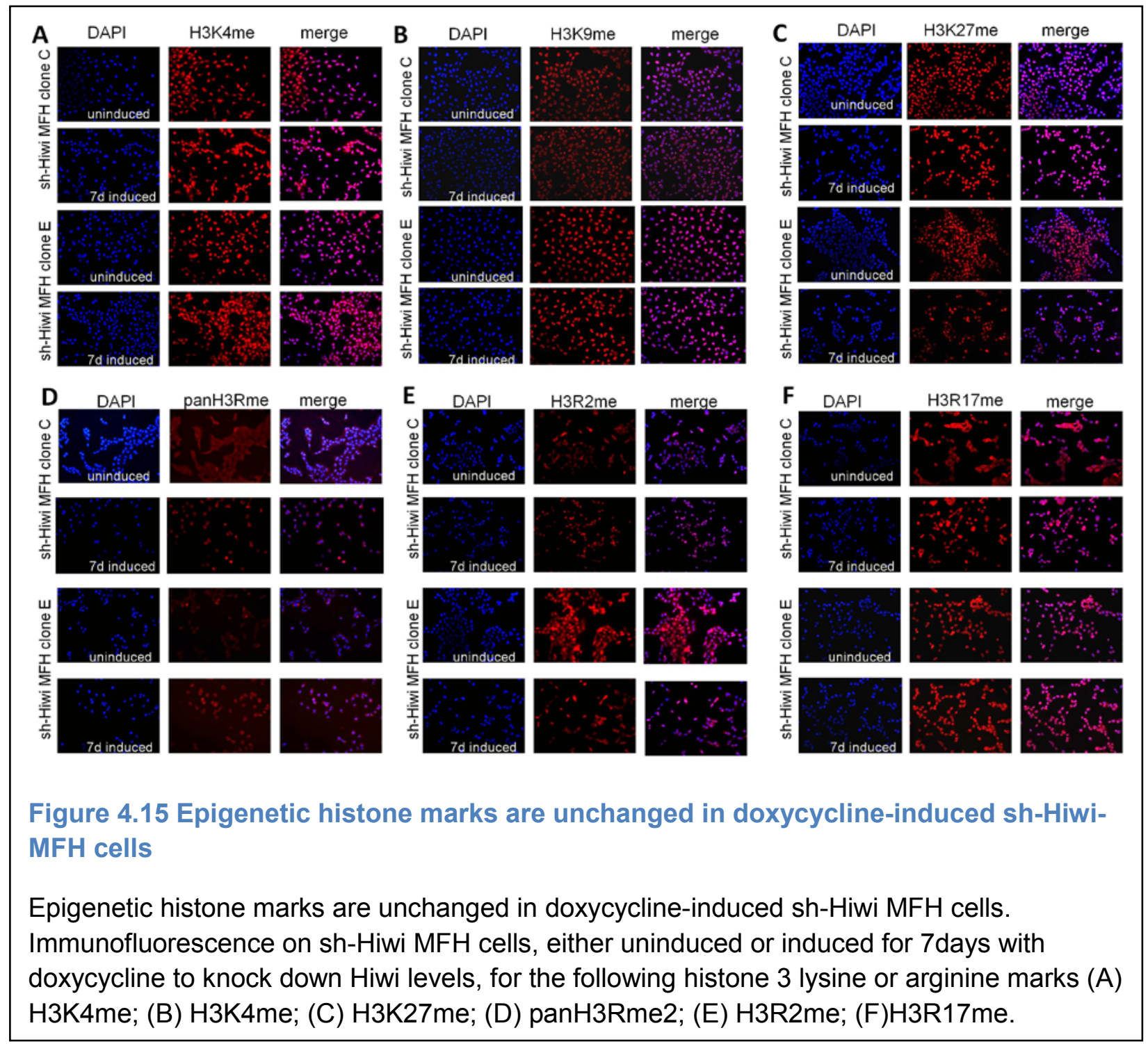

\section{Determining methylated loci via STAMP assay}

Taken together, our data suggest that global DNA methylation increases as Hiwi expression increases, but not at promoter regions. In order to identify which regions are methylated in Hiwiexpressing cells, we have undertaken a collaboration with the Scandura Lab (Weill Cornell Medical College). An assay developed in the Scandura lab, called a STAMP assay [120] (Sequence Tag Analysis of Methyation Patterns) is used to map methylated DNA regions to a 
reference genome in order to identify highly methylated genomic loci. We have employed this technique in our sh-Hiwi-MFH cells, either uninduced, or induced with doxycycline to downregulated Hiwi. Preliminary data suggest that there are about 200 differentially methylated regions in dox-induced sh-Hiwi-MFH cells, however, there is little correlation of these regions to gene expression changes. Because these data are highly preliminary and represent a noncomprehensive analysis of the genome, we are continuing to analyze these data rigorously. This work in on-going in both our lab and in the Scandura lab.

\section{Discussion}

Using primary mesenchymal stem cells, MFH sarcoma cells, and human tumor samples we show here that Hiwi mediated tumorigenesis is associated with global DNAhypermethylation and is reversible using DNA-methyltransferase inhibitors. We also show that Hiwi associated global DNA-hypermethylation occurs at non-promoter CpG regions. Additionally, Hiwi levels correlate inversely with levels of known tumor suppressor genes, specifically CDKIs. To the best of our knowledge, this is the first mechanistic examination of Hiwi functionality in a mammalian cancer context. Our studies reveal not only a novel oncogenic role for Hiwi as a driver of tumorigenesis (Chapter 2), but also that the use of epigenetic agents such as 5-azacytidine may be clinically beneficial for treatment of tumors that express Hiwi. Our data also show that Hiwi-mediated sarcomagenesis is associated with DNA hyper-methylation with subsequent genetic and epigenetic changes that favor a tumorigenic state, reconciling the outstanding conundrum of how Hiwi may act appropriately to promote genomic integrity during early development (via transposon silencing) and inappropriately during aberrant expression in adult tissues with subsequent tumorigenesis. Our data are the first to reveal a connection between Hiwi expression in human cancers and DNA methylation.

Despite the tight correlations between Hiwi expression, global DNA methylation, and tumorigenesis provided herein; several pieces still need to be elucidated. In order to identify the 
exact genomic loci which are methylated in our models, we are currently working in collaboration with the Scandura lab at Cornell on a genome-wide methylation analysis assay. Additionally, because our studies are the first to link Hiwi expression in cancer with DNA methylation, it will now be interesting to evaluate the DNA methylation status of all Hiwi expressing cancers, including human primary undifferentiated sarcoma samples. In our models and others $[73,121]$ Hiwi is predominantly cytoplasmic. Furthermore, the protein-level upregulation of DNMT1, DNMT3a and MDB2 (Figure 4.3) is post-transcriptional (RNA levels do not change in either Hiwi-MSCs compared to parental cells or in sh-Hiwi MFH before and after doxycycline induction, data not shown). These data together, and given previous reports of Hiwi's interaction with ribosomes [122] and its cytoplasmic localization, Hiwi-associated DNAmethylation may involve as yet unidentified control of DNMT translation. The exact relationship between Hiwi and its mechanistic epigenetic control is an ongoing endeavor both in our laboratory within a cancer context and in the laboratories of many others interested in the role of Piwi proteins in development $[2,28,29,30,36,55,58,59,60]$.

Even in mice, where Piwi orthologs have been more extensively studied in terms of DNA methylation $[55,59]$, the exact mechanism by which Piwi family members mediate DNA methylation during normal embryological development is still unclear. Our data that (1) Hiwi translationally up-regulates DNMTs, (2) global DNA methylation (at CpG and non-CpG sites) correlate directly with Hiwi levels, (3) promoter CpG methylation does not change during Hiwi down-regulation and (4) Hiwi down-regulation and 5-azacytidine treatment are mechanistically similar, taken all together, suggest that Hiwi-associated DNA-methylation is occurring globally at non-CpG promoter areas and/or at repetitive element regions. Our inability to detect any DNA promoter methylation via sequencing at either the Line1 element or at the Rb promoter (two transcripts heavily regulated by Hiwi) leads us to suggest that promoters are not methylated. DNA methylation at repetitive element regions has been previously reported to result in long 
distance gene silencing via chromatin remodeling $[123,124]$ and given previous reports that Piwi proteins associate with piRNAs that share homology to repetitive elements $[61,125]$ may explain the lack of gene promoter methylation observed in our systems. A recent report [119] suggests that Hiwi2, another Piwi protein, induces p16 silencing via histone 3 lysine 9 methylation, but not, however, gene promoter DNA methylation. We examined possibility of Hiwi-mediated histone methylation changes during Hiwi knock down in MFH cells (Figure 4.15A-F), but histone methylation remains unchanged. We are aware that immunofluorescence (IF) is perhaps a low resolution method to detect histone methylation changes. However, using this IF methodology to screen through many histone marks, we were unable to identify a lead to carry further to more time-intensive high-resolution types of analysis, such as western blotting for post-translational histone marks [126] or Chromatin Immunoprecipitation-sequencing (ChIP-seq) [127] to identify histone marks and which genomic elements they associate with. Without a substantial lead histone modification to investigate further, we interpret our data to suggest that Hiwi does not mediate histone methylation marks during doxycycline-induction of Hiwi knockdown in MFH sarcoma cells. Regardless, we believe that stringent statistical cut-offs and the overlapping of gene lists from several independent analyses, clones, and model organisms, resulted in a gene list that likely belies the true extent of gene silencing found in Hiwi expressing cancers. The identified genes are likely to be extremely specific and serve as the basis for further studies of Hiwi mediated gene regulation, tumorigenesis and DNA methylation.

Our observation that Hiwi-mediated tumorigenesis is associated with increased global DNA methylation is somewhat discordant from the more widely accepted notion that global methylation levels are decreased in a variety of human cancers despite specific increase in both promoter and non-promoter CpG island methylation. Thus the global hypomethylation observed in cancer is believed to predominantly occur via repetitive element hypomethylation (which accounts for a significant portion of the human genome; reviewed extensively in $[128,129])$. As a 
result of its inherent developmental preference for repetitive element silencing via DNA methylation, Hiwi may preferentially target repetitive elements for methylation in cancer cells thus mechanistically accounting for an exception to the commonly observed global hypomethylation of cancer. Ours are thte first data to examine DNA methylation levels in Hiwiexpressing cancers.

Although many recent reports detect high levels of Hiwi in all cancers examined, none have addressed a role for Hiwi in cancers, nor do they investigate the mechanism by which Hiwi may be acting. The data presented here are, to our knowledge, the first to elucidate that DNA methylation dependent silencing of tumor suppressor genes accounts for the Hiwi-mediated tumorigenesis. Moreover, we have identified a potential therapeutic avenue in which Hiwi expressing cancer cells treated with 5-azacytidine show reduced colony forming capacity and share many gene expression changes with Hiwi knock down cells. This suggests a therapeutic rationale for treating patients with Hiwi-expressing tumors (which are sarcomas, but many other cancers as well) epigenetically by means of DNA-methyltransferase inhibitors. 


\section{Materials and Methods}

\section{Gene expression profiling}

RNA from the indicated cell lines were hybridized to Affymetrix HG U133 (human) or Affymetrix 4302.0 (mouse) oligonucleotide arrays per standard protocols of the Columbia Genomics Core Facility. Class-comparison analysis using two-sided Student t-tests identified mRNAs that were differentially expressed between indicated samples $(p<0.05)$. Raw data will be deposited in the public repository.

\section{Promoter methylation profiling}

Promoter methylation profiling was done using Illumina Meth27 promoter arrays. Samples were run at Roswell Park Cancer Institute Genomics Facility. Average beta values, corresponding to amount of methylation, were then analyzed using Genespring software.

\section{Colony Formation Assays}

Cells were suspended in $0.3 \%$ agar (Sigma) in culture medium and plated into 6 well plates, with a base layer of $0.6 \%$ agar in culture medium. For doxycyline and 5 -azacytidine treatments, cells were pre-treated for 7 days in culture before beginning colony formation assay. Cells were kept in drug-spiked media during the assay and monitored for colony formation. Pictures were taken after 4 weeks. Experiments were performed in 2 times, each time in duplicate.

\section{Semi-quantitative RT PCR}

Total RNA was extracted from the indicated cell lines using RNeasy RNA extraction kit (Qiagen) according to the manufacturer's protocol. $1 \mu \mathrm{g}$ of RNA was transcribed into cDNA using SuperScript III First Strand Synthesis System for RT-PCR (Invitrogen). To assess the expression levels of IAP, Line1, and $\beta$-actin control semi-quantitative RT-PCR reactions containing 
Platinum Blue PCR mix (Invitrogen), 1ul cDNA and corresponding primers were run at the following PCR program: $95^{\circ} \mathrm{C}$ x $2 \mathrm{~min} ; 95^{\circ} \mathrm{C} \times 30 \mathrm{~s}, 55^{\circ} \mathrm{C} \times 30 \mathrm{~s}, 68^{\circ} \mathrm{C}$ x $45 \mathrm{~s}$ for 28 cycles; $68 \times 5 \mathrm{~min}$. Experiments were performed in triplicate.

\section{Assessment of DNA methylation levels}

Genomic DNA was isolated by DNeasy Blood and Tissue Kit (Qiagen). Global DNA methylation levels were assessed by Methylflash Methylated DNA Quantification Kit (Epigentek) and read on a plate reader at $490 \mathrm{~nm}$, according to manufacturer's protocol. Experiments were performed in triplicate.

\section{Bioinformatic Analysis}

Total RNA was extracted from the indicated cell lines using an RNA extraction kit (Qiagen) according to the manufacturer's instructions. RNA was hybridized to Affymetrix U133Plus2 arrays via Genomics Shared Resource at Columbia University and analyzed as previously described by us $[9,10]$ and being submitted to NCBI GEO. Specifically, data was imported into GeneSpring and RMA (Robust Multi-array Analysis) was used to normalize and summarize probe-level intensity measurements (PM) corrected for background using a non-linear correction, done on a per-chip basis based on the distribution of PM values. Expression values were then quantile normalized. For RMA, the probe affinity effects are assumed to sum to zero, and the gene effect (expression level) is estimated using median polishing thus protecting against outlier probes. Hierarchical cluster analysis was performed using a Pearson correlation coefficient. Gene ranking per subtype was performed using ANOVA (parametric test, Benjamin and Hochberg false discovery rate $\mathrm{p}<0.05$, and Student-Newman-Keuls post hoc analysis). Statistical analysis to correlate stages of differentiation using analysis of variance and principle of component analysis was performed using GeneSpring Software as previously described in 
detail by us[9,10]. Broad GSEA Gene Sets (Cytogenetic, Functional, Regulatory, Neighborhood,a nd Gene Ontology) into GeneSpring and comparatively analyzed using a standard GSEA FDR (q value) of 0.25 .

\section{Immunohistochemistry (IHC)}

IHC was performed as previously described by us[93]. Briefly, formalin-fixed, paraffin-embedded tissues were rehydrated and treated with citric buffer for antigen retrieval. Slides were blocked with $10 \%$ horse of goat serum in $2 \%$ BSA-PBS and then incubated in primary antibody (diluted in $2 \%$ BSA-PBS) overnight at $4^{\circ} \mathrm{C}$. Following 30 minutes of secondary antibody and tertiary antibody (Vector Labs) incubation, slides were developed with 3,3-Diaminobenzidine (DAB) and counterstained with Hematoxyalin. For cells, fixation was performed with $50 \%$

Methanol/Acetone for 10 minutes, then blocking, primary antibody incubation, and detection was performed as for FFPE IHC. Primary antibodies used in these studies: Hiwi (Abcam, 12337); p15 INK4b (Novus Biologicals, NB100-91906); p16 (BD Pharmingen, G175-405); p21 (Santa Cruz, sc-6246); p27 (Santa Cruz, sc-528).

\section{Immunofluorescence (IF)}

Standard IF technique was used. Briefly, cells were fixed in cold 1:1 Methanol-Acetone and blocked with $10 \%$ donkey serum in $2 \%$ BSA-PBS. The following primary antibodies were applied overnight at $4^{\circ} \mathrm{C}$ at 1 to 200 dilution in blocking buffer: H3K4me (abcam \#ab8898); H3K4me (abcam \#ab6000 ); H3K27me (abcam \#ab6002); panH3Rme2 (abcam \#ab413); H3R2me (abcam \#ab80075); H3R17me (abcam \#ab8284).

\section{Sarcoma Tissue Microarray (TMA)}


The TMA contained 10 cases of each sarcoma subtype in triplicate. For TMA analysis, IHC staining was scored on a scale of 0 to 2 by multiple sarcoma pathologists. The average score for each sarcoma subtype was calculated and representative pictures are shown. Scoring of the TMA for CDKI expression as compared to Hiwi expression used a scale of 0 to 3.

\section{Western Blotting}

Standard western blotting technique was used. Briefly, protein lysate was collected from cells with RIPA buffer (Boston BioProducts) and 50ug were run on a 4-20\% Tris-glycine gradient gel (Invitrogen). Nitrocellulose membranes were incubated overnight at $4^{\circ} \mathrm{C}$ in primary antibody: DNMT1 (1:500, abcam 92453); DNMT3a (1:500, abgent AP1034a); MDB2 (1:1000, abcam 38646); Hiwi (1:500 ProSci 45-735P).

\section{Bone and Fat Differention}

Bone or fat differentiation was assessed in MSCs after 21 days in bone or fat differentiation media, as previously published [9]. Approximate percentage of differentiated cells was calculated based on the average alizarin red s or oil red o staining over 3 independent experiments. 


\section{Chapter 5: Conclusions and Future Directions}

In this set of studies, we use mesenchymal stem cells, sarcoma cells, a transgenic mouse model and primary human sarcoma samples to show that: (1) Hiwi is directly tumorigenic; (2) Hiwi-expressing tumors may be addicted to Hiwi expression; (3) Hiwi mediated tumorigenesis is associated with global DNA-hypermethylation and is reversible using DNA-methyltransferase inhibitors; (4) Hiwi associated global DNA-hypermethylation occurs at non-promoter CpG regions; and (5) Hiwi levels correlate inversely with levels of known tumor suppressor genes. This is the first mechanistic examination of Hiwi functionality in a mammalian cancer context. Our studies reveal not only a novel oncogenic role for Hiwi as a driver of tumorigenesis, but also that the use of epigenetic agents may be clinically beneficial for the treatment of tumors that express Hiwi. Additionally, our data show that Hiwi-mediated methylation is associated with DNA hyper-methylation with subsequent genetic and epigenetic changes that favor a tumorigenic state. This explains how Hiwi may act appropriately to promote genomic integrity during early development (via transposon silencing) but inappropriately during its aberrant expression in adult tissues with subsequent tumorigenesis. We have also identified the presence of piRNAs in parental MSCs and in Hiwi-expressing MSCs, though the presence of Hiwi does not cause a characteristic shift in the piRNA population. We went on to analyze corresponding locations in the genome of these piRNAs and found that they generally do not correlate to gene regions.

\section{A novel oncogenic role for Hiwi}

In Chapter 2, we address a possible role for Hiwi in stem cell maintenance and/or sarcomagenesis. We find that Hiwi is highly expressed in human primary sarcomas and that exogenous over-expression of Hiwi in sarcoma precursors (ie, mesenchymal stem cells) is able to block mesenchymal differentiation and promotes tumorigenesis in our xenograft model. 
These data, along with our Hiwi knock-down model in MFH sarcoma cells, are the first to reveal a novel oncogenic role for Hiwi.

Piwis have been studied in the fly (Piwi) and mouse (Miwi) germline. During normal development, they are thought to be critical for maintenance of germline stem cells. Overexpression of Piwi in flies results in increased germline stem cell numbers, and loss of Piwi results in decreased germline stem cell numbers [32,33]. Similarly, mice which do not express Miwi or Mili show a block in spermatogenesis [44,73]. In exploring the stem cell maintenance role for Hiwi outside the germline, and in the context of adult tissue stem cells, we explore the capacity of mesenchymal stem cells which exogenously over-express Hiwi to differentiate down the adipocytic or osteogenic pathways, two typical mesenchymal lineages. Hiwi-MSCs show a block in differentiation down both of these pathways, suggesting that Hiwi, when aberrantly expressed outside the germline - in this case in sarcoma precursor cells_-inhibits differentiation and promotes sarcomagenesis. In line with those data, conditional knock down of Hiwi in MFH sarcoma cells, derived from an undifferentiated sarcoma, results in re-establishment of osteogenic differentiation of the MFH cells. Further supporting the suggestion that Hiwi inhibits differentiation and promotes sarcomagenesis is our data which finds Hiwi is most highly expressed in high grade undifferentiated human sarcomas (HGUS) but is expressed at much lower levels in well differentiated sarcoma subtypes.

We have found also that Hiwi drives tumorigenesis, revealing a novel oncogenic role for Hiwi. When we began these studies [130], Hiwi was known to be highly expressed in sarcomas at the RNA level [26] and in gastric cancer [69]. Since the start of our investigations, many more reports have found high levels of Hiwi in a variety of cancers $[65,66,67,96]$. Importantly, all of these studies have focused on the clinical significance of Hiwi as a prognostic marker and none have addressed an oncogenic role for Hiwi in these cancers. In our xenograft model, Hiwi 
expression promotes sarcomagenesis. Similarly, conditional knock down of Hiwi reduces colony forming capacity, a common proxy for tumorigenic potential. Because ours are the first data to examine Hiwi as a driver of tumorigenesis, rather than simply a prognostic marker, we also asked if there is a direct genetic basis for Hiwi up-regulation in cancer. We found that there is no characteristic chromosomal amplification of Hiwi in Hiwi-expressing human sarcoma samples. Hiwi cDNA has been independently isolated from several human cancers and developing sperm $[64,69]$ without sequence divergence; thus although we cannot rule out a stabilizing mutation, it seems unlikely. Since part of the tumorigenic process involves a recapitulation of the embryonic state, similar mechanisms that up-regulate Hiwi during embryological development may result in Hiwi's up-regulation in cancer. Regardless, our data suggest that Hiwi inhibits differentiation and promotes sarcomagenesis when expressed outside the germline, in a cancer context.

Since our data are the first to suggest an oncogenic role for Hiwi, it will be critical to confirm that Hiwi acts similarly in the other cancers where it is highly expressed. Another open question is what the genetic basis for Hiwi over-expression is in primary sarcomas, as well as other high-Hiwi cancers. We have ruled out a chromosomal amplification of Hiwi in sarcomas, but an exhaustive sequencing effort of Hiwi in all cancers which express Hiwi might then be able to definitively discern any activating or stabilizing mutations. Identifying the upstream pathway components which up-regulate Hiwi in cancers will also be critical to more specifically defining Hiwi's oncogenic role, although analogous mechanisms which control Hiwi expression in germ stem cells during development are unknown. One approach to parsing out upstream proteins which control Hiwi expression is to identify which transcription factors (other members of a protein complex) may be associated with the Hiwi gene, perhaps through an immobilized template recruitment assay approach [131]. 


\section{Presence of piRNAs in mesenchymal stem cells}

A large focus of piRNA research has been dedicated to elucidating their role in germline stem cells. piRNAs were isolated from rodent testes by 4 different groups simultaneously $[54,56,57,70]$, suggesting that this novel class of small RNAs were specific to male germline stem cells. Additionally, the zebrafish Piwi homolog, Ziwi, is expressed exclusively in the gonad [71], further suggesting that the piRNA-Piwi pathway is specific to the germ-line. However, piRNAs have been identified outside germ stem cells, including in somatic cells of the Drosophila ovary [78], in neurons of the mouse central nervous system [79], in HeLa cells [81], in adult human brain and testes samples [101] and even in mesenchymal stem cells[83]. The piRNAs associate with Mili in the cytoplasm of mesenchymal stem cells and are thought to be important for keeping cellular proliferation in check. But, importantly, Wu and colleagues [83] examined Mili expression by immunoprecipitation (IP) and western blot using the same Mili antibody, suggesting that actual Mili expression is much lower than the enriched band shown. There was no evidence that other murine Piwi orthologs are present in mesenchymal stem cells. This likely gives the impression that Mili expression is much higher than would be otherwise identified. It is possible that Mili is not even detectable without IP-western blot enrichment using the same Mili antibody. Moreover, there was no western blot of Mili expression without IP enrichment, nor was there any evidence of Miwi and Miwi2 expression (the other murine Piwi homologs). This is in line with our work which has shown that Mili, Miwi and Miwi2 are not readily detectable by RT-PCR from murine MSCs.

Using a small RNA sequencing approach, we asked whether piRNAs are present in our Hiwi-MSCs or in our parental MSCs. Because piRNAs are known to associate with Piwi proteins, we expected to identify piRNAs in our Hiwi-expressing MSCs. But to our surprise, a population of piRNAs was also identified in our Hiwi non-expressing MSCs. While this is not the first identification of piRNAs in mesenchymal stem cells, it is the first identification of piRNAs in 
mesenchymal stem cells that do not express any Piwi homologs. This is in keeping with the previous identification of piRNAs in MSCs [83], since the Mili identified in those MSCs is likely only an experimentally-enriched band rather than true Mili expression. The other murine Piwi homologs were not detected at all. Since our data suggest that piRNA expression can be achieved without Piwis in MSCs, this raises the question of how these piRNAs are generated in the absence of Piwi homolog expression. It is possible that there is another still-unidentified Piwi homolog in MSCs that is responsible for a ping-pong cycle-like amplification loop to generate piRNAs. Alternatively, there may be a non-amplification loop mechanism for piRNA biogenesis that has not yet been elucidated. One way to approach the latter possibility is to use a mass spectrometry approach to pull out a biotinylated piRNA of interest and its associated proteins and then isolate the associated proteins for mass spectrometric analysis for identification. However, this requires that there is already a specific piRNA of interest to focus on. It may also be possible to use a pool of biotinylated piRNAs of interest rather than only one. Another related experiment is to exogenously transfect primary piRNA transcripts (available from Exiqon) into cells and then isolate the mature piRNAs with any associated proteins.

Our piRNA data suggest to us that perhaps piRNAs are more widely expressed than previously thought. piRNAs were also identified in our Hiwi-MSCs. When the piRNA population in one Hiwi expressor is compared to the piRNA population in a Hiwi non-expressor, we find that there is no global shift in piRNA expression pattern. This is in contrast to the kind of global and characteristic piRNA shift seen in Mili or Miwi null murine testis [45]. Moreover, we found that the vast majority of piRNAs do not correlate to gene regions, suggesting that they do not regulate gene expression. This is in line with a large body of work which implicates piRNAs and Piwis in silencing of transposon repeat regions, but not genes $[45,59,132]$. In order to more exhaustively map the genomic locations of the piRNAs identified in our studies, we would like to correlate these data with genomic elements, such as methylated regions, chromosomal ends, 
transposons, etc. Our initial bioinformatic analyses (Chapter 3) have confirmed that piRNAs in MSCs do not map to gene regions, and do not show any particular chromosome bias. However, a more extensive bioinformatic analysis to further correlate these piRNAs to other genomic elements will be critical to understanding the role of these piRNAs globally in MSCs. Interestingly, there has only been one report [101] of a specific piRNA, piR_015520, whose exogenous expression of this piRNA is able to downregulate a protein-coding gene: melatonin receptor $1 \mathrm{~A}$ gene. This is the first, and only, report of piRNA-mediated regulation of a proteincoding gene, rather than a transposon repeat element and it has yet to be confirmed in additional studies. Taken together, our data suggest that although there is a piRNA population present in parental MSCs in the absence of Miwi (or Miwi2 or Mili) expression, it does not regulate gene expression and piRNA expression patterns do not shift in a global way in response to Hiwi. Moreover, the vast majority of piRNAs identified do not correlate to gene regions.

piRNAs have not been thoroughly studied in cancers, however a few preliminary studies suggest that individual piRNAs may be altered in cancers. A specific piRNA, piRNA 651, is aberrantly over-expressed in multiple cancers (gastric, colon, lung and breast cancer tissues), when compared with normal tissues [88]. Furthermore, inhibition of this piRNA in gastric cancer cell lines (another cancer in which Hiwi is over-expressed [69]) resulted in decreased cellular growth. The growth inhibition effect of the piRNA antagonist was modest, but these data suggest that there may be an important potential role for aberrant piRNA expression in contributing to over-proliferation in cancer cells. Individual piRNAs have also been detected in peripheral blood of patients with gastric cancer [89]. Two specific piRNAs, one of which was piRNA 651, were chosen for evaluation and both were significantly lower in peripheral blood from gastric cancer patients, compared to healthy controls, suggesting that piRNA detection might be a valuable biomarker for identifying circulating gastric cancer cells. This report seems 
to contrast with the report that piRNA 651 is increased in cancer tissues, however this may be reflective of the different tissues which were examined for piRNA 651 expression: cancer tissues versus peripheral blood. Because these are only the first studies to examine piRNA expression levels in cancer, it is important to note that much more investigation is necessary, both to validate these studies and to identify piRNA expression in additional cancer types. A more pressing issue is that these studies did not provide evidence for a global alteration of the piRNA population in cancer versus normal cells; rather they focused on one or 2 specific piRNAs which may or may not be indicative of the entire piRNA population. There is no explanation of general piRNA changes which may be occurring, and no indication of what criteria were used to select individual piRNAs for further analysis. There might be important changes occurring in the piRNA population as a whole in cancer versus normal tissues. In order to more fully explore the potential role that piRNAs may play in cancer, it will be critical to examine global piRNA alterations in cancer tissues. As a first step, it would be interesting to do a global bioinformatic profile of piRNAs in cancer versus normal tissues, just as has been done for miRNA profiles in cancer [133]. Perhaps in this way, we will be able to develop a piRNA "signature" which differentiates cancer versus normal $[134,135]$. Importantly, because no mechanistic insight into the role of piRNAs in cancer has been explored, it is not known if piRNAs might be driving tumorigenesis or if their alteration is merely a bystander effect that is part of the cell's stem cell recapitulation process. Our data suggest that, because they are present even in parental MSCs, they are not driving the tumorigenic process. An important next step to identifying the functional role of piRNAs in cancer might be to perform transfection experiments with piRNAs of interest (or their antagoPIRs) and then monitor for tumorigenic growth (perhaps via colony forming or invasion assays). The current limitation is the ability to choose a piRNA or set of piRNAs of interest to exogenously express in vitro, since so little is known about the piRNAs, both globally and individually. 
piRNAs have also been identified in HeLa cells [81]. Moreover, Hili, a human Piwi homolog, has also been identified in HeLa cells and its up-regulation results in decreased Line1 transposon expression and decreased expression of piRNAs that are derived from Line1 repeat sequences. Importantly, the overall number of piRNAs did not change significantly when Hili was expressed-in line with our data which does not show a global shift in piRNA populations in response to Hiwi expression.

Based on data from a variety of model organisms where piRNA populations have been identified outside the germline, there may be a much wider expanse of piRNA populations across a variety of cell types, including both normal and cancer cells. A well-defined aspect of piRNAs is their association with Piwi family members, which have been found at high levels, developmentally, in normal germline stem cells and, aberrantly, in a variety of cancers. Importantly, though, the presence of piRNAs in the absence of Piwi homologs suggests that their roles in cells might be broader than previously thought. piRNAs may act with other proteins, perhaps members like their miRNA cousins, to exert their epigenetic silencing effects. Though Piwis are necessary for piRNA biogenesis, our work suggests that piRNAs may have a non-Piwi-related mechanism for biogenesis that has not yet been elucidated.

Our work opens the possibility that piRNA populations are present in many cell types, but are only able to carry out their epigenetic silencing effects when there is also high expression of Piwi orthologs, such as in normal germline stem cells and in cancer cells. In this way, piRNA expression, along with abundant Piwi expression, in germline stem cells results in normal transposon silencing during spermatogenesis via DNA methylation (Figure 5.1A). The cells develop normally into somatic tissues in which Piwis are absent (Figure 5.1B). However, in a cancer context -- where Hiwi is aberrantly highly expressed-the presence of both Piwi and piRNAs results in aberrant DNA methylation and over-silencing of genomic regions (perhaps 
including regions of tumor suppressor genes), which results in consequent tumorigenesis (Figure 5.1C) [136]. However, this model is highly speculative, as the exact role of piRNAs in cancer, and the mechanism by which they may be performing that role, remains largely unexplored both in cancer cell lines and in cancer tissues. In order to further test this model, it will be critical to determine the exact mechanism(s) by which piRNAs exert their gene silencing effects. Even in normal developmental systems, where piRNAs are best studied, a detailed mechanism of piRNA function remains elusive. For example, it is unknown whether piRNAs are responsible for gene regulation (not just transposon repeats). This remains especially understudied in a cancer context. It will also be enlightening to know whether piRNAs are associated with any chromatin modifying proteins, such as DNMTs, histone methyltransferases, or histone acetyltransferases. Additionally, Piwi proteins have been found to be associated with chromatin modifying proteins and with the chromatin itself [137]. This Piwi-chromatin association was indirectly shown to be RNA dependent. While this work was done in Drosophila, it will be ideal to see if Hiwi co-localizes with any chromatin modifying proteins or with the chromatin itself in human cells. Additionally, a co-localization experiment of piRNAs with the chromatin in MSCs will be useful to determine if piRNAs associate directly on the chromatin. As investigators continue to resolve these outstanding issues, a model for piRNA function, both in the presence and in the absence of Piwi proteins, will become more clearly defined. 


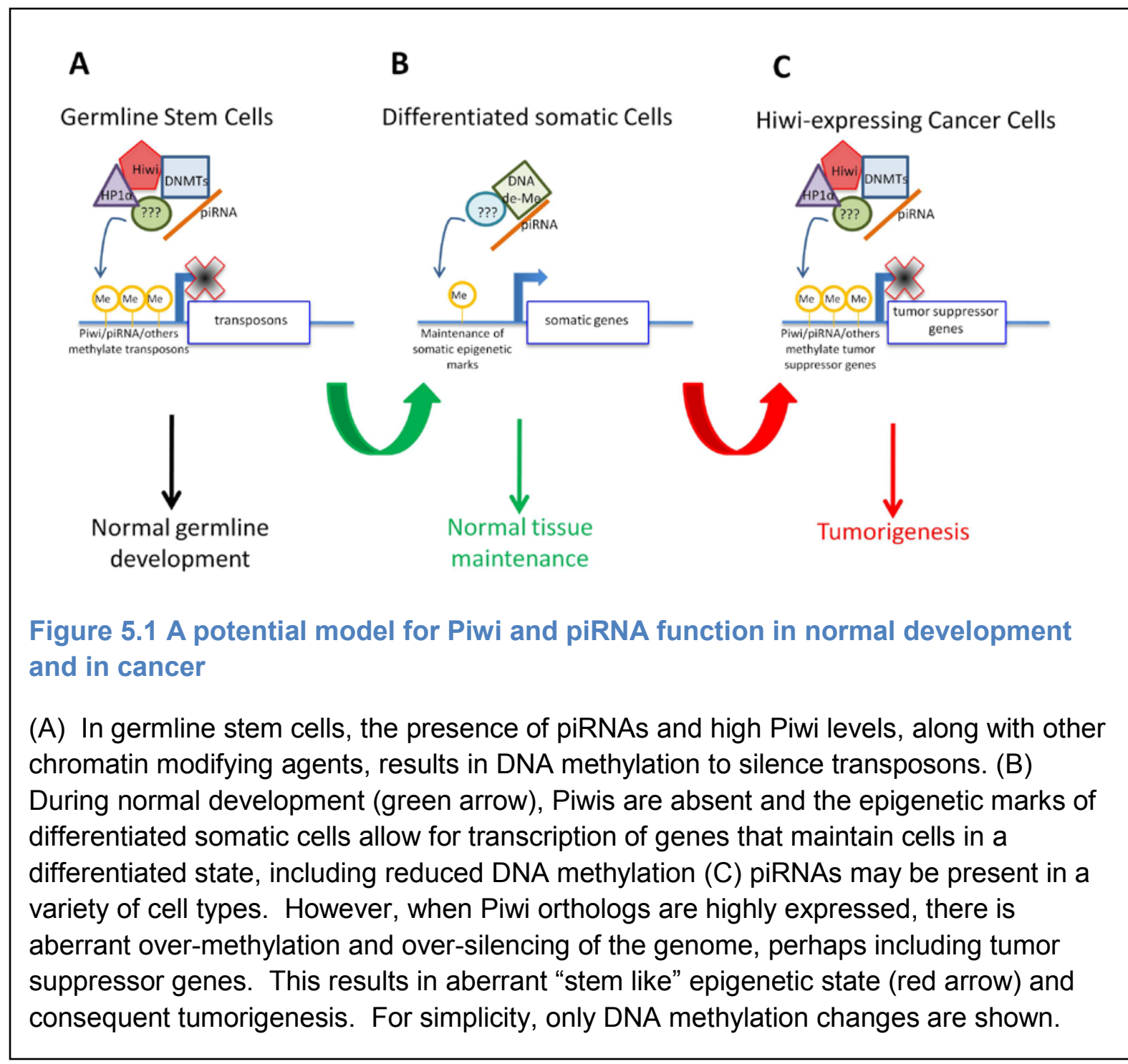

\section{Hiwi-mediated tumorigenesis is associated with DNA methylation}

Once we had identified a novel oncogenic role for Hiwi as a driver of tumorigenesis, we then sought to investigate a mechanism by which Hiwi mediates its sarcomagenic effects. In Chapter 4, we use Hiwi over-expression and knockdown models, along with human primary sarcoma samples, to show that global DNA methylation levels correlate with Hiwi, that DNA methyltransferases (DNMTs; DNMT1, DNMT3a, MDB2) are up-regulated in Hiwi-MSCs and that global DNA methylation increases are reversible upon treatment with a DNA de-methylating 
agent, 5-azacytidine. Moreover, 5-azacytidine treatment mimics knock down of Hiwi, in both decreased colony forming capacity and in the gene expression changes that occur. To further elucidate a DNA methylation-based mechanism for Hiwi-mediated tumorigenesis, we used gene expression overlapping to identify tumor suppressor genes which are altered in response to Hiwi expression and are also methylation dependent. In particular, we identified cell cycle dependent kinase inhibitors (CDKIs; p21, p27,p15) as having a tight inverse correlation with Hiwi expression in human sarcoma samples. CDKIs are a known class of tumor suppressors, as they prevent the cell from entering the cell cycle at cell cycle checkpoints when DNA damage has occurred $[138,139,140]$. Moreover, CDKIs are thought to be amenable to epigenetic regulation, especially in cancers such as ovarian cancer, leukemia, and lymphoma $[141,142,143]$. Since many cancers show an increase in methylation of CDKIs as an epigenetic mechanism for their down-regulation in cancer, CDKIs are likely a subset of tumor suppressor genes which are affected by Hiwi-associated DNA methylation and tumorigenesis.

We also investigated the promoter CpG methylation status of doxycycline-induced sh-HiwiMFH and find that promoter methylation does not shift globally upon Hiwi down-regulation. Moreover, bisulfite sequencing of the Rb1 and Line1 promoters shows no significant changes in promoter methylation as Hiwi levels change. Taken together, our data reveal that Hiwimediated tumorigenesis is associated with DNA methylation increase, though not at CpG promoter regions. This results in silencing of tumor suppressor genes, perhaps including CDKIs, and consequent tumorigenesis. Our data also suggest a novel therapeutic avenue for epigenetic agents in the treatment of Hiwi-expressing cancers.

Despite the tight correlations between Hiwi expression, global DNA methylation, and tumorigenesis described here, there are still several open questions which merit further investigation. The first is the determination of the exact cellular location of Hiwi. In our models and others $[73,121]$ Hiwi is predominantly cytoplasmic. However, its association with increased 
DNA methylation suggests that it is influencing nuclear proteins as well. We have thus far been unable to identify Hiwi localized to the nucleus. While we are limited by the number of commercially available antibodies for Hiwi, we have tried both immunohistochemical and western blotting approaches to identify nuclear Hiwi. However, others have also identified cytoplasmic Piwi homolog expression, so we believe our results are not simply due to technical difficulty. In one model, Hiwi may be shuttled in and out of the nucleus, although a majority remains in the cytoplasm and that is what has been detected thus far. Another model is that Hiwi uses intermediary proteins (such as DNMTs) to exert its DNA methylation-based silencing effects. In order to test the shuttling hypothesis, perhaps a fluorescent-tagged Hiwi can be transfected into cells and then followed so that its localization can be tracked via live microscopy from the cytoplasm to the nucleus. The association of Piwi homologs with chromatin modifying proteins has been examined by others $[137,144]$. Thus it would not be totally surprising if Hiwi also associated with a DNMT protein. However, this must be experimentally determined, such as through an IP-mass spectrometry experiment, where Hiwi and its associated proteins are pulled out and the Hiwi-associated complex is identified via a mass spectrometry approach. We have begun experiments to identify Hiwi binding partners using a yeast 2-hybrid approach.

We have shown that DNMT1, DNMT3a and MDB2 are up-regulated in Hiwi-MSCs, but they are up-regulated translationally; their RNA levels do not change in either Hiwi-MSCs (compared to parental MSCs) or in sh-Hiwi-MFH sarcoma cells (either before or after doxycycline induction to knockdown Hiwi levels). The exact mechanism by which high levels of Hiwi expression may promote translational up-regulation of DNMTs is still unclear. However, DNMTs are known to be regulated by post-transcriptional modifications and by small RNAs [145], so it is plausible that Hiwi promotes the post-transcriptional regulation of DNMTs. Our data, along with work from others which shows that Miwi (murine Piwi ortholog) interacts with ribosomes [122], suggest that Hiwi-associated DNA methylation likely occurs through regulation of DNMT 
translation. In order to more fully explore a mechanism of Hiwi-associated translational upregulation of DNMTs, an assessment of the stabilizing post-translational modifications of DNMTs should be done. This includes acetylation and de-ubiquitination [146], although other stabilizing post-translational modifications might also be regulating DNMTs in our Hiwi-MSCs compared to parental control MSCs. We are continuing to investigate the exact relationship between Hiwi and its epigenetic mechanisms in a cancer context, and many other laboratories are investigating this relationship in a developmental context $[2,28,29,30,36,55,58,59,60]$.

The relationship between Piwi orthologs and DNA methylation has been better studied in mice $[55,59]$, yet the exact mechanism of Piwi-mediated DNA methylation during development is still unclear. Our data showing that although global DNA methylation correlates with Hiwi levels, promoter $\mathrm{CpG}$ methylation does not change during Hiwi down-regulation, suggesting that Hiwi-associated DNA-methylation occurs globally at non-CpG promoter areas and/or at repetitive element regions. Confirming this interpretation, our bisulfite sequencing of either the Line1 element or the Rb1 promoter shows no change in DNA methylation at those sites as Hiwi levels change. Repetitive element methylation has previously been reported to result in long distance silencing via chromatin remodeling $[123,124]$. Since Piwis are thought to associate with piRNAs that have homology to repetitive elements $[61,125]$, this may explain why promoter methylation is not altered as Hiwi expression is altered in our systems.

Recently, Piwi homolog, Hiwi2, was implicated in p16 silencing through histone methylation rather than gene promoter methylation [119]. We then examined a number of histone methylation marks in our sh-Hiwi-MFH cells via immunofluorescence (IF) but found no alteration of these chromatin marks. Although we agree that promoters of silenced genes will display associated repressive chromatin marks, in our studies, we have been unable to identify these changes (at neither promoter DNA nor histone methylation). As an initial screen, we examined multiple histone methylation marks with an IF approach. Lacking a lead methylation mark to 
further investigate, we did not continue this line of investigation with other experimental approaches. However, a more exhaustive screen of all chromatin marks (histone acetylation, phosphorylation), not just histone methylation, might yield additional information regarding Hiwimediated epigenetic mechanisms of gene silencing, since it is thought that many chromatin modifying marks work in concert to induce chromatin changes [112]. The most common repressive chromatin changes, promoter methylation and the only one previously reported to be associated with Piwi orthologs, histone methylation, were not identified.

It is widely accepted that global DNA methylation levels are decreased in many human cancers, despite increases in specific CpG island methylation (at both promoter and nonpromoter regions). In this way, global hypo-methylation in cancer is thought to occur at repetitive elements, which make up a substantial portion of the genome (reviewed in $[128,129]$ ) Our data that Hiwi-associated DNA methylation increases as Hiwi expression increases is somewhat discordant with this generally accepted notion. However, Piwi homologs have a preference for silencing repetitive regions via DNA methylation during normal development, suggesting that Hiwi may target silencing of repetitive regions via DNA methylation in cancer cells, thus providing a mechanistic understanding of Hiwi's exception to the widely observed phenomenon of global hypo-methylation in cancer.

The role of Hiwi in adult neoplastic tissues remains fertile ground for further investigation, despite our studies which define a novel oncogenic role for Hiwi and reveal that tumor suppressor gene silencing via DNA methylation mechanistically accounts for this tumorigenesis. We further suggest that the use of epigenetic agents, such as 5-azacytidine, in the treatment of cancers which have high levels of Hiwi would be clinically beneficial. Because DNA demethylating agents are currently used to treat myelodysplastic disorders and acute myeloid leukemia [111], many issues regarding patient safety have already been addressed. So our suggestion that these epigenetic agents may also be clinically beneficial for Hiwi-expressing 
tumors is both reasonable and feasible. In fact, many clinical trials for DNA de-methylating agents in the treatment of a variety of diseases (including colorectal cancer, lymphoma, squamous cell carcinoma) are on-going.

\section{Model for Hiwi-mediated tumorigenesis}

Through in vitro and in vivo models of Hiwi over-expression and down-regulation, we have shown that Hiwi is a driver of tumorigenesis and is associated with global DNA hypermethylation. Importantly, this increase in DNA methylation occurs at non-promoter CpG regions and is reversible with DNA de-methylating agent treatment. A class of epigenetically-regulated tumor suppressor genes (CDKIs) has a tight inverse correlation with Hiwi in primary sarcomas. Synthesizing these mechanistic data, we can develop a working model in which Hiwi is aberrantly up-regulated in cancer, leading to an increase in global DNA methylation, perhaps through translational stabilization of DNMTs. This global DNA methylation silences nonpromoter CpG regions, including tumor suppressor genes such as CDKIs, resulting in Hiwimediated tumorigenesis. This model fits our data nicely, but there are still mechanistic details which need to be resolved if we are to generate a precise and detailed model of Hiwi-mediated tumorigenesis. One of those missing mechanistic details is the exact proteins which Hiwi is binding to in order to promote DNA methylation at non-promoter $\mathrm{CpG}$ regions.

In order to address this issue, we have undertaken a yeast 2-hybrid approach to identify any Hiwi binding partners (Dr. Katrin Rutschmann, Dualsystems Inc.). Hiwi was cloned into a bait vector and screened against a cDNA library generated from MFH sarcoma cells, the same cell line used in our doxycycline-induced sh-Hiwi-MFH model. The yeast 2 hybrid revealed only one Hiwi binding partner which was rescued multiple times (rescue multiple times is suggestive of a true protein-protein interaction). This binding partner is histone variant H3.3 (Figure 5.2). Histone H3.3 is replication independent and is associated with active transcription [147]. Our 
preliminary data suggesting Hiwi and H3.3 binding must still be independently confirmed using an immunoprecipitation-immunoblot (IP-IB) approach, since yeast 2-hybrid can be highly sensitive and thus physiologically irrelevant. Because commercially available Hiwi antibodies are not suitable for IP purposes, we have taken a tagging approach to these IP-IB experiments, using a HA-tagged Hiwi vector and a myc-tagged H3.3 vector. Co-transfection of these vectors into cells, then IP-IB for a Hiwi-H3.3 interaction using antibodies against the distinct molecular tags rather than the proteins directly is a straightforward method to circumvent a lack of appropriate commercial antibodies. IP-IB confirmatory experiments are on-going in our lab.

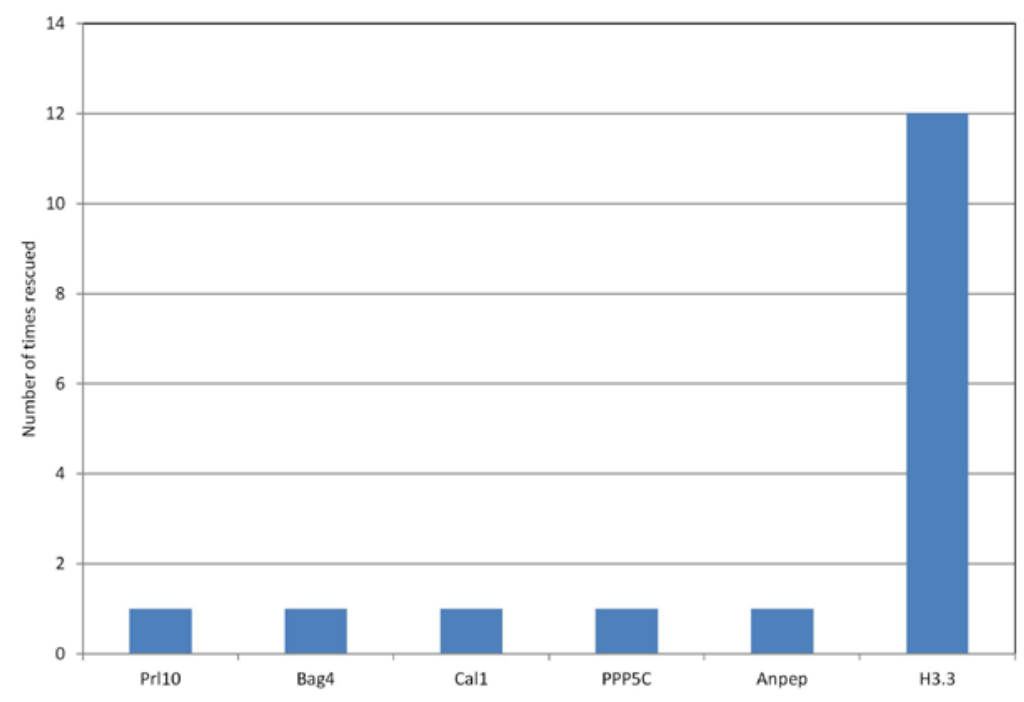

Figure 5.2 Yeast 2-hybrid results indicate H3.3 is a Hiwi binding partner

Histone variant $\mathrm{H} 3.3$ was the only protein to be rescued multiple times in the yeast 2hybrid assay, suggesting it is a true Hiwi-interactor. These data must be confirmed independently using a more physiological approach, such as immunoprecipitationimmunoblotting.

In light of these preliminary yeast 2-hybrid data indicating H3.3 is a Hiwi binding partner, we can update our working model for Hiwi-mediated tumorigenesis via DNA methylation to include H3.3 as a modulator of transcription (Figure 5.3A). In cancer cells which aberrantly 
express Hiwi at high levels, Hiwi directs DNA methylation, though this is limited to CpGs at nonpromoter regions. Hiwi interacts with $\mathrm{H} 3.3$, as the yeast 2-hybrid data suggest, at sites of active transcription in order to mediate transcriptional shut off. There are emerging data which suggest that $\mathrm{H} 3.3$, previously associated only with sites of active transcription, may also accumulate at silent loci, in both stem cells $[148,149]$ and in somatic cells [150]. It is possible then, that $\mathrm{H} 3.3$ enrichment is indicative of a poised chromatin state [151], rather than only active transcription. These data are in line with our model, which proposes that Hiwi expression is associated with silencing via DNA methylation. In our model, Hiwi may bind H3.3 at sites of poised transcription, thus encouraging transcriptional repression rather than activation.

Whether Hiwi recruits $\mathrm{H} 3.3$ to genomic regions which will be silenced or whether $\mathrm{H} 3.3$ is already present at baseline levels is still unknown, although our gene expression analyses find that H3.3 is present at very low baseline levels at MFH cells (But to the best of our knowledge, there has been no systematic investigation of $\mathrm{H} 3.3$ expression in primary human sarcomas). At the same time, Hiwi expression correlates with translational stabilization of DNMTs, further promoting silencing of the genome via DNA methylation. Translational stabilization of DNMTs has been established via de-acetylation and de-ubiquitination [146], including in a cancer context [152]. Since DNMTs were not found to be Hiwi binding partners via yeast 2-hybrid screening, it is likely that Hiwi promotes stabilization of DNMTs via intermediaries that interact with DNMTs. These might include deacetylases, ubiquitin proteases, and other stabilizing proteins which make up a multi-protein complex associated with the DNMT [153]. In this way, Hiwi may be able to stabilize DNMTs translationally without a direct interaction with DNMTs. The DNMTs then go on to methylate DNA at non-promoter CpG regions. Importantly, because promoter methylation status does not change, DNA methylation seems to be directed outside promoters, though its exact locations are still under investigation in our lab. It is possible that Hiwi is working, through DNMTs, to promote DNA methylation at non-promoter DNA locations through chromatin 
machinery that is local to the sites of methylation. Long distance epigenetic silencing is an established regulatory phenomenon in which methylation-based silencing occurs at large regions, rather than at discrete promoter sites [124]. Long distance methylation has been implicated in both normal development [154] and in cancers [155] and likely occurs in conjunction with local histone (de-) acetylases, histone methyltransferases and other epigenetic remodeling proteins to induce silencing of non-promoter CpG regions [155]. Methylation associated with long distance silencing may also occur regardless of the euchromatic or heterochromatic regions of the genes [123], in line with our data which suggest that Hiwi interacts with $\mathrm{H} 3.3$, a histone known to be at sites of poised chromatin. Recent work also suggests that in cancer, non-promoter CpGs may be more susceptible to methylation compared to normal cells [156]. In our model, due to increased DNA methylation, tumor suppressor genes are aberrantly silenced, which results in tumorigenesis. We specifically examined CDKIs and found them to be poorly expressed in Hiwi-expressing primary sarcomas. Our model is the first to examine an epigenetic mechanism regulating Hiwi-mediated tumorigenesis.

A remaining question in this working model is the exact location of Hiwi during the tumorigenic process. As mentioned earlier, our data and that from other groups [73,121], suggest that Hiwi is cytoplasmic. However, given the evidence that Hiwi is associated with DNA methylation, it is possible that Hiwi is shuttled into the nucleus, although we have been unable to consistently detect significant amounts of nuclear Hiwi in our IHC assays. Alternatively, cytoplasmic Hiwi might be using an intermediary (or set of intermediaries) to promote the stabilization of DNMTs (as described above). Stabilized DNMTs then go on to methylate DNA. Our yeast 2-hybrid data would suggest the former, although the 2 theories are not mutually exclusive. Further investigation of Hiwi localization might be undertaken using a western blot approach of Hiwi using nuclear and cytoplasmic separated cellular samples. An IF approach may also clarify Hiwi localization more precisely. We have tried both approaches and our results 
were limited by the availability of commercial antibodies appropriate for western blotting and IF. It may be necessary, therefore, to generate a new model for Hiwi over-expression, in which a tagged version of Hiwi is stably transfected/infected into sarcoma progenitor MSCs. By taking advantage of the wide availability of antibodies against tags (GFP, myc, flag, HA, etc), many experiments which are limited by Hiwi antibodies may be performed, including an IF analysis of Hiwi localization, protein-protein interactions and chromatin immunoprecipitations. A potential disadvantage of this approach is that the attached tag disrupts Hiwi's cellular function/localization. This risk can be minimized by using a tag that is relatively small, such as a flag tag. Similarly, a tagged transgenic mouse model for Hiwi over-expression may also be useful in order to track Hiwi expression during tumor development (This is discussed further in the following section.).

Our developing model for Hiwi mediated tumorigenesis suggests that Hiwi is a driver of tumorigenesis, via stabilization of DNMTs, and increased global DNA methylation. This results in silencing of tumor suppressor genes and results in tumorigenesis. Ours are the first data to elucidate an epigenetic mechanism for Hiwi-mediated tumorigenesis in a mammalian cancer context. 


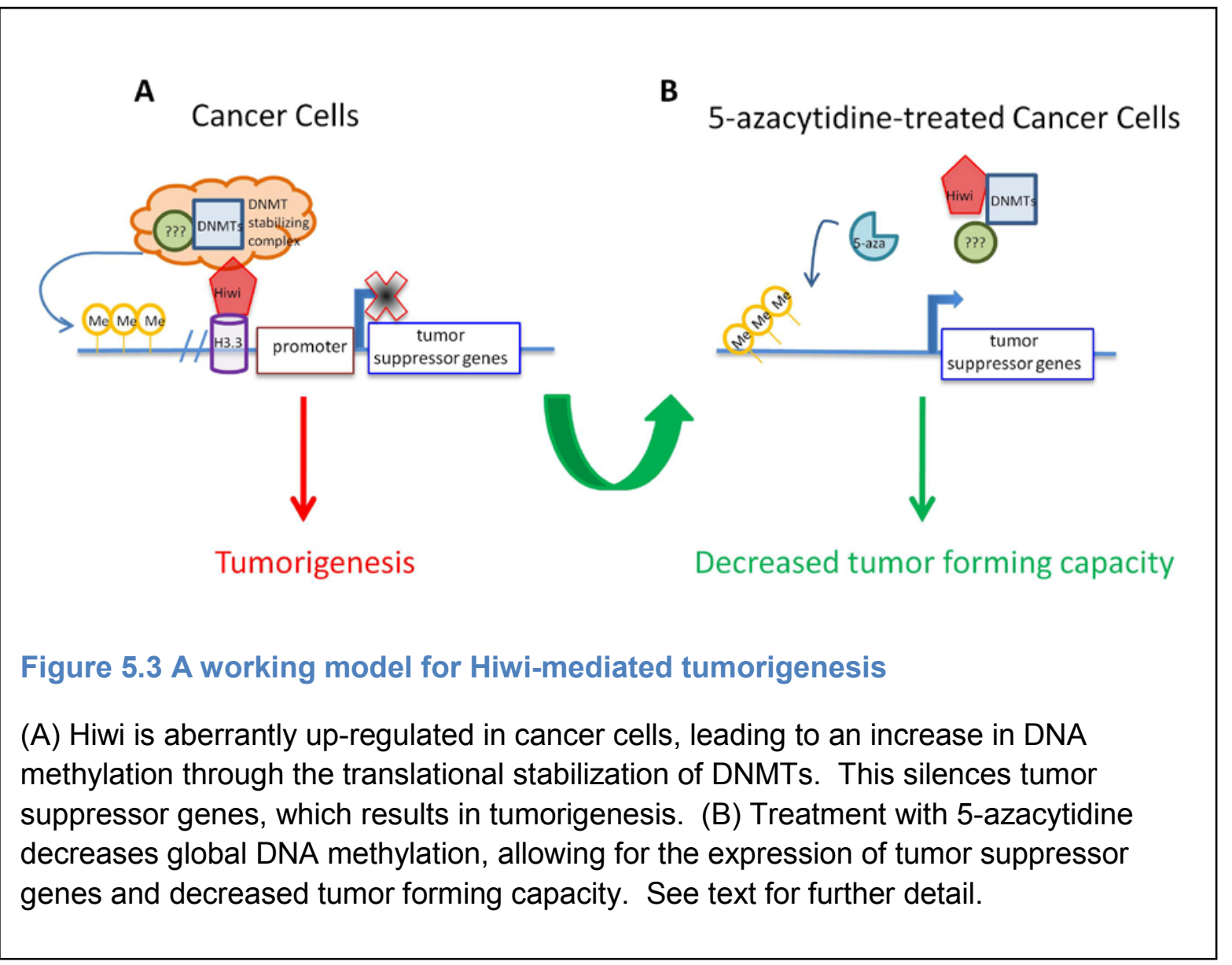

\section{Future Directions}

The next generation of transgenic mouse models of Hiwi over-expression

In Chapter 2, we describe the generation of an appendicular, mesoderm-specific transgenic mouse model of Hiwi over-expression. Because no relevant sarcoma mouse models have been generated to date, this was an important investigational tool which we developed. This model uses the Prx1 enhancer element to drive the expression of Hiwi in the developing limb bud mesoderm only. After careful monitoring and analysis of these mice for over 2 years, we find that the tumor incidence rate is below $10 \%$, although the tumors that do develop are in the expected distribution (on the limbs). 
One explanation for this low tumor incidence rate might be that Hiwi over-expression alone is not sufficient to form tumors at a high rate in a transgenic mouse model. Importantly, the Prx1 enhancer element has been used to specifically delete the Rb1 tumor suppressor gene in Prx1 tissues [92]. These Rb1-deleted mice do not form sarcomas at all and are ostensibly normal. In order to test the possibility that Hiwi over-expression, in combination with tumor suppressor gene loss, will result in a higher tumor incidence rate, we are currently generating Prx1-Hiwi+; Prx1-Rb1-/- mice. Given the aforementioned data on Prx-Rb1, we would expect that while Hiwi is sufficient to initiate sarcoma formation (albeit at a low rate), Rb is not. Rather, $\mathrm{Rb} 1$ might contribute to the acceleration of sarcoma formation or sarcoma maintenance, once another initiating mutation is introduced (described for Prx1-p53-/-;Prx1-Rb1-/- mice in [92]). In this way, we expect that Prx1-Hiwi+; Prx1-Rb1-/- mice will contain both initiating (Hiwi overexpression) and acceleration (Rb1 loss) events, which will result in an increased tumor incidence rate. This work is on-going in our lab. We anticipate this transgenic mouse model to be a more accurate recapitulation of human sarcomas than those currently used. This mouse model might also be useful in studying therapeutic avenues for sarcomas pre-clinically, since many human sarcomas have Rb1 mutations [157]. This, in combination with our data that find Hiwi to be highly expressed in human sarcomas, suggest that a Prx1-Hiwi+; Prx1-Rb-/transgenic mouse model would be highly relevant in recapitulating human sarcomas.

Another explanation of the low tumor incidence rate we find in our Prx1-Hiwi transgenic mouse model is that perhaps the promoter is simply too weak to drive the expression of Hiwi as robustly as necessary for Hiwi levels to reach a critical threshold beyond which tumors form. To assess this, we are currently generating a transgenic mouse model of Hiwi over-expression, where Hiwi is driven by a multisite pCAGG- promoter (targeted to the ROSA26 locus) which has floxed-stop alleles. Upon introduction of Prx1-Cre to the system (to ensure only mesodermallyderived tissues are affected), the floxed-stop is removed and Hiwi expression is driven by 
pCAGG, known to be 8 to 10 fold stronger than the pROSA26 promoter, based on transgene expression [158]. Additionally, the multisite pCAGG vector contains a GFP tag C-terminal to the Hiwi cDNA, as a method to visualize Hiwi expression in any tumors which develop. Using this approach, we hope to generate mesoderm-specific Hiwi -over-expression in our mouse model that is much higher than that of the Prx1-Hiwi mouse model. This work is continuing in our lab.

\section{A novel therapeutic avenue for Hiwi-expressing tumors}

In Chapter 3, we observe that 5-azacytidine treatment is able to reverse the increased global DNA methylation found in Hiwi-MSCs. Moreover, 5-azacytidine-treated sh-Hiwi MFH cells show decreased colony formation capacity that is very similar to what is seen during doxycycline-induced knock down of Hiwi. We go on to show that the gene expression changes of both 5-azacytidine treated cells and doxycycline-induced Hiwi knock down cells are very similar, with a high degree of overlapping gene changes. These data suggest that 5-azacytidine treatment and Hiwi knock down might be working through similar mechanisms to reduce the colony forming capacity of MFH sarcoma cells. Because 5-azacytidine has already been used for treatment of acute myeloid leukemia and myelodysplastic syndromes [111], our suggestion that 5-azacytidine may also be used to treat Hiwi-expressing cancers is clinically feasible, since toxicity issues have already been vetted. Importantly, though, 5-azacytidine in the treatment of Hiwi-expressing cancers might be beneficial at lower doses which modify DNA methylation rather than the higher doses used in myelodysplastic syndrome treatment which induce cell death [112].

We are able to integrate these data into our working model of Hiwi-mediated tumorigenesis: treatment with 5-azacytidine decreases global DNA methylation levels and tumor suppressor gene expression is restored, resulting in decreased in tumor forming capacity 
(Figure 5.3B). In both colony formation and in gene expression profiles, treatment of sarcoma MFH cells with 5-azacytidine seems to mimic Hiwi knock down in sarcoma MFH cells. This leads us to suggest that the use of DNA de-methylating agents might be clinically relevant to treating cancers. Tumors which express Hiwi at high levels (sarcomas, but also a variety of other cancers $[65,66,67,69,95])$ might be particularly sensitive to treatment with epigenetic agents such as 5-azacytidine. Just as Her2 expression is used as a marker for identifying breast cancers which are might be particularly sensitive to Herceptin treatment [159], Hiwi expression might also be used as a marker to identify cancers which would be most sensitive to epigenetic therapies. Our data are the first to suggest a therapeutic rationale for the use of epigenetic agents in the treatment of Hiwi-expressing cancers.

\section{Potential global implications of our studies}

Work from a variety of labs implicates Piwi family proteins in the silencing of transposon repeats during normal development of the germline. At the same time, Hiwi, the human homolog of Piwi, has been found to be elevated in a variety of cancers, including sarcomas. However, a potential connection between Hiwi and sarcomagenesis remained unexplored before we started the studies described here. We have demonstrated for the first time a causative oncogenic role for Hiwi as a driver of sarcomagenesis. Moreover, we have found that Hiwi levels correlate with global DNA methylation levels and DNMT expression levels. Hiwi has a tight inverse correlation with a class of tumor suppressor genes, cell cycle dependent kinase inhibitors (CDKIs). These data are the first to implicate DNMTs in the mechanism behind Hiwimediated DNA methylation and silencing, either in normal development or in cancer. Our studies open a new direction for the study of the exact role of DNMTs in the epigenetic mechanisms behind Hiwi-mediated tumorigenesis and also Hiwi-mediated transposon silencing in the normal germline. These data also implicate non-promoter $\mathrm{CpG}$ methylation as a regulator of gene expression, at least of the CDKIs examined here. Because we are the first group to 
identify tumor suppressor genes which are aberrantly regulated by Hiwi expression (although indirectly), we have established the first set of genes, rather than repetitive elements, which are sensitive to Hiwi expression. Together, our data suggest a mechanism for Hiwi-mediated tumorigenesis in which Hiwi promotes DNA methylation and tumor suppressor gene silencing. Ours are the first reports to provide mechanistic insight into Hiwi's role in human cancers. Ours are also the first reports to suggest a therapeutic rationale for clinically treating Hiwi-expressing cancers with epigenetic agents such as 5-azacytidine.

Aside from revealing an oncogenic role for Hiwi and its mechanistic basis in human cancers, we have also identified piRNAs in our MSCs, in the absence of Piwi homolog expression. Our data are in line with that from other groups which suggest that piRNAs may be expressed much more broadly than previously thought. piRNAs have been found in many tissues outside the germline, including in some human cancers. Our data also have implications for the field of piRNA biogenesis. Without Piwi expression, piRNAs may use some alternative non-ping-pong based generation mechanism, but what this might be remains unexplored. Alternatively, there may be a novel Piwi homolog that has yet to be identified and this Piwi homolog is mediating piRNA biogenesis in MSCs. Additionally, the majority of piRNAs we identified do not map to gene regions, which is in keeping with the known homology of piRNAs in a variety of model organisms to repetitive regions rather than genes.

Taken together, our data underscore the importance of Hiwi expression as a driver of tumorigenesis and reveal mechanistically that Hiwi-associated DNA methylation silences tumor suppressor genes to mediate tumorigenesis. Our work has implications for the basic biology of Piwi protein/piRNA function, for the epigenetic control of gene expression and also for cancer biology and development of targeted therapeutics. 


\section{References}

1. Charytonowicz E, Cordon-Cardo C, Matushansky I, Ziman M (2009) Alveolar rhabdomyosarcoma: is the cell of origin a mesenchymal stem cell? Cancer Lett 279: 126-136.

2. Seto AG, Kingston RE, Lau NC (2007) The coming of age for Piwi proteins. Mol Cell 26: 603-609.

3. Kim VN, Han J, Siomi MC (2009) Biogenesis of small RNAs in animals. Nat Rev Mol Cell Biol 10: 126139.

4. Krampera M, Pizzolo G, Aprili G, Franchini M (2006) Mesenchymal stem cells for bone, cartilage, tendon and skeletal muscle repair. Bone 39: 678-683.

5. Zuk PA, Zhu M, Ashjian P, De Ugarte DA, Huang Jl, et al. (2002) Human adipose tissue is a source of multipotent stem cells. Mol Biol Cell 13: 4279-4295.

6. Pittenger MF, Mackay AM, Beck SC, Jaiswal RK, Douglas R, et al. (1999) Multilineage potential of adult human mesenchymal stem cells. Science 284: 143-147.

7. Smith JR, Pochampally R, Perry A, Hsu SC, Prockop DJ (2004) Isolation of a highly clonogenic and multipotential subfraction of adult stem cells from bone marrow stroma. Stem Cells 22: 823831.

8. Murphey MD (2007) World Health Organization classification of bone and soft tissue tumors: modifications and implications for radiologists. Semin Musculoskelet Radiol 11: 201-214.

9. Matushansky I, Hernando E, Socci ND, Mills JE, Matos TA, et al. (2007) Derivation of sarcomas from mesenchymal stem cells via inactivation of the Wnt pathway. J Clin Invest 117: 3248-3257.

10. Matushansky I, Hernando E, Socci ND, Matos T, Mills J, et al. (2008) A developmental model of sarcomagenesis defines a differentiation-based classification for liposarcomas. Am J Pathol 172: 1069-1080.

11. DeVita VT (2008) Cancer: Principles \& Practice of Oncology: Lippincott Williams \& Wilkins.

12. Haydon RC, Luu HH, He TC (2007) Osteosarcoma and osteoblastic differentiation: a new perspective on oncogenesis. Clin Orthop Relat Res 454: 237-246.

13. Tang N, Song WX, Luo J, Haydon RC, He TC (2008) Osteosarcoma development and stem cell differentiation. Clin Orthop Relat Res 466: 2114-2130.

14. Kansara M, Thomas DM (2007) Molecular pathogenesis of osteosarcoma. DNA Cell Biol 26: 1-18.

15. Jain M, Arvanitis C, Chu K, Dewey W, Leonhardt E, et al. (2002) Sustained loss of a neoplastic phenotype by brief inactivation of MYC. Science 297: 102-104.

16. Dick JE (2008) Stem cell concepts renew cancer research. Blood 112: 4793-4807.

17. Orfao A, Schmitz G, Brando B, Ruiz-Arguelles A, Basso G, et al. (1999) Clinically useful information provided by the flow cytometric immunophenotyping of hematological malignancies: current status and future directions. Clin Chem 45: 1708-1717.

18. Krivtsov AV, Twomey D, Feng Z, Stubbs MC, Wang Y, et al. (2006) Transformation from committed progenitor to leukaemia stem cell initiated by MLL-AF9. Nature 442: 818-822.

19. Feinberg AP, Vogelstein B (1983) Hypomethylation distinguishes genes of some human cancers from their normal counterparts. Nature 301: 89-92.

20. Hettmer S, Liu J, Miller CM, Lindsay MC, Sparks CA, et al. (2011) Sarcomas induced in discrete subsets of prospectively isolated skeletal muscle cells. Proc Natl Acad Sci U S A 108: 2000220007.

21. Choi J, Curtis SJ, Roy DM, Flesken-Nikitin A, Nikitin AY (2010) Local mesenchymal stem/progenitor cells are a preferential target for initiation of adult soft tissue sarcomas associated with p53 and $\mathrm{Rb}$ deficiency. Am J Pathol 177: 2645-2658. 
22. Riggi N, Suva ML, De Vito C, Provero P, Stehle JC, et al. (2010) EWS-FLI-1 modulates miRNA145 and SOX2 expression to initiate mesenchymal stem cell reprogramming toward Ewing sarcoma cancer stem cells. Genes Dev 24: 916-932.

23. Sadikovic B, Andrews J, Rodenhiser DI (2007) DNA methylation analysis using CpG microarrays is impaired in benzopyrene exposed cells. Toxicol Appl Pharmacol 225: 300-309.

24. Krishnan V, Bryant HU, Macdougald OA (2006) Regulation of bone mass by Wnt signaling. J Clin Invest 116: 1202-1209.

25. Ross SE, Hemati N, Longo KA, Bennett CN, Lucas PC, et al. (2000) Inhibition of adipogenesis by Wnt signaling. Science 289: 950-953.

26. Taubert H, Greither T, Kaushal D, Wurl P, Bache M, et al. (2007) Expression of the stem cell selfrenewal gene Hiwi and risk of tumour-related death in patients with soft-tissue sarcoma. Oncogene 26: 1098-1100.

27. Taubert H, Wurl P, Greither T, Kappler M, Bache M, et al. (2007) Stem cell-associated genes are extremely poor prognostic factors for soft-tissue sarcoma patients. Oncogene 26: 7170-7174.

28. Sharma AK, Nelson MC, Brandt JE, Wessman M, Mahmud N, et al. (2001) Human CD34(+) stem cells express the hiwi gene, a human homologue of the Drosophila gene piwi. Blood 97: 426-434.

29. O'Donnell KA, Boeke JD (2007) Mighty Piwis defend the germline against genome intruders. Cell 129: 37-44.

30. Lin $\mathrm{H}$ (2007) piRNAs in the germ line. Science 316: 397.

31. Lin H, Spradling AC (1997) A novel group of pumilio mutations affects the asymmetric division of germline stem cells in the Drosophila ovary. Development 124: 2463-2476.

32. Cox DN, Chao A, Baker J, Chang L, Qiao D, et al. (1998) A novel class of evolutionarily conserved genes defined by piwi are essential for stem cell self-renewal. Genes Dev 12: 3715-3727.

33. Cox DN, Chao A, Lin H (2000) piwi encodes a nucleoplasmic factor whose activity modulates the number and division rate of germline stem cells. Development 127: 503-514.

34. Gunawardane LS, Saito K, Nishida KM, Miyoshi K, Kawamura Y, et al. (2007) A slicer-mediated mechanism for repeat-associated siRNA 5' end formation in Drosophila. Science 315: 1587-1590.

35. Saito K, Nishida KM, Mori T, Kawamura Y, Miyoshi K, et al. (2006) Specific association of Piwi with rasiRNAs derived from retrotransposon and heterochromatic regions in the Drosophila genome. Genes Dev 20: 2214-2222.

36. Brennecke J, Aravin AA, Stark A, Dus M, Kellis M, et al. (2007) Discrete small RNA-generating loci as master regulators of transposon activity in Drosophila. Cell 128: 1089-1103.

37. Houwing S, Kamminga LM, Berezikov E, Cronembold D, Girard A, et al. (2007) A role for Piwi and piRNAs in germ cell maintenance and transposon silencing in Zebrafish. Cell 129: 69-82.

38. Sun H, Li D, Chen S, Liu Y, Liao X, et al. (2010) Zili inhibits transforming growth factor-beta signaling by interacting with Smad4. J Biol Chem 285: 4243-4250.

39. Lynn K, Fernandez A, Aida M, Sedbrook J, Tasaka M, et al. (1999) The PINHEAD/ZWILLE gene acts pleiotropically in Arabidopsis development and has overlapping functions with the ARGONAUTE1 gene. Development 126: 469-481.

40. Moussian B, Haecker A, Laux T (2003) ZWILLE buffers meristem stability in Arabidopsis thaliana. Dev Genes Evol 213: 534-540.

41. Moussian B, Schoof H, Haecker A, Jurgens G, Laux T (1998) Role of the ZWILLE gene in the regulation of central shoot meristem cell fate during Arabidopsis embryogenesis. Embo J 17: 1799-1809.

42. Palakodeti D, Smielewska M, Lu YC, Yeo GW, Graveley BR (2008) The PIWI proteins SMEDWI-2 and SMEDWI-3 are required for stem cell function and piRNA expression in planarians. Rna 14: 11741186. 
43. Reddien PW, Oviedo NJ, Jennings JR, Jenkin JC, Sanchez Alvarado A (2005) SMEDWI-2 is a PIWI-like protein that regulates planarian stem cells. Science 310: 1327-1330.

44. Kuramochi-Miyagawa S, Kimura T, Ijiri TW, Isobe T, Asada N, et al. (2004) Mili, a mammalian member of piwi family gene, is essential for spermatogenesis. Development 131: 839-849.

45. Kuramochi-Miyagawa S, Watanabe T, Gotoh K, Totoki Y, Toyoda A, et al. (2008) DNA methylation of retrotransposon genes is regulated by Piwi family members MILI and MIWI2 in murine fetal testes. Genes Dev 22: 908-917.

46. Aravin AA, Lagos-Quintana M, Yalcin A, Zavolan M, Marks D, et al. (2003) The small RNA profile during Drosophila melanogaster development. Dev Cell 5: 337-350.

47. Horwich MD, Li C, Matranga C, Vagin V, Farley G, et al. (2007) The Drosophila RNA methyltransferase, DmHen1, modifies germline piRNAs and single-stranded siRNAs in RISC. Curr Biol 17: 1265-1272.

48. Kirino Y, Mourelatos Z (2007) 2'-O-methyl modification in mouse piRNAs and its methylase. Nucleic Acids Symp Ser (Oxf): 417-418.

49. Kirino Y, Mourelatos Z (2007) Mouse Piwi-interacting RNAs are 2'-O-methylated at their 3' termini. Nat Struct Mol Biol 14: 347-348.

50. Ohara T, Sakaguchi Y, Suzuki T, Ueda H, Miyauchi K, et al. (2007) The 3' termini of mouse Piwiinteracting RNAs are 2'-O-methylated. Nat Struct Mol Biol 14: 349-350.

51. Saito K, Sakaguchi Y, Suzuki T, Suzuki T, Siomi H, et al. (2007) Pimet, the Drosophila homolog of HEN1, mediates 2'-O-methylation of Piwi- interacting RNAs at their 3' ends. Genes Dev 21: 16031608.

52. Vagin VV, Sigova A, Li C, Seitz H, Gvozdev V, et al. (2006) A distinct small RNA pathway silences selfish genetic elements in the germline. Science 313: 320-324.

53. Li C, Vagin VV, Lee S, Xu J, Ma S, et al. (2009) Collapse of germline piRNAs in the absence of Argonaute3 reveals somatic piRNAs in flies. Cell 137: 509-521.

54. Aravin A, Gaidatzis D, Pfeffer S, Lagos-Quintana M, Landgraf P, et al. (2006) A novel class of small RNAs bind to MILI protein in mouse testes. Nature 442: 203-207.

55. Aravin AA, Sachidanandam R, Bourc'his D, Schaefer C, Pezic D, et al. (2008) A piRNA pathway primed by individual transposons is linked to de novo DNA methylation in mice. Mol Cell 31: 785-799.

56. Girard A, Sachidanandam R, Hannon GJ, Carmell MA (2006) A germline-specific class of small RNAs binds mammalian Piwi proteins. Nature 442: 199-202.

57. Grivna ST, Beyret E, Wang Z, Lin H (2006) A novel class of small RNAs in mouse spermatogenic cells. Genes Dev 20: 1709-1714.

58. Aravin AA, Hannon GJ, Brennecke J (2007) The Piwi-piRNA pathway provides an adaptive defense in the transposon arms race. Science 318: 761-764.

59. Carmell MA, Girard A, van de Kant HJ, Bourc'his D, Bestor TH, et al. (2007) MIWI2 is essential for spermatogenesis and repression of transposons in the mouse male germline. Dev Cell 12: 503514.

60. Reuter M, Chuma S, Tanaka T, Franz T, Stark A, et al. (2009) Loss of the Mili-interacting Tudor domain-containing protein-1 activates transposons and alters the Mili-associated small RNA profile. Nat Struct Mol Biol 16: 639-646.

61. Siomi MC, Sato K, Pezic D, Aravin AA (2011) PIWI-interacting small RNAs: the vanguard of genome defence. Nat Rev Mol Cell Biol 12: 246-258.

62. Yin H, Lin H (2007) An epigenetic activation role of Piwi and a Piwi-associated piRNA in Drosophila melanogaster. Nature 450: 304-308.

63. Lin H, Yin H (2008) A novel epigenetic mechanism in Drosophila somatic cells mediated by Piwi and piRNAs. Cold Spring Harb Symp Quant Biol 73: 273-281. 
64. Qiao D, Zeeman AM, Deng W, Looijenga LH, Lin H (2002) Molecular characterization of hiwi, a human member of the piwi gene family whose overexpression is correlated to seminomas. Oncogene 21: 3988-3999.

65. Sun G, Wang Y, Sun L, Luo H, Liu N, et al. (2011) Clinical significance of Hiwi gene expression in gliomas. Brain Res 1373: 183-188.

66. He W, Wang Z, Wang Q, Fan Q, Shou C, et al. (2009) Expression of HIWI in human esophageal squamous cell carcinoma is significantly associated with poorer prognosis. BMC Cancer 9: 426.

67. Grochola LF, Greither T, Taubert H, Moller P, Knippschild U, et al. (2008) The stem cell-associated Hiwi gene in human adenocarcinoma of the pancreas: expression and risk of tumour-related death. Br J Cancer 99: 1083-1088.

68. Jiang J, Zhang H, Tang Q, Hao B, Shi R (2011) Expression of HIWI in human hepatocellular carcinoma. Cell Biochem Biophys 61: 53-58.

69. Liu X, Sun Y, Guo J, Ma H, Li J, et al. (2006) Expression of hiwi gene in human gastric cancer was associated with proliferation of cancer cells. Int J Cancer 118: 1922-1929.

70. Watanabe T, Takeda A, Tsukiyama T, Mise K, Okuno T, et al. (2006) Identification and characterization of two novel classes of small RNAs in the mouse germline: retrotransposonderived siRNAs in oocytes and germline small RNAs in testes. Genes Dev 20: 1732-1743.

71. Tan CH, Lee TC, Weeraratne SD, Korzh V, Lim TM, et al. (2002) Ziwi, the zebrafish homologue of the Drosophila piwi: co-localization with vasa at the embryonic genital ridge and gonad-specific expression in the adults. Gene Expr Patterns 2: 257-260.

72. Kim VN (2006) Small RNAs just got bigger: Piwi-interacting RNAs (piRNAs) in mammalian testes. Genes Dev 20: 1993-1997.

73. Deng W, Lin H (2002) miwi, a murine homolog of piwi, encodes a cytoplasmic protein essential for spermatogenesis. Dev Cell 2: 819-830.

74. Tushir JS, Zamore PD, Zhang Z (2009) SnapShot: mouse piRNAs, PIWI proteins, and the ping-pong cycle. Cell 139: 830-830 e831.

75. Beyret $\mathrm{E}$, Lin $\mathrm{H}$ (2011) Pinpointing the expression of piRNAs and function of the PIWI protein subfamily during spermatogenesis in the mouse. Dev Biol 355: 215-226.

76. Wilczynska A, Minshall N, Armisen J, Miska EA, Standart N (2009) Two Piwi proteins, Xiwi and Xili, are expressed in the Xenopus female germline. RNA 15: 337-345.

77. Ishizu H, Nagao A, Siomi H (2011) Gatekeepers for Piwi-piRNA complexes to enter the nucleus. Curr Opin Genet Dev 21: 484-490.

78. Haase AD, Fenoglio S, Muerdter F, Guzzardo PM, Czech B, et al. (2010) Probing the initiation and effector phases of the somatic piRNA pathway in Drosophila. Genes Dev 24: 2499-2504.

79. Lee EJ, Banerjee $S$, Zhou H, Jammalamadaka A, Arcila M, et al. (2011) Identification of piRNAs in the central nervous system. RNA 17: 1090-1099.

80. Yan Z, Hu HY, Jiang X, Maierhofer V, Neb E, et al. (2011) Widespread expression of piRNA-like molecules in somatic tissues. Nucleic Acids Res.

81. Lu Y, Li C, Zhang K, Sun H, Tao D, et al. (2010) Identification of piRNAs in Hela cells by massive parallel sequencing. BMB Rep 43: 635-641.

82. Klattenhoff C, Theurkauf W (2008) Biogenesis and germline functions of piRNAs. Development 135: 3-9.

83. Wu Q, Ma Q, Shehadeh LA, Wilson A, Xia L, et al. (2010) Expression of the Argonaute protein PiwiL2 and piRNAs in adult mouse mesenchymal stem cells. Biochem Biophys Res Commun 396: 915920.

84. Corcoran C, Friel AM, Duffy MJ, Crown J, O'Driscoll L (2011) Intracellular and extracellular microRNAs in breast cancer. Clin Chem 57: 18-32. 
85. Coppola V, De Maria R, Bonci D (2010) MicroRNAs and prostate cancer. Endocr Relat Cancer 17: F117.

86. Huang $S$, He X (2011) The role of microRNAs in liver cancer progression. Br J Cancer 104: 235-240.

87. Jiang J, Zhang H, Tang Q, Hao B, Shi R (2011) Expression of HIWI in Human Hepatocellular Carcinoma. Cell Biochem Biophys.

88. Cheng J, Guo JM, Xiao BX, Miao Y, Jiang Z, et al. (2011) piRNA, the new non-coding RNA, is aberrantly expressed in human cancer cells. Clin Chim Acta 412: 1621-1625.

89. Cui L, Lou Y, Zhang X, Zhou H, Deng H, et al. (2011) Detection of circulating tumor cells in peripheral blood from patients with gastric cancer using piRNAs as markers. Clin Biochem.

90. Stojadinovic A, Leung DH, Allen P, Lewis JJ, Jaques DP, et al. (2002) Primary adult soft tissue sarcoma: time-dependent influence of prognostic variables. J Clin Oncol 20: 4344-4352.

91. Logan M, Martin JF, Nagy A, Lobe C, Olson EN, et al. (2002) Expression of Cre Recombinase in the developing mouse limb bud driven by a Prxl enhancer. Genesis 33: 77-80.

92. Lin PP, Pandey MK, Jin F, Raymond AK, Akiyama H, et al. (2009) Targeted mutation of p53 and Rb in mesenchymal cells of the limb bud produces sarcomas in mice. Carcinogenesis 30: 1789-1795.

93. Mills J, Matos T, Charytonowicz E, Hricik T, Castillo-Martin M, et al. (2009) Characterization and comparison of the properties of sarcoma cell lines in vitro and in vivo. Hum Cell 22: 85-93.

94. Mills J, Hricik T, Siddiqi S, Matushansky I (2011) Chromatin structure predicts epigenetic therapy responsiveness in sarcoma. Mol Cancer Ther.

95. Zhao YM, Zhou JM, Wang LR, He HW, Wang XL, et al. (2011) HIWI is associated with prognosis in patients with hepatocellular carcinoma after curative resection. Cancer.

96. Zeng Y, Qu LK, Meng L, Liu CY, Dong B, et al. (2011) HIWI expression profile in cancer cells and its prognostic value for patients with colorectal cancer. Chin Med J (Engl) 124: 2144-2149.

97. Cawthorne C, Swindell R, Stratford IJ, Dive C, Welman A (2007) Comparison of doxycycline delivery methods for Tet-inducible gene expression in a subcutaneous xenograft model. J Biomol Tech 18: $120-123$.

98. Jia XQ, Cheng HQ, Li H, Zhu Y, Li YH, et al. (2011) Inhibition of connective tissue growth factor overexpression decreases growth of hepatocellular carcinoma cells in vitro and in vivo. Chin Med J (Engl) 124: 3794-3799.

99. Wang L, Liu Z, Balivada S, Shrestha T, Bossmann S, et al. (2012) Interleukin-1beta and transforming growth factor-beta cooperate to induce neurosphere formation and increase tumorigenicity of adherent LN-229 glioma cells. Stem Cell Res Ther 3: 5.

100. Tushir JS, Zamore PD, Zhang Z (2009) SnapShot: Fly piRNAs, PIWI proteins, and the ping-pong cycle. Cell 139: 634, 634 e631.

101. Esposito T, Magliocca S, Formicola D, Gianfrancesco F (2011) piR_015520 belongs to Piwiassociated RNAs regulates expression of the human melatonin receptor $1 \mathrm{~A}$ gene. PLoS One 6: e22727.

102. Baba M, Hong SB, Sharma N, Warren MB, Nickerson ML, et al. (2006) Folliculin encoded by the BHD gene interacts with a binding protein, FNIP1, and AMPK, and is involved in AMPK and MTOR signaling. Proc Natl Acad Sci U S A 103: 15552-15557.

103. Xie Z, Zhang H, Tsai W, Zhang Y, Du Y, et al. (2008) Zinc finger protein ZBTB20 is a key repressor of alpha-fetoprotein gene transcription in liver. Proc Natl Acad Sci U S A 105: 10859-10864.

104. Sutherland AP, Zhang H, Zhang Y, Michaud M, Xie Z, et al. (2009) Zinc finger protein Zbtb20 is essential for postnatal survival and glucose homeostasis. Mol Cell Biol 29: 2804-2815.

105. Xie Z, Ma X, Ji W, Zhou G, Lu Y, et al. (2010) Zbtb20 is essential for the specification of CA1 field identity in the developing hippocampus. Proc Natl Acad Sci U S A 107: 6510-6515. 
106. Wang Q, Tan YX, Ren YB, Dong LW, Xie ZF, et al. (2011) Zinc finger protein ZBTB20 expression is increased in hepatocellular carcinoma and associated with poor prognosis. BMC Cancer 11: 271.

107. Krammer J, Digel M, Ehehalt F, Stremmel W, Fullekrug J, et al. (2011) Overexpression of CD36 and acyl-CoA synthetases FATP2, FATP4 and ACSL1 increases fatty acid uptake in human hepatoma cells. Int J Med Sci 8: 599-614.

108. Goei VL, Choi J, Ahn J, Bowlus CL, Raha-Chowdhury R, et al. (1998) Human gamma-aminobutyric acid $B$ receptor gene: complementary DNA cloning, expression, chromosomal location, and genomic organization. Biol Psychiatry 44: 659-666.

109. Li Y, Fu L, Wong AM, Fan YH, Li MX, et al. (2011) Identification of genes with allelic imbalance on $6 p$ associated with nasopharyngeal carcinoma in southern Chinese. PLoS One 6: e14562.

110. Koch P, Bohlmann I, Schafer M, Hansen-Hagge TE, Kiyoi H, et al. (2000) Identification of a novel putative Ran-binding protein and its close homologue. Biochem Biophys Res Commun 278: 241249.

111. Keating GM (2012) Azacitidine: A Review of its Use in the Management of Myelodysplastic Syndromes/Acute Myeloid Leukaemia. Drugs.

112. Siddiqi S, Mills J, Matushansky I (2010) Epigenetic remodeling of chromatin architecture: exploring tumor differentiation therapies in mesenchymal stem cells and sarcomas. Curr Stem Cell Res Ther 5: 63-73.

113. Mills J, Hricik T, Siddiqi S, Matushansky I (2011) Chromatin structure predicts epigenetic therapy responsiveness in sarcoma. Mol Cancer Ther 10: 313-324.

114. Baylin SB, Herman JG, Graff JR, Vertino PM, Issa JP (1998) Alterations in DNA methylation: a fundamental aspect of neoplasia. Adv Cancer Res 72: 141-196.

115. Costello JF, Fruhwald MC, Smiraglia DJ, Rush LJ, Robertson GP, et al. (2000) Aberrant CpG-island methylation has non-random and tumour-type-specific patterns. Nat Genet 24: 132-138.

116. Smiraglia DJ, Plass C (2002) The study of aberrant methylation in cancer via restriction landmark genomic scanning. Oncogene 21: 5414-5426.

117. Higgins ME, Claremont M, Major JE, Sander C, Lash AE (2007) CancerGenes: a gene selection resource for cancer genome projects. Nucleic Acids Res 35: D721-726.

118. Sabah M, Cummins R, Leader M, Kay E (2006) Aberrant expression of the Rb pathway proteins in soft tissue sarcomas. Appl Immunohistochem Mol Morphol 14: 397-403.

119. Sugimoto K, Kage H, Aki N, Sano A, Kitagawa H, et al. (2007) The induction of H3K9 methylation by PIWIL4 at the p16Ink4a locus. Biochem Biophys Res Commun 359: 497-502.

120. Brenet F, Moh M, Funk P, Feierstein E, Viale AJ, et al. (2011) DNA methylation of the first exon is tightly linked to transcriptional silencing. PLoS One 6: e14524.

121. Aravin AA, van der Heijden GW, Castaneda J, Vagin VV, Hannon GJ, et al. (2009) Cytoplasmic compartmentalization of the fetal piRNA pathway in mice. PLoS Genet 5: e1000764.

122. Grivna ST, Pyhtila B, Lin H (2006) MIWI associates with translational machinery and PIWI-interacting RNAs (piRNAs) in regulating spermatogenesis. Proc Natl Acad Sci U S A 103: 13415-13420.

123. Easwaran HP, Van Neste L, Cope L, Sen S, Mohammad HP, et al. (2010) Aberrant silencing of cancerrelated genes by $\mathrm{CpG}$ hypermethylation occurs independently of their spatial organization in the nucleus. Cancer Res 70: 8015-8024.

124. Clark SJ (2007) Action at a distance: epigenetic silencing of large chromosomal regions in carcinogenesis. Hum Mol Genet 16 Spec No 1: R88-95.

125. Assis R, Kondrashov AS (2009) Rapid repetitive element-mediated expansion of piRNA clusters in mammalian evolution. Proc Natl Acad Sci U S A 106: 7079-7082.

126. Rumbaugh G, Miller CA (2011) Epigenetic changes in the brain: measuring global histone modifications. Methods Mol Biol 670: 263-274. 
127. Beck D, Brandl MB, Boelen L, Unnikrishnan A, Pimanda JE, et al. (2012) Signal analysis for genomewide maps of histone modifications measured by ChIP-seq. Bioinformatics 28: 1062-1069.

128. Ehrlich M (2009) DNA hypomethylation in cancer cells. Epigenomics 1: 239-259.

129. Estecio MR, Issa JP (2011) Dissecting DNA hypermethylation in cancer. FEBS Lett 585: 2078-2086.

130. Siddiqi S, Terry M, Matushansky I (2012) Hiwi mediated tumorigenesis is associated with DNA hypermethylation. PLoS One 7: e33711.

131. Carey MF, Peterson CL, Smale ST (2010) Protein complex binding to promoter DNA: immobilized template assay. Cold Spring Harb Protoc 2010: pdb prot5465.

132. Kalmykova Al, Klenov MS, Gvozdev VA (2005) Argonaute protein PIWI controls mobilization of retrotransposons in the Drosophila male germline. Nucleic Acids Res 33: 2052-2059.

133. Lu J, Getz G, Miska EA, Alvarez-Saavedra E, Lamb J, et al. (2005) MicroRNA expression profiles classify human cancers. Nature 435: 834-838.

134. Duan YG, Xiong ZM, Li JX, Zhu CH, Liu ZL (2007) [Expression patterns of age-related molecule c-kit, HIWI and vimentin in the rat testis and epididymis]. Zhonghua Nan Ke Xue 13: 992-996.

135. Borel F, Konstantinova P, Jansen PL (2012) Diagnostic and therapeutic potential of miRNA signatures in patients with hepatocellular carcinoma. J Hepatol.

136. Siddiqi S, Matushansky I (2012) Piwis and piwi-interacting RNAs in the epigenetics of cancer. J Cell Biochem 113: 373-380.

137. Brower-Toland B, Findley SD, Jiang L, Liu L, Yin H, et al. (2007) Drosophila PIWI associates with chromatin and interacts directly with HP1a. Genes Dev 21: 2300-2311.

138. Deng C, Zhang P, Harper JW, Elledge SJ, Leder P (1995) Mice lacking p21CIP1/WAF1 undergo normal development, but are defective in $\mathrm{G1}$ checkpoint control. Cell 82: 675-684.

139. Baldi A, De Luca A, Esposito V, Campioni M, Spugnini EP, et al. (2011) Tumor suppressors and cellcycle proteins in lung cancer. Patholog Res Int 2011: 605042.

140. Porter PL, Malone KE, Heagerty PJ, Alexander GM, Gatti LA, et al. (1997) Expression of cell-cycle regulators p27Kip1 and cyclin $\mathrm{E}$, alone and in combination, correlate with survival in young breast cancer patients. Nat Med 3: 222-225.

141. Abou-Zeid AA, Azzam AZ, Kamel NA (2011) Methylation status of the gene promoter of cyclindependent kinase inhibitor 2A (CDKN2A) in ovarian cancer. Scand J Clin Lab Invest 71: 542-547.

142. Scott SA, Kimura T, Dong WF, Ichinohasama R, Bergen S, et al. (2004) Methylation status of cyclindependent kinase inhibitor genes within the transforming growth factor beta pathway in human T-cell lymphoblastic lymphoma/leukemia. Leuk Res 28: 1293-1301.

143. Go JH (2003) Methylation analysis of cyclin-dependent kinase inhibitor genes in primary gastrointestinal lymphomas. Mod Pathol 16: 752-755.

144. Lau NC, Seto AG, Kim J, Kuramochi-Miyagawa S, Nakano T, et al. (2006) Characterization of the piRNA complex from rat testes. Science 313: 363-367.

145. Kinney SR, Pradhan S (2011) Regulation of expression and activity of DNA (cytosine-5) methyltransferases in mammalian cells. Prog Mol Biol Transl Sci 101: 311-333.

146. Du Z, Song J, Wang Y, Zhao Y, Guda K, et al. (2010) DNMT1 stability is regulated by proteins coordinating deubiquitination and acetylation-driven ubiquitination. Sci Signal 3: ra80.

147. Szenker E, Ray-Gallet D, Almouzni G (2011) The double face of the histone variant H3.3. Cell Res 21: 421-434.

148. Santenard A, Ziegler-Birling C, Koch M, Tora L, Bannister AJ, et al. (2010) Heterochromatin formation in the mouse embryo requires critical residues of the histone variant H3.3. Nat Cell Biol 12: 853-862. 
149. Wong LH, Ren H, Williams E, McGhie J, Ahn S, et al. (2009) Histone H3.3 incorporation provides a unique and functionally essential telomeric chromatin in embryonic stem cells. Genome Res 19: 404-414.

150. Hake SB, Garcia BA, Kauer M, Baker SP, Shabanowitz J, et al. (2005) Serine 31 phosphorylation of histone variant $\mathrm{H} 3.3$ is specific to regions bordering centromeres in metaphase chromosomes. Proc Natl Acad Sci U S A 102: 6344-6349.

151. Tamura T, Smith M, Kanno T, Dasenbrock H, Nishiyama A, et al. (2009) Inducible deposition of the histone variant H3.3 in interferon-stimulated genes. J Biol Chem 284: 12217-12225.

152. Agoston AT, Argani P, Yegnasubramanian S, De Marzo AM, Ansari-Lari MA, et al. (2005) Increased protein stability causes DNA methyltransferase 1 dysregulation in breast cancer. J Biol Chem 280: 18302-18310.

153. Zhou Q, Agoston AT, Atadja P, Nelson WG, Davidson NE (2008) Inhibition of histone deacetylases promotes ubiquitin-dependent proteasomal degradation of DNA methyltransferase 1 in human breast cancer cells. Mol Cancer Res 6: 873-883.

154. Oda M, Yamagiwa A, Yamamoto S, Nakayama T, Tsumura A, et al. (2006) DNA methylation regulates long-range gene silencing of an $\mathrm{X}$-linked homeobox gene cluster in a lineage-specific manner. Genes Dev 20: 3382-3394.

155. Coolen MW, Stirzaker C, Song JZ, Statham AL, Kassir Z, et al. (2010) Consolidation of the cancer genome into domains of repressive chromatin by long-range epigenetic silencing (LRES) reduces transcriptional plasticity. Nat Cell Biol 12: 235-246.

156. Nguyen C, Liang G, Nguyen TT, Tsao-Wei D, Groshen S, et al. (2001) Susceptibility of nonpromoter CpG islands to de novo methylation in normal and neoplastic cells. J Natl Cancer Inst 93: 14651472.

157. Toguchida J, Nakayama T (2009) Molecular genetics of sarcomas: applications to diagnoses and therapy. Cancer Sci 100: 1573-1580.

158. Nyabi O, Naessens M, Haigh K, Gembarska A, Goossens S, et al. (2009) Efficient mouse transgenesis using Gateway-compatible ROSA26 locus targeting vectors and F1 hybrid ES cells. Nucleic Acids Res 37: e55.

159. Dean-Colomb W, Esteva FJ (2008) Her2-positive breast cancer: herceptin and beyond. Eur J Cancer 44: 2806-2812. 